\title{
Characterization of Aluminum Oxide Optically Stimulated Luminescent Dosimeters for Clinical Dosimetry in Radiotherapy
}

\author{
by \\ Azeez Omotayo \\ B.Sc. (University of Winnipeg)
}

A Thesis submitted to the

Faculty of Graduate and Postdoctoral Affairs

in partial fulfillment of the requirements

for the degree of

Master of Science

Ottawa-Carleton Institute for Physics

Department of Physics, Carleton University

Ottawa, Ontario, Canada

Copyright $(0$ 2012, Azeez Omotayo 
Library and Archives

Canada

Published Heritage

Branch

395 Wellington Street

Ottawa ON K1A ON4

Canada
Bibliothèque et

Archives Canada

Direction du

Patrimoine de l'édition

395 , rue Wellington

Ottawa ON K1A ON4

Canada
Your file Votre référence

ISBN: 978-0-494-93581-1

Our file Notre référence

ISBN: 978-0-494-93581-1
NOTICE:

The author has granted a nonexclusive license allowing Library and Archives Canada to reproduce, publish, archive, preserve, conserve, communicate to the public by telecommunication or on the Internet, loan, distrbute and sell theses worldwide, for commercial or noncommercial purposes, in microform, paper, electronic and/or any other formats.

The author retains copyright ownership and moral rights in this thesis. Neither the thesis nor substantial extracts from it may be printed or otherwise reproduced without the author's permission.
AVIS:

L'auteur a accordé une licence non exclusive permettant à la Bibliothèque et Archives Canada de reproduire, publier, archiver, sauvegarder, conserver, transmettre au public par télécommunication ou par l'Internet, prêter, distribuer et vendre des thèses partout dans le monde, à des fins commerciales ou autres, sur support microforme, papier, électronique et/ou autres formats.

L'auteur conserve la propriété du droit d'auteur et des droits moraux qui protege cette thèse. $\mathrm{Ni}$ la thèse ni des extraits substantiels de celle-ci ne doivent être imprimés ou autrement reproduits sans son autorisation.
In compliance with the Canadian Privacy Act some supporting forms may have been removed from this thesis.

While these forms may be included in the document page count, their removal does not represent any loss of content from the thesis.
Conformément à la loi canadienne sur la protection de la vie privée, quelques formulaires secondaires ont été enlevés de cette thèse.

Bien que ces formulaires aient inclus dans la pagination, il n'y aura aucun contenu manquant. 


\begin{abstract}
Radiation therapy requires delivery of ionizing radiation to treat cancer patients. In-vivo dosimetry is the measurement of the dose delivered during and after treatment. This work investigated the use of a commercial aluminum oxide $\left(\mathrm{Al}_{2} \mathrm{O}_{3}: \mathrm{C}\right)$ optically stimulated luminescent dosimeter (OSLD), nanoDot ${ }^{\mathrm{TM}}$ (Landauer Inc., Illinois, USA), for in-vivo dosimetry in radiotherapy.

The aim was to develop a calibration protocol that allows multiple uses of the OSLD. First, we investigated different optical treatments to reset the dosimeter after exposure to radiation. Second, we tested four different proposed calibration methods when the OSLDs were subjected to different resetting treatments.

We concluded that it is possible to re-use the nanoDot OSLDs within $\pm 3.5 \%$ accuracy up to accumulated doses of $50 \mathrm{~Gy}$, if it is used with an appropriate calibration method and optical resetting treatment. Hence, we recommend that nanoDot OSLDs can be used for clinical radiotherapy dosimetry.
\end{abstract}




\section{Statement of Originality}

Portions of this thesis are from an article published in the Medical Physics journal, in which I am the first author. The article is entitled: "Effects of different bleaching wavelengths on the absorbed-dose sensitivity of nanoDot optically stimulated luminescent dosimeters (OSLDs) exposed to 6 MV x-ray beams" Med. Phys. 39, 5457 $(2012)^{1}$. The contents from the manuscript were all from collaborations amongst Dr. Gabriel Sawakuchi (Department of Physics, Carleton University), Dr. Joanna Cygler (Department of Medical Physics, The Ottawa Hospital Cancer Center), and myself. Permission has been obtained from all collaborators and contributors, to replicate and reproduce some of the materials and contents from the publication. The contents that were replicated or reproduced from the article include texts of Sections $3.2-3.5$, Sections 4.1 - 4.4 and Sections 5.1 - 5.4. Figure 3-2, Figure 3-3, Figure 3-5, Figure 3-6, Figure 4-2, Figure 4-3, Figure 4-4, Figure 4-5, and Figure 4-6; as well as Table 4-3 and Table 4-4. 


\section{Acknowledgements}

First and foremost, I would like to express my sincere gratitude to my supervisor, Dr. Gabriel Sawakuchi, for his patience, guidance, kindness, and understanding. He has been exemplary and peerless throughout my studentship and under his supervision. He has also been a source of inspiration and motivation that science can be done with progress and achievements at a young age. I am glad he took the chance to supervise me.

I would like to specially thank my co-supervisor, Dr. Joanna Cygler, for sacrificing her evenings, and many hours in between her clinical duties and challenging profession to help me with the acquisition of data and ensure the completion of this thesis. I most appreciate your efforts.

I would also like to thank all my friends and colleagues in the Physics Department, for the time spent together and making my experience here memorable. Finally, I would like to thank my parents for giving me everything that has led to this moment. Your hopes, prayers, faith and belief were the backbone of my continued existence.

This work is dedicated to the Almighty God and my parents, Musbau and Basirat Omotayo. I thank you all and owe everything in life to you. 


\section{Table of Contents}

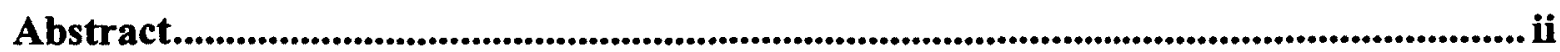

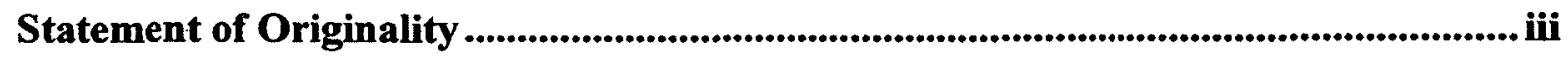

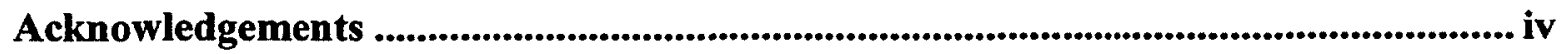

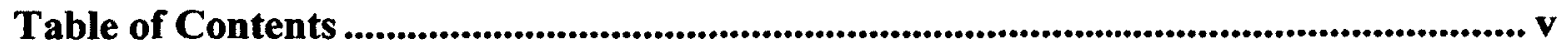

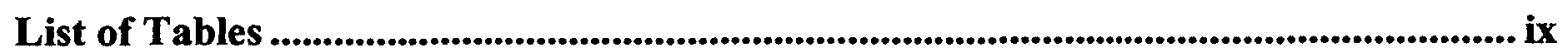

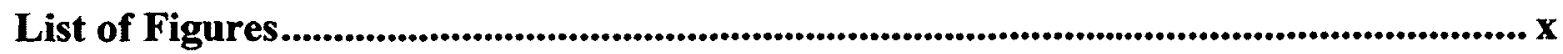

List of Acronyms and Abbreviations ................................................................... xvi

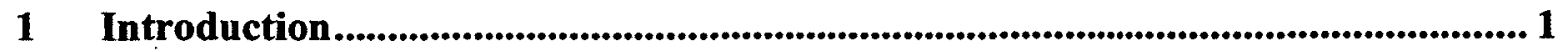

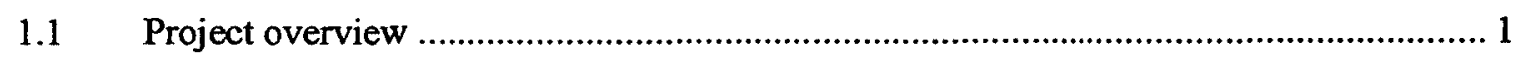

Introduction to Optically Stimulated Luminescence

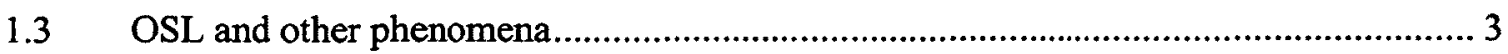

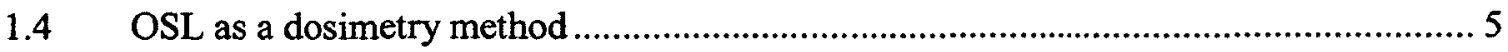

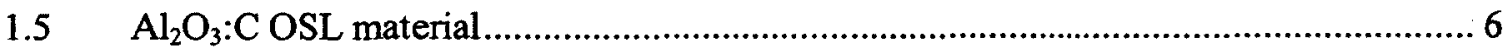

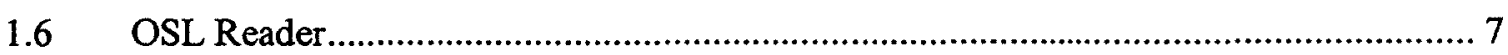

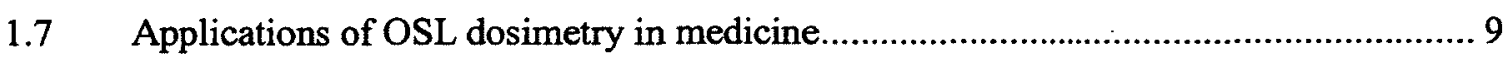

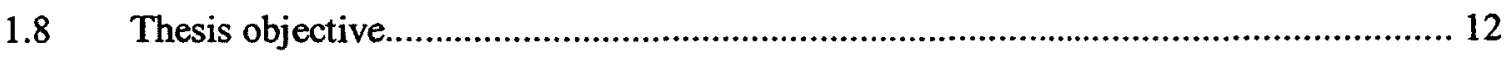

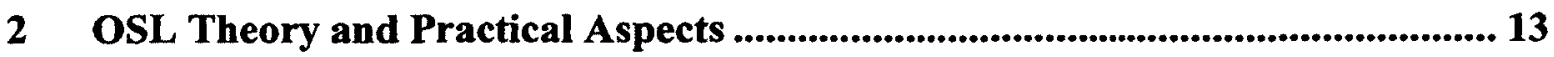

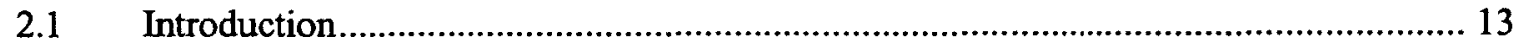

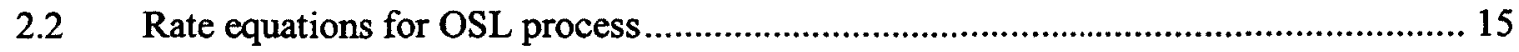

2.2.1 One Trap-One Recombination center (OTOR) model........................................... 16

2.2.2 Multiple traps and multiple center defect model .................................................. 19

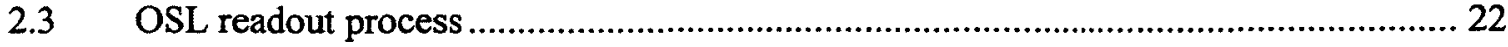

2.4 Dose measurement formalism for clinical dosimetry ............................................. 23

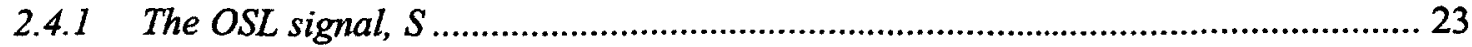




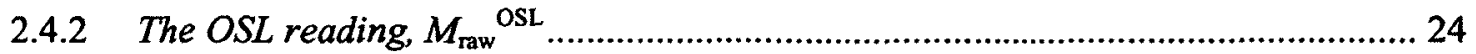

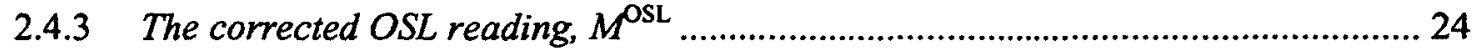

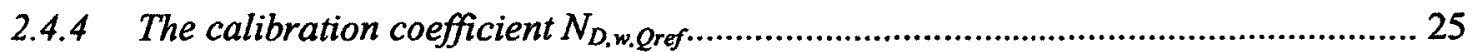

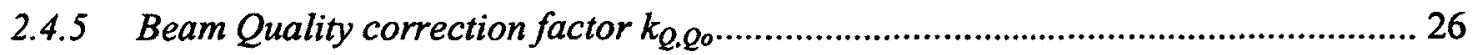

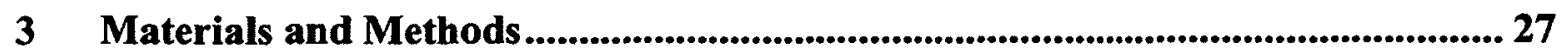

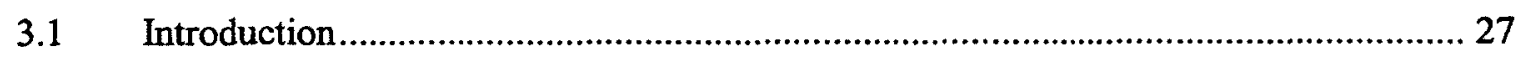

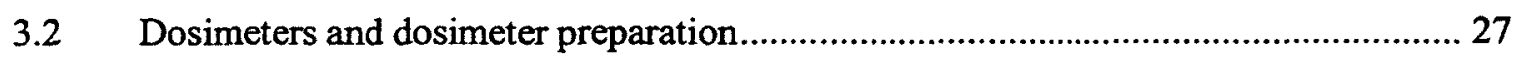

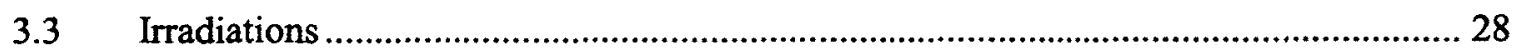

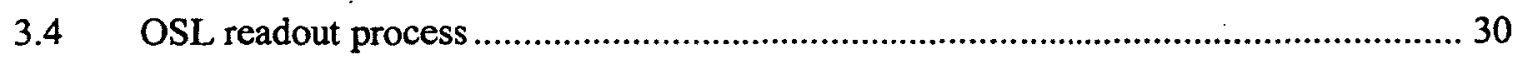

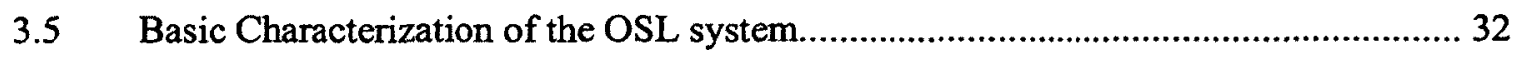

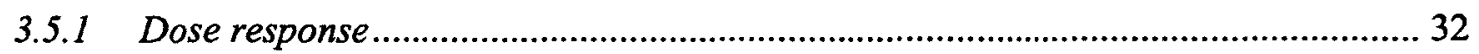

3.5.2 Loss of OSL signal as a function of sequential readouts ...................................... 33

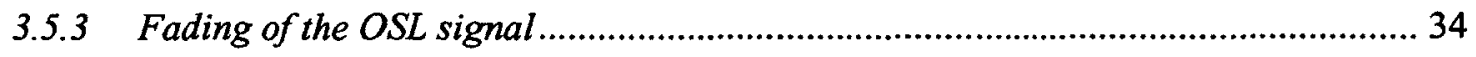

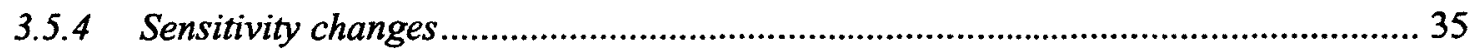

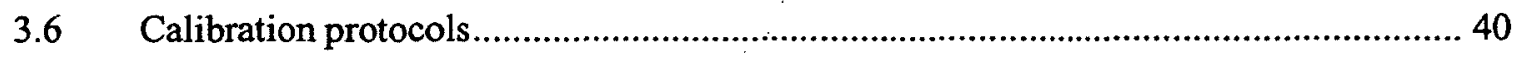

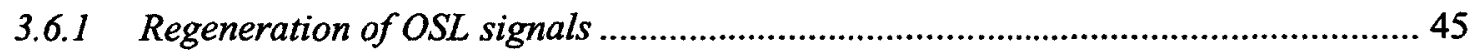

3.7 Dose determination and sensitivity change for different calibration protocols........... 45

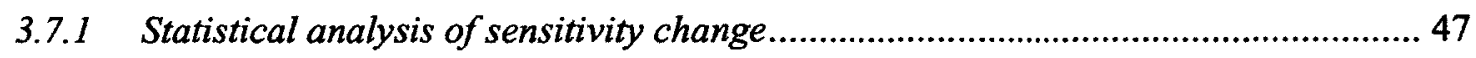

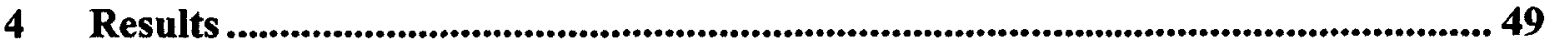

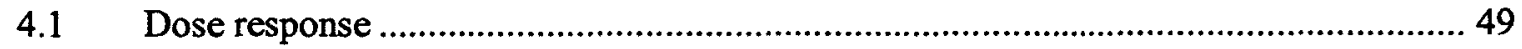

4.2 Loss of the OSL signal as a function of sequential readouts ................................... 50

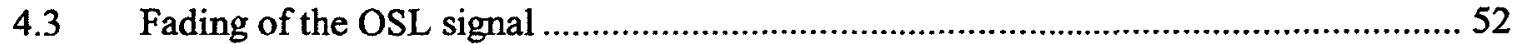

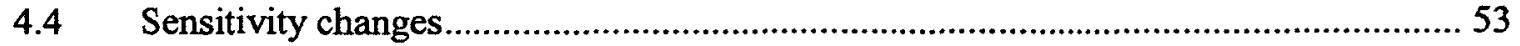

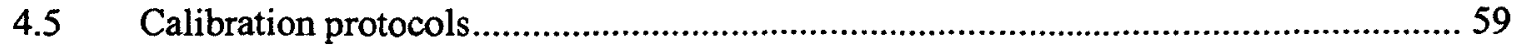

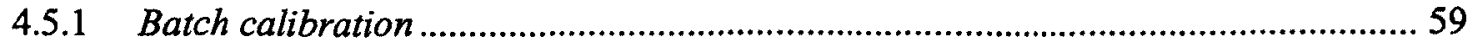

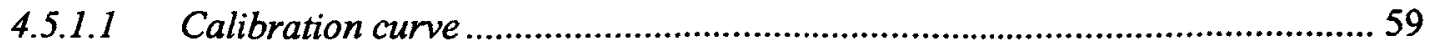




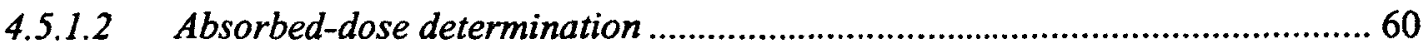

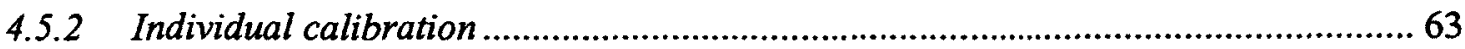

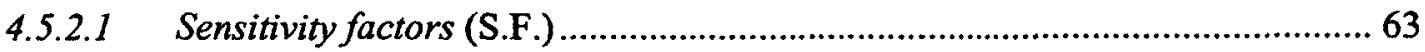

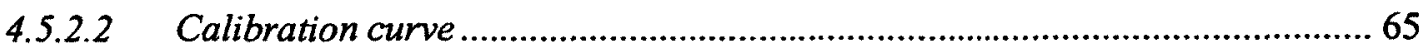

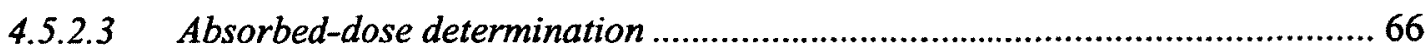

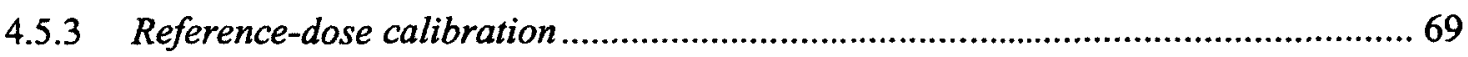

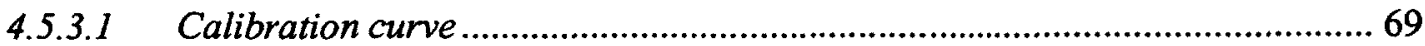

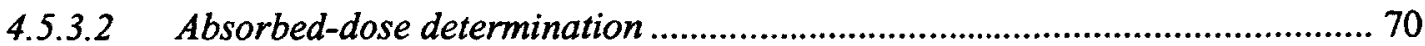

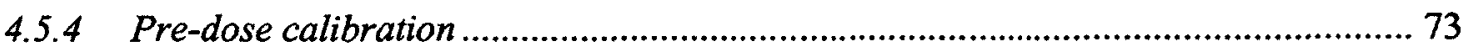

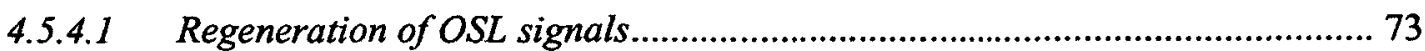

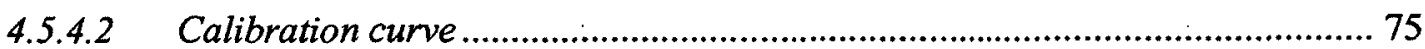

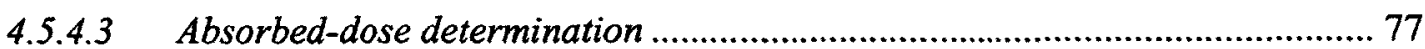

4.5.5 Comparison of absorbed-dose determination for all calibration protocols............. 82

4.6 Dose response and supralinearity per cycle for pre-dosed nanoDot OSLDs .............. 86

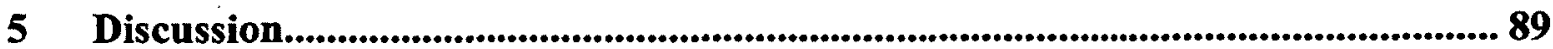

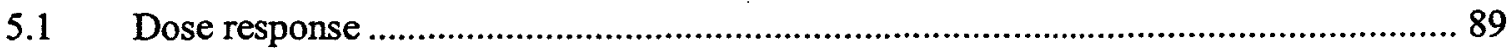

$5.2 \quad$ Loss of OSL signal as a function of sequential readouts ....................................... 89

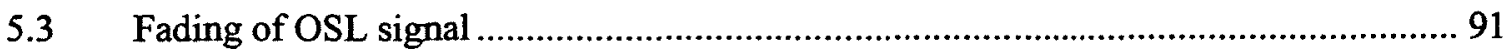

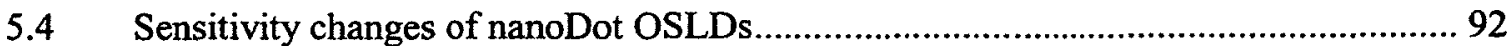

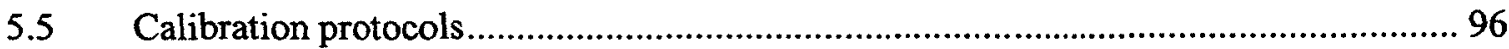

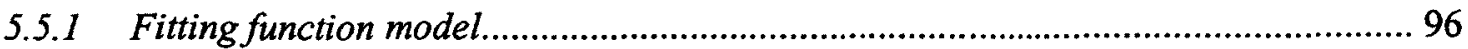

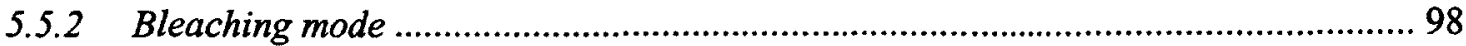

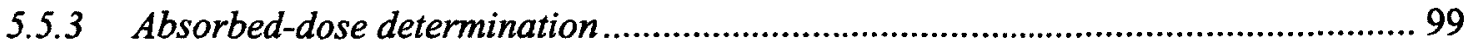

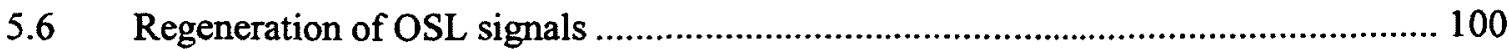

5.7 Absorbed-dose determination in OSLDs pre-exposed to $1 \mathrm{kGy} \ldots \ldots \ldots \ldots \ldots \ldots \ldots \ldots \ldots . . . . . . . . . . . . . . .101$

$5.8 \quad$ Supralinearity response per cycle 
6 Summary, Conclusions and Future Work ............................................................. 104

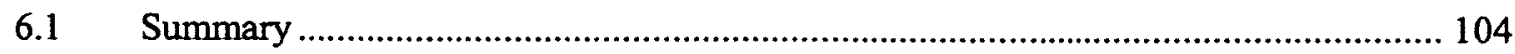

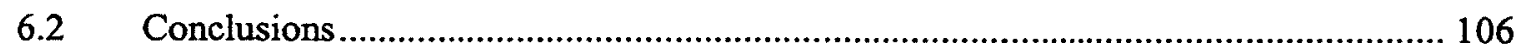

$6.3 \quad$ Future Work

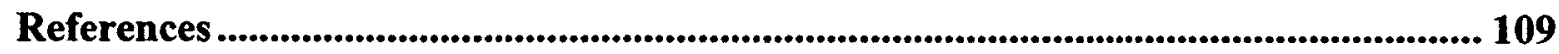




\section{List of Tables}

Table 4-1: Parameters showing the comparison of the second- and third-order fitting models of Eq. (3.5) and Eq. (3.6) for Figure 4-1 using Fisher's F-test. 50

Table 4-2: Rate of loss of OSL signal after 50 or 100 sequential readouts of nanoDot OSLDs exposed to doses from $0.1-10$ Gy as shown in Figure 4-2, following a linear fit model.

Table 4-3: Parameters determined by fitting Eq. (3.3) to the experimental data of Figure 4-3.

Table 4-4: Raw counts of the residual OSL signal after exposing the nanoDot OSLDs to 1 and $10 \mathrm{~Gy}$ and then bleaching for different periods using bleaching mode (i) (directly under the lamps) and bleaching mode (ii) (with the aid of a long-pass optical filter in front of the lamps). Except for the experiment using bleaching mode (ii) and OSLD exposed to $10 \mathrm{~Gy}$, all data were acquired for two OSLDs for each experimental condition. Values in the table are the average of three readouts for each OSLD and uncertainties are the standard deviation of the mean. Note that the readout-mode changes at 10 and $55 \mathrm{~min}$. Adapted from Omotayo et al. (2012). ${ }^{1}$

Table 4-5: Values of the $\chi^{2}$ and $\Gamma^{2}$ statistics described by Eqs. (3.9) and (3.10), comparing the two fitting models for dose estimations in Figure 4-7, for bleaching modes (i) and (ii), respectively.

Table 4-6: Sensitivity factors for 16 nanoDot OSLDs after exposure to test dose of 20 cGy. The OSLDs were bleached with mode (i) for $10 \mathrm{~min}$ before exposure to remove background signals.

Table 4-7: Sensitivity factors for 16 nanoDot OSLDs after exposure to test dose of 20 cGy. The OSLDs were bleached with mode (ii) for $30 \mathrm{~min}$ before exposure to remove background signals.

Table 4-8: Values of the $\chi^{2}$ and $\Gamma^{2}$ statistics described by Eq. (3.9) and (3.10), comparing the two fitting models for dose estimations in Figure 4-11, using bleaching mode (i) and (ii).

Table 4-9: Values of the $\chi^{2}$ and $\Gamma^{2}$ statistics described by Eq. (3.9) and (3.10), comparing the two fitting models for dose estimations in Figure 4-13, using bleaching mode (i) and (ii). 71 


\section{List of Figures}

Figure 1-1: Schematic representation of several thermally and optically stimulated phenomena. Luminescence techniques (TL and OSL) monitor the charges as they undergo radiative recombination with charges of opposite sign. Conductivity techniques (Thermally Stimulated Conductivity, TSC and Photoconductivity, PC) monitor charges after release from traps into the conduction band. Exo-electron processes: thermally stimulated exo-electron emission (TSEE), and optically stimulated exo-electron emission (OSEE) monitor the charges emitted from surface of material. Capacitance techniques (Deep Level Transient Spectroscopy, DLTS and Thermally Stimulated Capacitance, TSCap) measure signals proportional to the concentration of charge traps. Photoluminescence (PL) is however not the same type of stimulated phenomenon.......... 5

Figure 2-1: Representation of the OSL process. (a) excitation process describes period in which the dosimeter is worn by user, or exposed to an unknown radiation beam to be characterized; (b) latency process describes period in which the dosimeter is transported to laboratory and stored after exposure to radiation; (c) stimulation and readout process which gives information stored in detector for the radiation field. 15

Figure 2-2: Schematic representation of the OTOR model. The parameters are explained in the text.

Figure 2-3: A model showing the three-trap, two-center model of the $\mathrm{Al}_{2} \mathrm{O}_{3}: \mathrm{C}$ crystal (following McKeever et al. $1997^{64}$ and Bøtter-Jensen et al. $2003^{41}$ ). CB- conduction band; VB- valence band; ST- shallow trap; MDT- main dosimetry trap; DT- deep trap; RRC- radiative recombination center; and NRRC-non-radiative recombination center. 20

Figure 3-1: Landauer nanoDot OSLDs and adapter (top) used to hold the OSLDs for readout in the MicroStar reader. The plastic casing has dimensions of $10 \mathrm{~mm} \times 10 \mathrm{~mm} \times$ $2 \mathrm{~mm}$, while the sensitive volume of the dosimeter is $5 \mathrm{~mm}$ in diameter and $0.3 \mathrm{~mm}$ thick. The dosimeter on the left shows the exposed sensitive volume from the light-tight casing.

Figure 3-2: Grooved solid water phantom used for loading nanoDot OSLDs for irradiations.

Figure 3-3: Calibration and irradiation setup. Irradiations were carried out in a $6 \mathrm{MV}$ photon beam at source to surface distance (SSD) of $100 \mathrm{~cm}$ and field size $10 \mathrm{~cm} \times 10 \mathrm{~cm}$.

Figure 3-4: The InLight MicroStar reader with the open cassette for loading the nanoDot OSLDs. The reader comes with programmed software on the computer, for reading out the OSLDs. 32 
Figure 3-5: Custom-built bleaching unit containing four $26 \mathrm{~W}$ fluorescent bulbs to bleach the nanoDot OSLDs. In this picture, a long-pass filter (Schott GG-495) was placed over the nanoDot OSLDs to block wavelengths below $495 \mathrm{~nm}$. 36

Figure 3-6: Emission spectra of the custom-built bleaching unit. The solid line is the spectrum of direct illumination (bleaching mode $i$ ) and dashed line is the spectrum using a long-pass filter (Schott GG-495) between the light source and light spectrometer (bleaching mode ii).

Figure 3-7: Irradiation-readout-bleaching-readout cycle performed to assess the absorbeddose sensitivity changes of OSLDs as a function of accumulated dose.

Figure 3-8: Representation of the batch calibration method.

Figure 3-9: Representation of the individual calibration method.

Figure 3-10: Representation of the reference-dose calibration method.

Figure 4-1: OSL signal $S$ as a function of dose for nanoDot OSLDs exposed to a range of doses from 0.5 to $10 \mathrm{~Gy}$. Each data point represents the average reading of two nanoDot OSLDs where each nanoDot was read out three times. Error bars are the standard deviation of the mean. The linear behavior (represented by the blue dotted line) was determined by fitting a linear model passing through the origin in the dose range from 0.5 to $1 \mathrm{~Gy}$. Fit 1 and Fit 2 are the second and third-order polynomial models of Eq. (3.5) and Eq. (3.6), respectively.

Figure 4-2: Loss of the OSL signal after successive readouts of nanoDot OSLDs exposed to doses in the range from 0.1 to $10 \mathrm{~Gy}$. Each data point represents the OSL signal $S$ of a single readout after normalization to the OSL signal of the first readout $S_{0}$. Error bars represent the statistical fluctuations in PMT counts.

Figure 4-3: Fading of the OSL signal $S$ as a function of time elapsed since irradiation. The data sets were normalized to the last point of each respective data set $\left(S_{f}\right.$, at 2500 $2800 \mathrm{~min}$ ). Sets of two nanoDot OSLDs were exposed to doses of 1 and $10 \mathrm{~Gy}$. Each data point represents a single readout of an individual nanoDot OSLD. The error bars are the statistical fluctuation in the PMT counts. Full and dashed lines are fittings using Eq. (3.3).

Figure 4-4: OSL signal $S$ as a function of accumulated doses from repeated cycles of irradiation-readout-bleaching-readout, after relaxation of the nanoDot OSLDs in the dark for at least $10 \mathrm{~min}$. The data were normalized to the OSL signal $S_{0}$ from the first cycle. Bleaching was performed in two modes: (i) using direct illumination of the OSLDs, and (ii) using a long-pass filter in between the light source and OSLDs. Illumination was performed for: (a) 10 and $55 \mathrm{~min}, 1 \mathrm{~Gy}$; (b) 120 and $600 \mathrm{~min}, 1 \mathrm{~Gy}$; (c) 600 and $200 \mathrm{~min}$, $1 \mathrm{~Gy}$; (d) 10 and $5 \mathrm{~min}, 10 \mathrm{~Gy}$; (e) 120 and $600 \mathrm{~min}, 10 \mathrm{~Gy}$; and (f) 600 and $2000 \mathrm{~min}, 10$ Gy. Each data point represents the average of three readouts (same OSLD), with error 
bars representing the standard deviation of the mean for the three readouts. Adapted from Omotayo et al. (2012). ${ }^{1}$

Figure 4-5: Post-bleaching OSL signal $S$ as a function of accumulated doses from repeated cycles of irradiation-readout-bleaching-readout. The data were normalized to $S_{B G}=(371 \pm 48)$ counts, which is the average of the residual signal of detectors exposed to an accumulated dose of $1 \mathrm{~Gy}$ and bleached for $600 \mathrm{~min}$ and $2000 \mathrm{~min}$ using bleaching modes (i) and (ii), respectively. The caption of Figure 4-4 provides the details of this plot. Adapted from Omotayo et al. (2012). ${ }^{1}$ 57

Figure 4-6: OSL signal $S$ as a function of dose for nanoDot OSLDs exposed to doses $D$ from 0.5 to $10 \mathrm{~Gy}$. Each data point represents the average reading of three readouts of one nanoDot OSLD, except at 1 and $10 \mathrm{~Gy}$ where two OSLDs were used. Error bars are the standard deviation of the mean. The fit was determined using the model described by Eq. (3.6). 59

Figure 4-7: OSLD measured dose $D_{\text {OSL }}$ divided by the delivered dose $D$, as a function of accumulated doses for batch calibration method. Bleaching of the nanoDot OSLDs was $10 \mathrm{~h}$ for mode (i) and $40 \mathrm{~h}$ for mode (ii) for all dose fractions. Each data point represents the ratio of the OSLD measured dose $D_{O S L}$, determined by the calibration curves using fitting models from Eq. (3.7) [Fit 1] and Eq. (3.8) [Fit 2], to the dose $D$ delivered per cycle to the nanoDot OSLDs, as measured by the ion chamber. Short dashed lines represent the $\pm 5 \%$ interval, from the expected unity value (long dash). 61

Figure 4-8: Sensitivity factors for nanoDot OSLDs exposed to a test dose of $20 \mathrm{cGy}$, after bleaching with mode (i) for $10 \mathrm{~min}$ to remove background signals. S.F. values are determined by Eq. (3.4). Dashed lines represent the $5 \%$ interval from the average S.F for all the OSLDs. 64

Figure 4-9: Sensitivity factors for nanoDot OSLDs exposed to a test dose of $20 \mathrm{cGy}$, after bleaching with mode (ii) for $30 \mathrm{~min}$ to remove background signals. S.F. values are determined by Eq. (3.4). Dashed lines represent the $5 \%$ interval from the average S.F for all the OSLDs.

Figure 4-10: OSL signal $S$ divided by S.F., as a function of dose for nanoDot OSLDs exposed to doses $D$ from 0.5 to $10 \mathrm{~Gy}$. Each data point represents the average of three readouts of one nanoDot OSLD, except at 1 and 10 Gy where two OSLDs were used. Error bars are the standard deviation of the mean. The fit was determined using the model described by Eq. (3.6).

Figure 4-11: OSLD measured dose $D_{\text {OSL }}$ divided by the delivered dose $D$, as a function of accumulated doses for individual calibration method. Bleaching of the nanoDot OSLDs was $10 \mathrm{~h}$ for mode (i) and $40 \mathrm{~h}$ for mode (ii) for all dose fractions. Each data point represents the ratio of the OSLD measured dose $D_{O S L}$, determined by the calibration curves using fitting models from Eq. (3.7) [Fit 1] and Eq. (3.8) [Fit 2], to the dose $D$ 
delivered per cycle to the nanoDot OSLDs, as measured by the ion chamber. Short dashed lines represent the $\pm 5 \%$ interval, from the expected unity value (long dash)....... 68

Figure 4-12: OSL signal $S$, divided by reference-dose signal $S_{r e}$, as a function of dose for nanoDot OSLDs exposed to doses $D$ from 0.5 to $10 \mathrm{~Gy}$. $S_{\text {ref }}$ is the OSL reading after the reference dose of $20 \mathrm{cGy}$ is delivered. Each data point represents the average of three readouts of one nanoDot OSLD, except at 1 and $10 \mathrm{~Gy}$ where two OSLDs were used. Error bars are the standard deviation of the mean. The fit was determined using the model described by Eq. (3.6). 70

Figure 4-13: OSLD measured dose $D_{\text {OSL }}$ divided by the delivered dose $D$, as a function of accumulated doses for the reference-dose calibration method. Each data point represents the ratio of the OSLD measured dose $D_{O S L}$, determined by the calibration curves using fitting models from Eq. (3.7) [Fit 1] and Eq. (3.8) [Fit 2], to the dose $D$ delivered per cycle to the nanoDot OSLDs, as measured by the ion chamber. Short dashed lines represent the $\pm 5 \%$ interval, from the expected unity value (long dash). Bleaching of the nanoDot OSLDs was $10 \mathrm{~h}$ for mode (i) and $40 \mathrm{~h}$ for mode (ii) for all dose fractions...... 72

Figure 4-14: Regeneration of the OSL signal, $S$ as a function of storage duration, $t$. The OSLDs were pre-exposed to $1 \mathrm{kGy}$, and then bleached with mode (i) for $12 \mathrm{~h}$ and mode (ii) for $45 \mathrm{~h}$ to very low residual OSL signal levels equivalent to $\sim 0.5 \mathrm{cGy}$ and $\sim 0.8 \mathrm{cGy}$, respectively. Each data point represents the mean of 9 nanoDot OSLDs and error bars are the standard deviation of the mean. 74

Figure 4-15: Regeneration of the OSL signal $S$ as a function of storage time, $t$, for nanoDot OSLDs used in Figure 4-14, after re-bleaching to low residual OSL signals equivalent to $\sim 0.2 \mathrm{cGy}$ and $\sim 0.33 \mathrm{cGy}$, using bleaching mode (i) and (ii) respectively. The bleaching of the OSLDs was after regeneration of the OSL signal to the saturation level $A$ described in the text. Each data point represents the mean of 9 nanoDot OSLDs and error bars are the standard deviation of the mean. 75

Figure 4-16: OSL signal $S$ as a function of dose for OSLDs exposed to pre-dose of $1 \mathrm{kGy}$, bleached to low residual signal, and irradiated with doses $D$ from 0.5 to $10 \mathrm{~Gy}$. Each data point represents the average of three readouts of one nanoDot OSLD, except at 1 and 10 Gy where two OSLDs were used. Error bars are the standard deviation of the mean. The fit was determined using the model described by Eq. (3.6). 76

Figure 4-17: OSL signal $S$, divided by S.F., as a function of dose for nanoDot OSLDs exposed to pre-dose of $1 \mathrm{kGy}$, bleached and irradiated with doses $D$ from 0.5 to $10 \mathrm{~Gy}$. Data points are as described in Figure 4-16. 76

Figure 4-18: OSL signal $S$, divided by reference OSL signal $S_{r e f}$, as a function of dose for nanoDot OSLDs exposed to a pre-dose of $1 \mathrm{kGy}$, bleached to low residual signals and exposed to doses $D$ from 0.5 to $10 \mathrm{~Gy}$. Data points are as described in Figure 4-16....... 77 
Figure 4-19: OSLD measured dose $D_{\text {OSL }}$ divided by the delivered dose $D$, as a function of cycle for pre-dose calibration method using the individual calibration definition. Each data point represents the ratio of the OSLD measured dose $D_{O S L}$, determined by the calibration curves using fitting models from Eq. (3.7) [Fit 1] and Eq. (3.8) [Fit 2], to the dose $D$ delivered per cycle to the nanoDot OSLDs, as measured by the ion chamber. Dashed lines represent the expected unity value. Bleaching of the nanoDot OSLDs was $10 \mathrm{~h}$ for mode (i) and $40 \mathrm{~h}$ for mode (ii) for all dose fractions. 79

Figure 4-20: OSLD measured dose $D_{\text {OSL }}$ divided by the delivered dose $D$, as a function of cycles for the pre-dose calibration method using the individual calibration definition. Each data point represents the ratio of the OSLD measured dose $D_{o s L}$, determined by the calibration curves using fitting models from Eq. (3.7) [Fit 1] and Eq. (3.8) [Fit 2], to the dose $D$ delivered per cycle to the nanoDot OSLDs, as measured by the ion chamber. Dashed lines represent the expected unity value. Bleaching of the nanoDot OSLDs was $10 \mathrm{~h}$ for mode (i) and $40 \mathrm{~h}$ for mode (ii) for all dose fractions. 80

Figure 4-21: OSLD measured dose $D_{\text {OSL }}$ divided by the delivered dose $D$, as a function of cycles for the pre-dose calibration method using the reference-dose calibration definition. Each data point represents the ratio of the OSLD measured dose DosL, determined by the calibration curves using fitting models from Eq. (3.7) [Fit 1] and Eq. (3.8) [Fit 2], to the dose $D$ delivered per cycle to the nanoDot OSLDs, as measured by the ion chamber. Dashed lines represent the expected unity value. Bleaching of the nanoDot OSLDs was $10 \mathrm{~h}$ for mode (i) and $40 \mathrm{~h}$ for mode (ii) for all dose fractions. 81

Figure 4-22: OSLD measured dose $D_{\text {OSL }}$ divided by the delivered dose $D$, as a function of accumulated doses for all calibration methods. Each data point represents the ratio of the OSLD measured dose $D_{O S L}$, determined by the calibration curves using fitting model from Eq. (3.8) [Fit 2], to the dose $D$ delivered per cycle to the nanoDot OSLDs, as measured by the ion chamber. Dashed lines represent the $\pm 5 \%$ interval, from the expected unity value. Bleaching of the nanoDot OSLDs was $10 \mathrm{~h}$ using mode (i) for all dose fractions. 84

Figure 4-23: OSLD measured dose $D_{\text {OsL }}$ divided by the delivered dose $D$, as a function of accumulated doses for all calibration methods. Each data point represents the ratio of the OSLD measured dose $D_{\text {OSL }}$, determined by the calibration curves using fitting model from Eq. (3.8) [Fit 2], to the dose $D$ delivered per cycle to the nanoDot OSLDs, as measured by the ion chamber. Dashed lines represent the $\pm 5 \%$ interval, from the expected unity value. Bleaching of the nanoDot OSLDs was $40 \mathrm{~h}$ using mode (ii) for all dose fractions. 85

Figure 4-24: OSL signal $S$ as a function of dose per irradiation-readout-bleaching-readout cycle, for OSLDs pre-exposed to $1 \mathrm{kGy}$, bleached to low residual signals, and irradiated with doses $D$ from 0.5 to $10 \mathrm{~Gy}$. The black solid line represents the fit to the OSL signal after bleaching with mode (i), while the red dashed line is the fit to the signal after bleaching with mode (ii). The fits were determined using the model described by Eq. (3.6). 87 
Figure 4-25: Supralinearity plots per irradiation-readout-bleaching cycle for nanoDot OSLDs exposed to $1 \mathrm{kGy}$ pre-dose, using both bleaching modes (i) and (ii). Each data point shows the supralinearity index described by Eq. (3.3). 


\section{List of Acronyms and Abbreviations}
AAPM
American Association of Physicists in Medicine
CB
Conduction Band
CW-OSL
Continuous Wave-Optically Stimulated Luminescence
DT
Deep Trap

LED

Light Emitting Diode

LM-OSL

Linear Modulation-Optically Stimulated Luminescence

MDT

Main Dosimetry Trap

MOSFET

Metal-Oxide Semiconductor Field Effect Transistor

MU

Monitor Unit

NRRC

Non-Radiative Recombination Center

OSL

Optically Stimulated Luminescence

OSLDs

Optically Stimulated Luminescent Dosimeters

PMT

Photomultiplier Tube

POSL

Pulsed Optically Stimulated Luminescence

RRC

Radiative Recombination Center

ST

Shallow Trap

TG

Task Group

TL

Thermoluminescence

TLDs

Thermoluminescent Dosimeters

QA

Quality Assurance

VB

Valence Band 


\section{Introduction}

\subsection{Project overview}

Clinical radiotherapy treatments use ionizing radiation including $\mathrm{x}$ rays, gamma rays, electrons, protons and heavy charged particles to treat cancer patients. These treatments require accurate delivery of radiation to a particular region of interest in a patient to kill cancerous cells or tumors. A high accuracy in absorbed-dose (energy imparted per unit mass) determination and delivery to the target volume is paramount to guarantee the quality of procedures while minimizing the dose to healthy tissue. ${ }^{2,3}$

Dosimeters have been used to perform quality assurance (QA) checks to monitor the accuracy and determination of doses for diagnostic and therapy purposes. In-vivo dosimetry consists of measuring the dose delivered to an individual patient using detectors placed on the patient's skin to measure entrance or exit doses. ${ }^{4}$ It has also been recommended as part of the QA program to prevent treatment errors such as set-up errors. ${ }^{4-6}$ In-vivo dosimetry requires the use of small and simple dosimeters with minimal uncertainties to obtain accuracy in absorbed-dose delivery of $3-4 \% .{ }^{4,7,8}$ Different types of dosimeters have been used to perform in-vivo dose measurements in the clinic including silicon diodes, ${ }^{4,9-11}$ MOSFET detectors, ${ }^{12,13}$ radio-chromic films, ${ }^{14,15}$ radiographic films, ${ }^{16,17}$ and thermoluminescent dosimeters (TLDs). ${ }^{9,18,19}$ Some of the mentioned dosimeters have also been investigated for other medical dosimetry applications including postal dose audit in radiation therapy centers, ${ }^{3,20,21}$ dose verification in radiation therapy, ${ }^{12,22-24}$ diagnostic imaging, ${ }^{25-27}$ and validation of MonteCarlo dose calculations. ${ }^{28}$ 
TLDs and silicon diodes have been used widely for in-vivo dosimetry in the clinic for over 50 years. ${ }^{4,29}$ Recently, a material that exhibits both thermally and optically stimulated luminescence (OSL) has been developed. ${ }^{30}$ It is made from sapphire $\left(\mathrm{Al}_{2} \mathrm{O}_{3}: \mathrm{C}\right)$ with characteristics that make it a good in-vivo dosimeter ${ }^{31}$ including 30-60 times higher sensitivity to radiation compared to TLDs, ${ }^{32}$ low levels of signal fading, ${ }^{33}$ and easily reproducible readout process. This material has been investigated for various radiation oncology applications. $^{23,27,33-37}$

The use of TLDs and other in-vivo dosimetry systems has been recommended ${ }^{4,38}$ and a protocol to use TLDs has been established. ${ }^{6,11}$ However, there is no established protocol for the use of OSL dosimeters (OSLDs) in the radiotherapy clinic. A task group of the American Association of Physicists in Medicine (AAPM TG-191), with the mandate and responsibility to develop a generally acceptable protocol for OSLD use in clinical dosimetry has been formed, with recommendations still pending. These efforts are driven by the goal of minimizing the uncertainties associated with dose delivered in radiotherapy and the need for consistent dosimetry in clinical trials and epidemiological studies. ${ }^{18}$

The objectives of this research are to characterize the properties and response of $\mathrm{Al}_{2} \mathrm{O}_{3}: \mathrm{C}$ OSLDs to different influence factors and to investigate different calibration protocols for multiple-use of the OSLDs in a clinical radiotherapy dosimetry protocol. The results of this study are expected to help in developing an acceptable calibration protocol for use of OSLDs in the clinic, aligning with the recommendations of AAPM TG-40 and implementation in the ongoing AAPM TG-191 report. 


\subsection{Introduction to Optically Stimulated Luminescence}

Sir Robert Boyle first observed luminescence in 1663 during an experiment using a diamond sample. He observed a glow (self-shining light) after heating the diamond piece. The observed phenomenon was later to be called thermoluminescence (TL) or more accurately Thermally Stimulated Luminescence. 39,40

TL refers to the process of stimulating, using thermal energy, the emission of luminescence from a substance following the absorption of energy from an external source by that substance. ${ }^{39}$ The source of external energy is usually ionizing radiation. This makes TL closely related to phosphorescence, which is the 'after-glow' light emitted by a material after absorption of external energy.

OSL is a related phenomenon whereby a material emits light after being stimulated by optical energy. It is also known as photo-stimulated luminescence (PSL). OSL differs from photoluminescence because there is no prompt emission (or fluorescence) during the absorption of the stimulation energy. ${ }^{41}$ OSL and TL are also different from photoluminescence because the latter does not necessarily involve prior absorption of energy from an external ionizing source. Photoluminescence requires the emitted light to have a longer wavelength than the stimulation light, which is not necessarily the case with OSL. Furthermore, the lifetime of photoluminescence emission decays promptly after ceasing stimulation. ${ }^{39}$

\subsection{OSL and other phenomena}

The knowledge of defect and band structures in crystals or semiconductor materials led to a better understanding of the stimulated luminescence phenomena. OSL as explained 
earlier occurs during exposure to light. The process begins with irradiation causing excitation of valence electrons. The energy is stored in the material due to localized preexisting defects, which trap the charge carriers (now electrons and holes created due to ionized electrons). Subsequently, the illumination of the irradiated material leads to absorption of energy by the trapped electrons, which transition into the delocalized conduction band. The recombination of the freed electrons with the localized holes results in radiative emission and luminescence.

The emission decays with a time constant dependent on the wavelength and intensity of the stimulation light and characteristics of the defects. In crude terms, the OSL intensity is a function of the absorbed dose by the material. OSL signals are often accompanied by photoconductivity. OSL is one of a class of stimulated phenomena. Such phenomena may be thermally stimulated (TSP) or optically stimulated (OSP). TSP include TL, thermally stimulated conductivity (TSC), thermally stimulated exo-electron emission (TSEE), thermally stimulated capacitance (TSCap) and deep level transient spectroscopy (DLTS). Likewise, OSP includes OSL, photoconductivity (PC) and optically stimulated exo-electron emission (OSEE) ${ }^{39,41}$ The relationship between these different phenomena is illustrated in Figure 1-1 using a schematic energy band diagram, following the works and treatment by Chen and McKeever, $1997^{40}$ and Bøtter-Jensen et al., $2003 .^{41}$ 


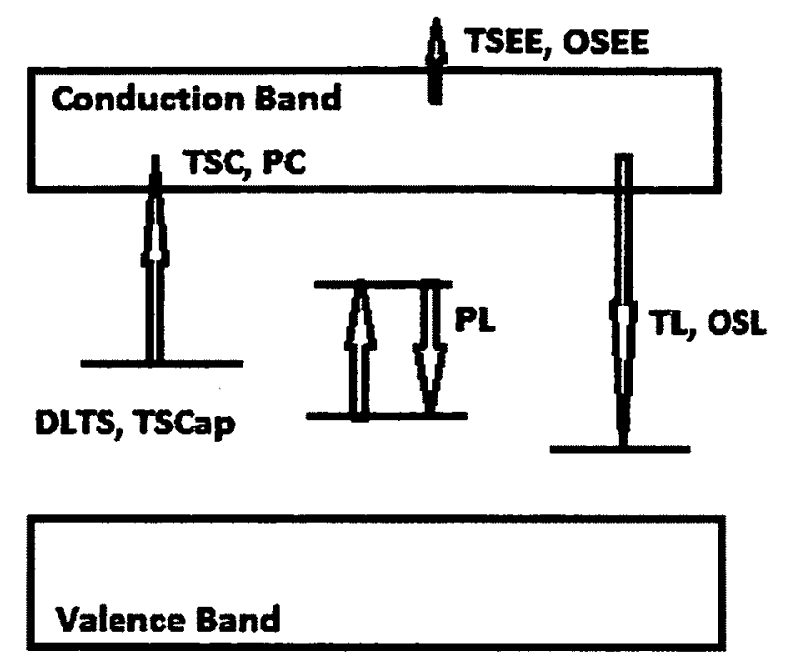

Figure 1-1: Schematic representation of several thermally and optically stimulated phenomena.

Luminescence techniques (TL and OSL) monitor the charges as they undergo radiative recombination with charges of opposite sign. Conductivity techniques (Thermally Stimulated Conductivity, TSC and Photoconductivity, PC) monitor charges after release from traps into the conduction band. Exo-electron processes: thermally stimulated exo-electron emission (TSEE), and optically stimulated exo-electron emission (OSEE) monitor the charges emitted from surface of material. Capacitance techniques (Deep Level Transient Spectroscopy, DLTS and Thermally Stimulated Capacitance, TSCap) measure signals proportional to the concentration of charge traps. Photoluminescence (PL) is however not the same type of stimulated phenomenon.

\subsection{OSL as a dosimetry method}

The OSL phenomenon as described above makes it a viable method for dosimetry, as the intensity emitted is a function of the absorbed dose from radiation. The first OSL measurements were made by Antonov-Romanovskii et al., ${ }^{42}$ monitoring the intensity of infrared stimulated luminescence from various phosphors after irradiation. Bräunlich et $a l{ }^{43}$ and Sanborn and Beard ${ }^{44}$ later carried out similar studies on sulfides. ${ }^{41}$

However, the use of sulfide materials as a viable OSL dosimeter still had limitations due to the effect of the loss of the dosimetric signal in the material at room temperature (fading). The ability of infrared light to stimulate the trapped electrons showed that the localized traps were shallow and thus required small detrapping or 
activation energy. Hence, the trapped electrons were unstable at room temperature and decayed through thermal stimulation and subsequent phosphorescence.

A major breakthrough for use of OSL in dosimetry occurred in archaeological and geological dating. Archaeological samples (natural quartz and/or feldspar) were exposed by Huntley $e t$ al $^{45}$ to a steady source of light (argon ion laser) of appropriate wavelength and intensity, and the luminescence stimulated was used to estimate the absorbed-dose received by this mineral in nature. Through an estimation of the environmental dose rate (from natural quantities of uranium), the age of the mineral sample could be determined.

\section{5 $\quad \mathrm{Al}_{2} \mathrm{O}_{3}: \mathrm{C}$ OSL material}

OSL has been observed in a variety of materials, both natural and artificial. Natural materials where OSL has been observed include quartz, feldspar, topaz, dental enamel, porcelain and ceramics. Artificial materials known to exhibit OSL include carbon-doped aluminum oxide $\left(\mathrm{Al}_{2} \mathrm{O}_{3}: \mathrm{C}\right)$, beryllium oxide $(\mathrm{BeO})$, halides $\left(\mathrm{KCl}, \mathrm{KBr}, \mathrm{NaCl}, \mathrm{CaF}_{2}\right)$ and sulphides. ${ }^{39,41,46,47}$

The most common OSL material currently used in dosimetry is carbon-doped aluminum oxide, $\mathrm{Al}_{2} \mathrm{O}_{3}: \mathrm{C}$. It was earlier used in $\mathrm{TL}$ dosimetry under the commercial name TLD-500. It was originally developed by Urals Polytechnical Institute in Russia. ${ }^{48}$ The TL of $\mathrm{Al}_{2} \mathrm{O}_{3}: \mathrm{C}$ was reported to possess a sensitivity 40-60 times that of TLD$100,{ }^{32,49}$ a common TL material consisting of lithium fluoride. $\mathrm{Al}_{2} \mathrm{O}_{3}: \mathrm{C}$ crystals are grown in a reducing atmosphere in the presence of carbon impurities, which act as catalysts to produce oxygen vacancy centers. These oxygen vacancy centers are responsible for the main OSL emission band centered at around $415 \mathrm{~nm} \cdot{ }^{30,50,51} \mathrm{Al}_{2} \mathrm{O}_{3}: \mathrm{C}$ 
OSL dosimeters are produced by Landauer Inc. (Glenwood, IL USA) under the commercial names Luxel ${ }^{\mathrm{TM}}$ and InLight ${ }^{\mathrm{TM}}$ dosimeters for personnel dosimetry; and nanoDot ${ }^{\mathrm{TM}}$ for clinical dosimetry. The sensitive part of these dosimeters is composed of $\mathrm{Al}_{2} \mathrm{O}_{3}: \mathrm{C}$ powder attached to a plastic substrate and rolled into long strips. The strips are then cut into different sizes to produce the commercial forms of the dosimeter.

The Luxel OSLDs are cut into discs of $7 \mathrm{~mm}$ diameter and $0.3 \mathrm{~mm}$ thickness and the nanoDot OSLDs cut into $5 \mathrm{~mm}$ diameter and $0.3 \mathrm{~mm}$ thickness. These dosimeters are then read in commercial readout systems for different dosimetry purposes including personnel, environmental, space and clinical.

\subsection{OSL Reader}

The essential elements required to read an OSL signal are:

(i) a light source to stimulate the trapped charges from the OSLD;

(ii) a light transducer to detect luminescence from the OSLD. Usually the light transducer is a photomultiplier tube (PMT);

(iii) a stimulation filter placed between the light source and the OSLD to select the stimulation wavelength;

(iv) a detection filter placed between the PMT and the OSLD, to block the scattered stimulation light and isolate specific OSL emission bands characteristic of the OSL material;

(v) a light tight enclosure to secure all the materials and avoid exposure of the OSLD to external light. 
The main problem in designing an OSL reader is discriminating the OSL light from the stimulation light. Therefore, there is usually a signal-to-noise effect that needs to be accounted for in optimizing the reader's detection of the OSL signal. ${ }^{39}$ Different OSL readers have been developed based on the readout modes employed in reading the detectors. The most common commercially available readout systems are the Risø TL/OSL reader (Risø National Laboratory, Denmark) and the MicroStar reader (Landauer Inc., USA).

The Risø reader is an automated reader capable of carrying out sequences of OSL and TL measurements including the irradiations and heating of up to 48 samples. Irradiations can be performed with a pre-installed radiation source. It uses light emitting diode (LED) clusters (blue, green or infrared) for the OSL stimulation, and the OSL is detected and measured by a PMT. A changeable long-pass filter is used in front of the LED to isolate short wavelengths that may reach the PMT. The reader contains a heating element that provides the ability to perform OSL/TL measurements at high temperatures. However, it should be stated that this reader is mostly used for research purposes as it has capabilities to perform different measurements such as ability to provide pulsed and linear modulated stimulations.

The MicroStar reader is a commercial readout system that uses sets of LEDs (green, $525 \mathrm{~nm}$ ) for OSL stimulation in two modes: "weak-" and "strong-" stimulation modes. The weak-stimulation mode uses 6 LEDs to produce low stimulation intensity for readout of the detectors irradiated with doses higher than approximately $0.1 \mathrm{~Gy}$, while the strong-stimulation mode uses 38 LEDs to produce high stimulation intensity for readout of the detectors irradiated with doses less than approximately $0.1 \mathrm{~Gy}$. 


\subsection{Applications of OSL dosimetry in medicine}

Recalling from Section 1.4, the acceptance of OSL as a dosimetry method was as a result of its application as a dosimeter in geological dating of mineral sediments. ${ }^{45}$ However, OSL has found its use in other dosimetry applications, including personnel, environmental, space, medical and retrospective and/or accident dosimetry. ${ }^{35,39,41,52,53} \mathrm{~A}$ brief description of the applications of OSL in medical dosimetry can be seen below. Detailed descriptions of the other mentioned OSL applications can be found in Yukihara and McKeever, $2010 .^{39}$

The main purpose of a dosimeter in medical dosimetry is to estimate the absorbed-dose delivered at a specific point in a medium of interest, typically in a water phantom or tissue, in the absence of the dosimeter. In ideal situations, dosimeters made of the same material as the tissue or water is required to avoid disturbance of the radiation beam. However, most dosimeters do not have the same chemical composition as water or tissue, making the response dependent on the energy of the beam. An ideal dosimeter should have no radiation beam dependence and also produce a signal that is linearly proportional to the absorbed-dose for a given field. ${ }^{39}$

Medical dosimetry requires very small dosimeters capable of being used in-vivo during radiotherapy treatments. The high sensitivity of the OSLDs makes viable the use of small sized dosimeters, to produce a high spatial resolution. ${ }^{54}$ The all-optical nature of the OSL process make possible the use of these dosimeters with optical fibers to measure dose in difficult to access locations, inside the human body. ${ }^{55-59}$

The use of $\mathrm{Al}_{2} \mathrm{O}_{3}: \mathrm{C}$ OSLDs for passive and in-vivo dosimetry for radiotherapy clinical applications are becoming more recently available. However, there is not a 
widely established protocol formulated for its use for clinical dosimetry. Yukihara et al., $2005^{49}$ investigated the use of commercial $\mathrm{Al}_{2} \mathrm{O}_{3}: \mathrm{C}$ OSLDs (Luxel type) for passive dosimetry using photon (6 and $18 \mathrm{MV}$ ) and electron beams $(6-20 \mathrm{MeV})$. They investigated the dose response; depth dose curves; the effect of dose rate, field size and irradiation temperature; and the energy dependence on the OSL response. They found that the dose rate, field size, and temperature changes did not change the OSL response by more than $\pm 1 \%$. It was also reported that the beam energy dependence for photon beams at $6 \mathrm{MV}$ and $18 \mathrm{MV}$, had only a $(0.51 \pm 0.48) \%$ difference in response, while a $1.9 \%$ difference was seen for electrons when compared to photons. ${ }^{49}$ However, Schembri and Heijmen $2007^{34}$ reported a difference of $4.1 \%$ between 6 and $18 \mathrm{MV}$ photon beams, while reporting a difference of $3.7 \%$ between photon and electron beams irradiated in a polystyrene phantom. Jursinic $2007^{24}$ presented a detailed study characterizing the response of a similar commercial $\mathrm{Al}_{2} \mathrm{O}_{3}: \mathrm{C}$ OSLD (InLight Dot type) to photons ( 6 and 15 MV), electrons (6-20 MeV) and Ir-192 gamma rays. He reported no dependence on the energy response for photons and electrons, a $6 \%$ higher sensitivity for Ir-192, no sensitivity to orientation and temperature of irradiation, no sensitivity dependence on dose-per-pulse, and a linear dose response up to $3 \mathrm{~Gy}$, with small supralinearity afterwards.

Similarly, Viamonte et al., $2008^{22}$ investigated the dosimetric characteristics of the same OSLD (Dot type) to photons (6 - 18 MV) and gamma rays (Cobalt-60), reporting no energy dependence for photons, but a $4 \%$ lower response relative to Co- 60 gamma rays. Reft $2009^{36}$ studied the energy response and dose response of the same $\mathrm{Al}_{2} \mathrm{O}_{3}: \mathrm{C}$ OSLD (Dot type) to photons $(125 \mathrm{kVp}-18 \mathrm{MV}$ ) and electrons $(6-10 \mathrm{MeV})$. 
He reported a linear response until 2 Gy with supralinearity afterwards; no dependence on energy for megavoltage photons and electrons; a $4.5 \%$ higher response for Co-60 and an increasing response with decreasing energy for $\mathrm{kVp}$ photons, relative to $6 \mathrm{MV}$ photons. ${ }^{36}$

$\mathrm{Al}_{2} \mathrm{O}_{3}: \mathrm{C}$ OSLDs can be effectively reset by annealing the dosimeter to $900^{\circ} \mathrm{C}$ for 15 min. ${ }^{51}$ This thermal treatment is sufficient to effectively empty all the electron traps in the lattice defects created by irradiation, and re-establish the initial un-irradiated conditions of the material. However, commercial types of $\mathrm{Al}_{2} \mathrm{O}_{3}: \mathrm{C}$ OSLDs are made of $\mathrm{Al}_{2} \mathrm{O}_{3}: \mathrm{C}$ powder fixed on a transparent plastic slide. Because of the presence of the plastic, it is not possible to apply a thermal treatment to the OSLDs, thus, the alternative is to use light to empty or "bleach" some of the electron traps. However, emptying the electron traps with illumination is not as effective as annealing. The inability to completely empty all the traps causes changes in the sensitivity of the OSLD as a function of accumulated dose. Hence, to re-use the dosimeters multiple times in a multiple-use protocol in order to minimize costs, it would be important to determine ways to minimize and account for the changes in sensitivity of the dosimeters.

Investigations have also been done to determine suitable calibration protocols for the use of $\mathrm{Al}_{2} \mathrm{O}_{3}: \mathrm{C}$ OSLDs for radiotherapy clinical dosimetry. Viamonte et al., $2008^{22}$ investigated commercial OSLDs, using $\mathrm{Al}_{2} \mathrm{O}_{3}: \mathrm{C}$ InLight 'Dot' dosimeters in a batch of 165 detectors. They reported a batch uniformity of approximately $4 \%$ (1 standard deviation) in the 'Dot' dosimeters exposed to the same irradiation conditions using a single calibration factor for all the detectors in the batch. Jursinic $2007^{24}$ reported the stability of the sensitivity of similar 'Dot' dosimeters used repeatedly to be $0.63 \%$. 
Mrčela et al., $2011^{33}$ reported a $2.5 \%$ sensitivity increase in dose response with a reproducibility of $1 \%$. Yukihara et al., $2005^{60}$ proposed a calibration protocol for $\mathrm{Al}_{2} \mathrm{O}_{3}: \mathrm{C}$ OSLDs taking into account the changes in the sensitivities of individual dosimeters, obtaining a precision of approximately $0.7 \%$.

This thesis investigates different calibration protocols based on these earlier studies to determine the suitable best calibration protocol for multiple-use in radiotherapy applications.

\subsection{Thesis objective}

Several studies have recommended that the accuracy in absorbed-dose delivery to the tumor volume should be of $3-4 \%$ for local tumor control ${ }^{4,38}$ in daily radiotherapy applications. It has also been recommended that a deviation of more than $\pm 5 \%$ in delivered dose ${ }^{7,61,62}$ should be investigated with diode or TLD dosimetry. ${ }^{4}$ However, uncertainties in the resulting dose at a point as small as 1-2\% (1 SD) can be obtained if proper calibration and correction procedures of the dosimetry system are available., ${ }^{438}$

Hence, the main objective of this thesis is to characterize the response of nanoDot OSLDs to $6 \mathrm{MV}$ radiotherapy photon beams, aiming to develop a suitable calibration protocol for the use of the nanoDot OSLDs for radiotherapy applications in a clinical workflow. The goal is to achieve accuracy in absorbed-dose measurements within $5 \%$ when re-using the nanoDot OSLDs. The major significance and relevance of this work is to provide data for the ongoing AAPM TG-191 Report. 


\section{OSL Theory and Practical Aspects}

\subsection{Introduction}

It can be recalled from the previous chapter that the energy absorbed from an ionizing radiation source causes the excitation of free electrons and free holes, which are subsequently trapped at localized defects in the band gap of the material. Optical stimulation leads to the liberation of charge carriers of one sign (usually electrons), which then recombines with charge carriers of opposite sign.

The radiation absorption leads to perturbation of the system from thermodynamic equilibrium to a metastable state. Subsequently, the absorption of external energy by the metastable state results in stimulated relaxation of the system back to the equilibrium state. During the relaxation process, recombination of electronic charge occurs, which if radiative, results in luminescence emission. ${ }^{41}$ In the case of OSL, the stimulating energy source is light (ultraviolet, visible or infra-red).

In OSL, the intensity of emitted luminescence is related to the rate at which the system returns to equilibrium. This rate is a function of the concentration of trapped metastable charges, which is linearly proportional to the trapped charge concentration in the simplest case. The integral of the luminescence intensity-versus-time curve is related to the trapped charges concentration, which in turn is proportional to the initial dose of ionizing radiation. ${ }^{41}$ This is the basis of using OSL in radiation detection and measurement.

An illustration of the process described above can be seen in Figure 2-1. In the first stage, the OSLD is exposed to a source of ionizing radiation, and the energy deposited by the radiation results in excitations of valence electrons, which are promoted 
to the conduction band, leaving behind holes in the valence band. The free electrons and holes may become trapped in defects inherent in the crystal lattice, whose energy levels are represented by the short horizontal lines in the band gap (see Figure 2-1).

The state has now been perturbed from equilibrium to a metastable state, which is a latency period characterized by a certain concentration of trapped electrons and holes. If the trapping center possesses deep potential wells, there would be a negligible probability of the trapped charges escaping due to thermal induction at room temperature. The probability of escape if the potential wells were shallow or deep, is dependent on the absorbed dose and thermal stimulation in the crystal. ${ }^{41}$

The OSLD can be read by subsequent stimulation with light of a certain wavelength, $\lambda_{\text {stim }}$, which stimulates the electron into the conduction band where it is free to move through the crystal. If it reaches the trapped hole, the recombination process creates a defect in the excited state, which emits light of wavelength, $\lambda_{o s L}$, after relaxation to the ground state. Figure 2-1 outlines the basics of how an OSL material can be used as a dosimeter. 
(a) excitation

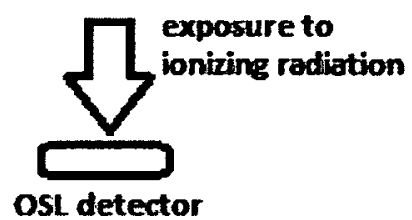

OSL detector

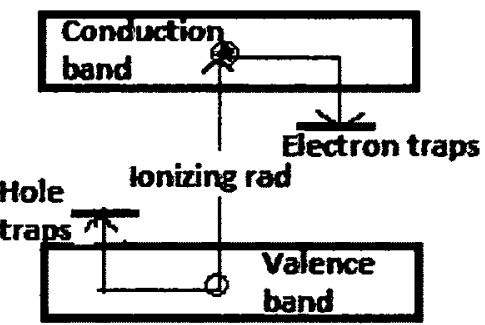

(b) latency

OSL detector
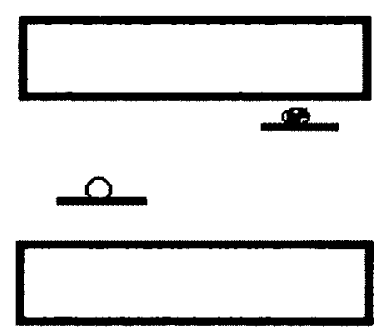

(c) stimulation

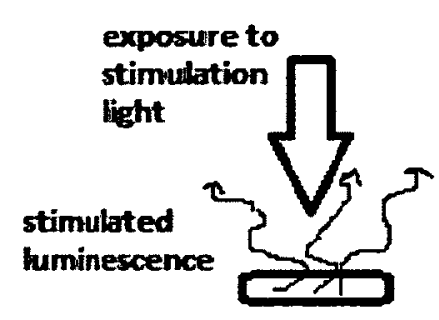

OSL detector

Figure 2-1: Representation of the OSL process. (a) excitation process describes period in which the dosimeter is worn by user, or exposed to an unknown radiation beam to be characterized; (b) latency process describes period in which the dosimeter is transported to laboratory and stored after exposure to radiation; (c) stimulation and readout process which gives information stored in detector for the radiation field.

\subsection{Rate equations for OSL process}

The transition of charges between energy levels during irradiation and the optical stimulation after illumination can all be described by a series of simultaneous non-linear, coupled rate equations. Several simplifying assumptions are introduced to arrive at analytical expressions to model and explain several aspects of OSL processes. A few of the assumptions made to set up and numerically solve these equations include:

- charge recombination occurs by charge transport via the delocalized energy bands; 
- the process is quasi-static, i.e., at any instant the system is in quasi-equilibrium (process is slow such that at any instant, concentrations of charges in the conduction band and valence band remain relatively small).

\subsubsection{One Trap-One Recombination center (OTOR) model}

A simplified TL/OSL model consists of the delocalized energy bands (valence and conduction band), and just two types of discrete defect levels: one is the electron trap acting as trapping center and the other, a hole trap acting as the recombination center. This model is usually referred to as the One Trap-One Recombination center (OTOR) model. ${ }^{41,63}$ Figure 2-2 is a schematic view of the OTOR model.

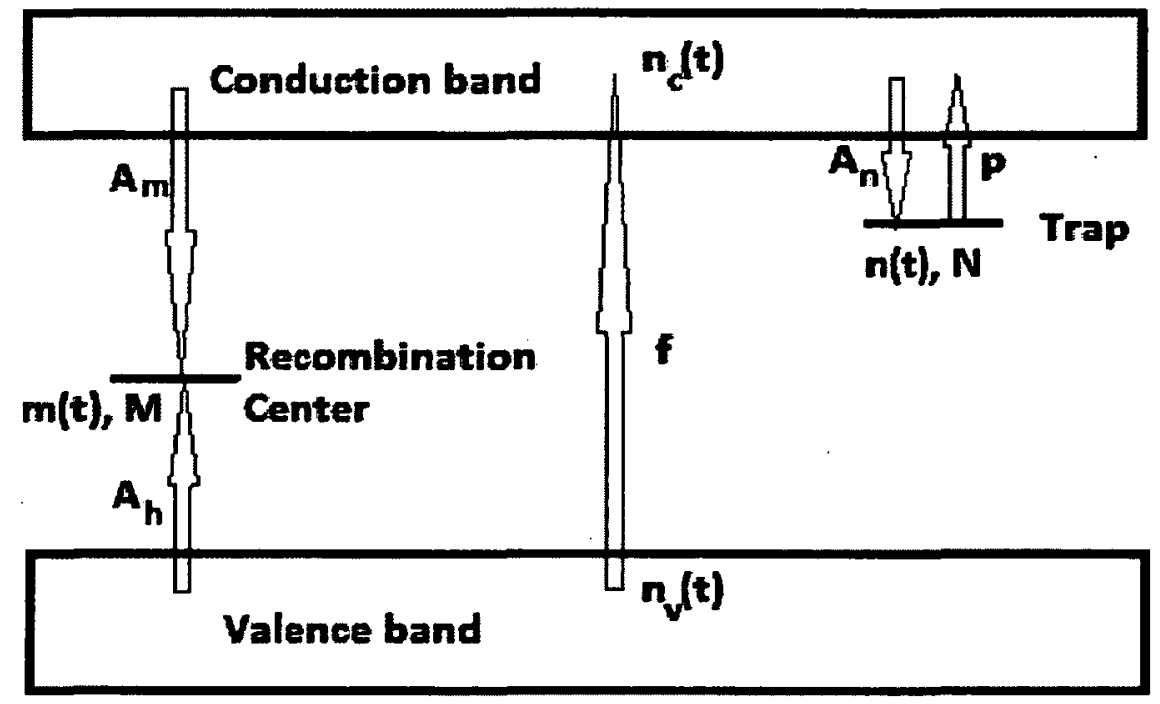

Figure 2-2: Schematic representation of the OTOR model. The parameters are explained in the text.

The parameters are represented as:

- $n(t)=$ concentration of trapped electrons $\left(\mathrm{m}^{-3}\right)$

- $m(t)=$ concentration of trapped holes $\left(\mathrm{m}^{-3}\right)$

- $n_{c}(t)=$ concentration of electrons in conduction band $\left(\mathrm{m}^{-3}\right)$ 
- $n_{v}(t)=$ concentration of holes in valence band $\left(\mathrm{m}^{-3}\right)$

- $p=$ probability rate of optical stimulation $\left(\mathrm{s}^{-1}\right)$

- $N=$ total concentration of trapping center $\left(\mathrm{m}^{-3}\right)$

- $M=$ total concentration of recombination center $\left(\mathrm{m}^{-3}\right)$

- $f=$ rate of electron-hole pairs created per unit volume $\left(\mathrm{m}^{-3} \mathrm{~s}^{-1}\right)$

- $A_{n}=$ probability rate of electron capture from conduction band $\left(\mathrm{m}^{3} \mathrm{~s}^{-1}\right)$

- $A_{m}=$ probability rate of electron recombination $\left(\mathrm{m}^{3} \mathrm{~s}^{-1}\right)$

- $A_{h}=$ probability rate of hole capture $\left(\mathrm{m}^{3} \mathrm{~s}^{-1}\right)$

Based on the model described above, we can write the rate equations. First, the rate of change in the concentration of trapped electrons, $d n / d t$ is equal to the rate of electrons being captured minus the rate of electrons escaping the trapping centers $n(t) p$, i.e.

$$
\frac{d n(t)}{d t}=[N-n(t)] A_{n} n_{c}(t)-n(t) p .
$$

The rate of variation in the trapped hole concentration, $d m / d t$, can similarly be written as

$$
\frac{d m(t)}{d t}=[M-m(t)] A_{h} n_{v}(t)-m(t) A_{m} n_{c}(t) .
$$

From charge conservation:

$$
n_{c}+n=n_{v}+m,
$$

while $d n_{d} / d t$ and $d n_{\sqrt{ }} / d t$ can be written as

$$
\begin{gathered}
\frac{d n_{c}(t)}{d t}=f-\frac{d n(t)}{d t}-m(t) A_{m} n_{c}(t), \\
\frac{d n_{v}(t)}{d t}=f-[M-m(t)] A_{h} n_{v}(t) .
\end{gathered}
$$

Invoking quasi equilibrium conditions, where $n_{c}<<n, m$; such that $d n_{d} d d \cong 0$ and $d n_{\sqrt{ }} / d t$ $\cong 0$. Under these assumptions, we arrive at:

$$
n_{c}(t)=\frac{f+n(t) p}{[N-n(t)] A_{n}+m(t) A_{m}}
$$


and

$$
\begin{gathered}
\frac{d n(t)}{d t}=[N-n(t)] A_{n} n_{c}(t)-n(t) p \\
\frac{d m(t)}{d t}=f-m(t) A_{m} n_{c}(t)
\end{gathered}
$$

These equations can be used to represent processes taking place in the crystal during irradiation and optical stimulation. During irradiation, $f \neq 0$, whereas when there is no radiation, $f=0$. The set of differential equations can be solved numerically using mathematical software packages or user-specific codes. ${ }^{39,64}$

Now, assume optical stimulation of traps with no radiation (i.e. $f=0$ ), and that transitions to the valence band do not occur any time. We have $n_{v}=0$, and from Eq. (2.8):

$$
\frac{d m(t)}{d t}=-m(t) A_{m} n_{c}(t)
$$

while recalling Eq. (2.3) and Eq. (2.4), we arrive at:

$$
\frac{d n_{c}}{d t}=-\frac{d n}{d t}+\frac{d m}{d t}
$$

From Eq. (2.1), $p$ is the rate of stimulation (units of $\mathrm{s}^{-1}$ ) of electrons from electron trap, which is related to the incident photon intensity, $\Phi$, and the photo-ionization crosssection, $\sigma$, by the relationship:

$$
p=\Phi \sigma
$$

Considering quasi-static population of the free electrons in the conduction band $\left(d n_{d} d d t\right.$ $<<d n / d t ; d m / d t$ ), we arrive at:

$$
\frac{d m}{d t}=\frac{d n}{d t}
$$

Another major assumption that can be made is slow retrapping of electrons and holes [i.e. $\left.n_{c} A(N-n)<<n p ; n_{c} A m(t)\right]$. This leads to: 


$$
\frac{d n}{d t}=-n p=\frac{d m}{d t}
$$

The solution of Eq. (2.13) is thus:

$$
n=n_{o} e^{-t p}
$$

such that:

$$
I_{O S L}=n p=n_{o} p e^{-t p}=I_{o} e^{-t / \tau_{d}}
$$

where $I_{o}$ is the initial OSL intensity at $t=0$ and $\tau_{d}$ is the OSL decay constant. Hence, this first-order model leads to an exponentially decaying OSL intensity as the constant stimulation light intensity is applied. ${ }^{65}$

Note that it was assumed that the optical stimulation intensity and wavelength are fixed. In this case, the recorded luminescence is known as continuous wave OSL or CWOSL. However, for a linear increase in optical stimulation intensity, the luminescence recorded is known as linear modulation OSL or LM-OSL. Other stimulation schemes employed are: pulsed OSL or POSL and exponential modulation. In this work we are concerned with CW-OSL only. Yukihara and McKeever (2011) ${ }^{39}$ present a detailed description of the other stimulation modes.

\subsubsection{Multiple traps and multiple center defect model}

In practice, experimental CW-OSL decay curves show wide varieties of curve shapes, which do not conform to the simple exponential decay description. A reason for that is the presence of other active traps and recombination centers leading to two or more electron traps releasing trapped charges, each at its own rates and photo-ionization cross-

sections. ${ }^{41}$ As a result, such model needs to be dealt with separately. 


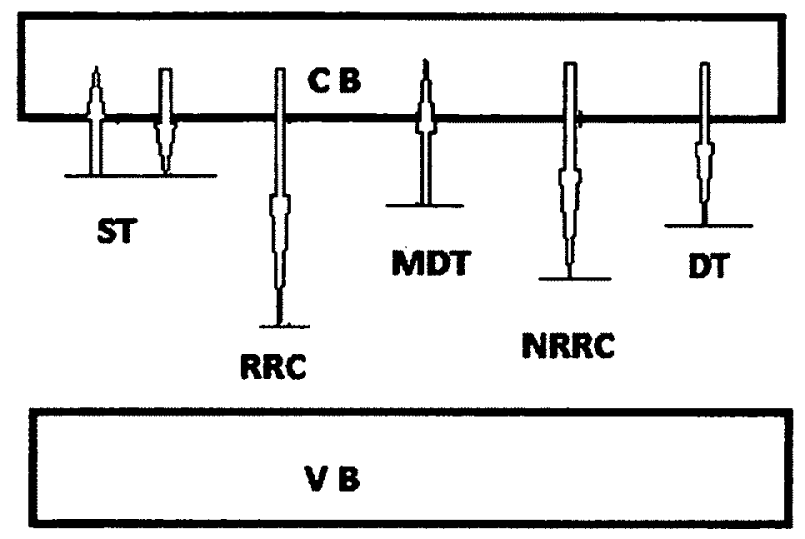

Figure 2-3: A model showing the three-trap, two-center model of the $\mathrm{Al}_{2} \mathrm{O}_{3}$ : C crystal (following McKeever et al. $1997^{64}$ and Botter-Jensen et al. 2003 ${ }^{41}$ ). CB-conduction band; VB- valence band; STshallow trap; MDT-main dosimetry trap; DT-deep trap; RRC-radiative recombination center; and NRRC- non-radiative recombination center.

A model introduced by McKeever et $a l .{ }^{64,65}$ (see Figure 2-3), shows a generic OSL material consisting of a shallow trap (ST) into which electrons may be trapped during irradiation and optical stimulation and from which electrons may be thermally or optically released; a main dosimetry trap (MDT) from which electrons are optically stimulated; a deep trap (DT) into which electrons may be trapped and remain localized; a radiative recombination center $(\mathrm{RRC})$ into which electrons may recombine with trapped holes to produce OSL; and a non-radiative recombination center (NNRC) at which recombination can also occur without producing luminescence. The MDT is thermally stable at room temperature and charges only escape by optical stimulation. The DT only competes for charges released to the conduction band. As a result, DT can cause an overall reduction in OSL intensity by capturing charges that would have recombined producing OSL.

This simplistic model encompasses many of the features that are found in an OSL material. It should be noted that these transitions are those that occur during the optical 
stimulation process only. The rate equations describing the flow of charges into and out of the various traps and recombination centers form a set of six, coupled non-linear equations. The behavior of different materials can be explained solving these rate equations numerically. Examples of such procedure were conducted by McKeever et al. $1997^{65}$ and Bøtter-Jensen et al. $2003^{66}$ and the trends can be compared with experimental observations.

At low temperatures, a reduced OSL signal was obtained while at higher temperatures the OSL intensity was much higher. It was observed that the OSL decay after the initial increase appears approximately exponential at shorter times but departs from an exponential decay at longer times. ${ }^{39,65}$ It was suggested that the reason for the non-exponential decay may be the competition between the three traps (ST, MDT and DT) and recombination centers (RRC, NRRC) during stimulation.

Different factors influence the temperature dependence of the OSL signal. The OSL may be influenced due to the thermal quenching of the luminescence; competition by shallow traps (ST) and thermally assisted optical stimulation. Thermal quenching is the loss of luminescence intensity due to increasing temperatures. It has been explained to be due to two main factors: the decrease in the luminescence quantum efficiency (see Section 2) with increasing temperature (Mott-Seitz model), and a reduction in the concentration of recombination centers during heating (Schön-Klasens model). ${ }^{39,41}$ However for OSL measurements carried out in a temperature range with luminescence efficiency $\sim 1$, thermal quenching is negligible. ${ }^{39}$

Competition for charges by ST at low temperatures leads to capture of electrons at the ST instead of recombining at the centers, hence leading to lower OSL intensity than 
when the ST was full or absent. ${ }^{39}$ However at high temperatures, the electrons captured by ST are thermally stimulated due to heat and are quickly released by the ST to the conduction band, leading to recombination with trapped holes and hence an increase in the OSL intensity.

\subsection{OSL readout process}

CW-OSL is the simplest and most widely used approach of OSL readout. It uses light of constant intensity to stimulate the OSLD. Since the OSL light has to be discriminated against the stimulation signal, this modality is based on the separation of the wavelengths using optical filters. Hence, the transmission band of the optical filters must not overlap with the spectrum of the stimulation light.

A simple description of the CW-OSL curve has been discussed in Section 2.2 for a first-order recombination process, represented by Eq. (2.15). In this simplistic model, the rate of decay of the OSL curve depends only on $p$ and not on $n_{o}$. Hence, the shape of the OSL decay curve is independent of dose. In contrast, the total area under the OSL curve depends only on $n_{o}$ and not on $p$ evidently from the relationship

$$
\int_{0}^{\infty} I_{O S L}(t) d t \propto \int_{0}^{\infty} n_{o} p e^{-p t} d t=n_{o} .
$$


The relationship above indicates that the total area under a CW-OSL curve following a first-order kinetics model, is independent of the OSL stimulation intensity $p .^{39,41}$ Thus, it can be inferred that for an OSLD stimulated with constant intensity, the absorbed dose can be related to the OSL curve integrated for any given fixed duration. An important practical implication is that provided the stimulation duration produces sufficient sensitivity and precision, the stimulation can be interrupted; leaving the remaining trapped charges for future stimulation. Hence, the absorbed dose by the OSLDs can be preserved and re-evaluated making the OSLDs re-readable. ${ }^{39}$

\subsection{Dose measurement formalism for clinical dosimetry}

The formalism presented in this section follows a proposed formalism by Yukihara and McKeever $2011,{ }^{39}$ based on terminologies used to describe the characteristics of radiation dosimeters in clinical dosimetry for radiation therapy. ${ }^{18,35,67}$

\subsubsection{The OSL signal, $S$}

The dose information stored in an OSLD can be read using a PMT operating in photon counting mode. Hence, the OSL signal $S$ is the number of pulses (counts) over a certain stimulation interval, with the correction for the background signal due to the instrument, such that;

$$
S=S_{\text {raw }}-S_{b g}
$$

where $S_{\text {raw }}$ is the uncorrected raw OSL signal and $S_{b g}$ is the estimated instrumental background. The instrumental background can be estimated using un-irradiated control OSLDs. 


\subsubsection{The OSL reading, $M_{\text {raw }}$ OSL}

$M_{\text {raw }}{ }^{\text {OSL }}$ is the user-defined reading of the OSLD which can be either the OSL signal $S$ or the reading after correction by the detector sensitivity factors, calibration factors or coefficients. $M_{\text {raw }}{ }^{\text {OSL }}$ can be measured as the absorbed-dose, depending on the calibration of a set of detectors exposed to the same conditions.

\subsubsection{The corrected $O S L$ reading, $M^{\text {OSL }}$}

$M^{\mathrm{OSL}}$ is the measured result after the correction of the OSLD reading for the intrinsic influence quantities of the dosimeter. These intrinsic influence quantities are characteristic of the material used for the OSLD. The influence quantities may arise from environmental factors like temperature during irradiation or readout; from the detector itself such as fading of OSL signals and linearity of dose response; or from the radiation field such as beam quality, field sizes, dose rate, and depth in phantom.

$M^{\mathrm{OSL}}$ can be calculated using a model function for the measurement as

$$
M^{\mathrm{OSL}}=\left(M_{\mathrm{raw}}^{\mathrm{OSL}}-M_{\mathrm{raw}, 0}^{\mathrm{OSL}}\right) \prod_{i} k_{i},
$$

where $k_{i}$ is the correction factor for the $i$ th influence quantity, $M_{\mathrm{raw}}^{\mathrm{OSL}}$ is the user defined OSL reading and $M_{\mathrm{raw}, 0}^{\mathrm{OSL}}$ is the background dose which is obtained with the OSL reading of control dosimeters. A few of the correction factors related to the dosimeters and environmental factors include:

i. Correction factor for fading $k_{f}$ : This can be obtained by comparing the OSL response of a dosimeter at a particular time $t$, to the response at a reference time $t_{o}$, after exposure of the same dosimeter to an identical or similar dose $D$ assuming other correction factors are unity. Hence, 


$$
k_{f}(t)=\frac{\left(M^{O S L} / D\right)_{t_{0}}}{\left(M^{O S L} / D\right)_{t}} .
$$

ii. Correction factor for non-linearity $k_{D}$ : If the dose response of a dosimeter is nonlinear, the non-linearity factor can be determined by a factor $k_{D}=k(\mathrm{D})$. The factor is determined as the ratio of the corrected OSL reading at a reference dose range where the dosimeter response is linear, divided by the OSL reading at an arbitrary dose. This can be represented by

$$
k_{D}(D)=\frac{\left(M^{O S L} / D\right)_{D=D_{O}}}{\left(M^{O S L} / D\right)_{D}} .
$$

Other correction factors include correction factors for the loss of the OSL signal after successive readouts of the same dosimeter and correction factors dependent on the characteristics of the irradiation beam quality.

\subsubsection{The calibration coefficient $N_{D, w, Q \text { ref }}$}

To determine the absorbed-dose delivered by a dosimeter from a particular beam quality $Q$, the dosimeter has to be calibrated in reference to the absorbed-dose-to-water for a reference beam quality $Q_{r e f}$ in a standards dosimetry laboratory at reference conditions as defined by the standards laboratory. The calibration coefficient $N_{D, w, Q r e f}$ that relates the absorbed-dose by the dosimeter $D_{w, Q r e f}$ to this standard reference condition is represented as

$$
N_{D, w, Q_{r e f}}^{O S L}=\frac{D_{w, Q_{r e f}}}{M_{Q_{r e f}}^{O L L}}
$$

where $M_{Q_{\text {ref }}}^{O S L}$ is the OSL reading after correction for all influence quantities. The calibration coefficient is only dimensionless if $M_{Q_{r e f}}^{O S L}$ has same units as $D_{w . Q r e f}$ If $M_{Q_{r e f}}^{O S L}$ 
has arbitrary units ( $\mathrm{rdg}$ ), then $N_{D, w, Q_{r} f}^{O S L}$ is in fact a calibration coefficient with units Gy/rdg.

\subsubsection{Beam Quality correction factor $k_{Q Q}$}

The absorbed-dose-to-water at conditions different from reference conditions, established by the standards laboratory is given by the relationship:

$$
D_{w, Q}=M_{Q}^{O S L} N_{D, w, Q \text { ref }}^{O S L} k_{Q, Q_{\text {ref }}}
$$

where $D_{w, Q}$ is the absorbed-dose-to-water for a beam quality $Q$ different from the reference beam quality $Q_{r e f}, M_{Q}^{O S L}$ is the fully corrected OSL reading for the dosimeter at the beam quality $Q$, and $k_{Q, Q r e f}$ is the beam quality correction or conversion factor ${ }^{2,39}$ defined as

$$
k_{Q, Q_{\text {ref }}}=\frac{N_{D, w, Q}^{O S L}}{N_{D, w, Q_{\text {ref }}}^{O S L}}=\frac{\left(M_{Q r e f}^{O S L} / D_{w, Q_{\text {ref }}}\right)}{\left(M_{Q}^{O S L} / D_{w, Q}\right)}
$$




\section{Materials and Methods}

\subsection{Introduction}

We characterized the OSLDs for: dose response, signal loss after sequential readouts, optical bleaching techniques, system reproducibility, fading of OSL signal postirradiation, regeneration of OSL signals in dark and the sensitivity of the OSLDs to accumulated dose and bleaching light wavelengths.

\subsection{Dosimeters and dosimeter preparation}

OSLDs of the type nanoDot, manufactured by Landauer Inc. (Glenwood, IL, USA), were used throughout this study. The nanoDot OSLD's sensitive volume is $\mathrm{Al}_{2} \mathrm{O}_{3}: \mathrm{C}$ with dimensions of $5 \mathrm{~mm}$ diameter, and $0.3 \mathrm{~mm}$ thick. The sensitive volume is enclosed in a light-tight plastic carrier with unique serial numbers and bar codes, which can be used to specify the sensitivity for each nanoDot OSLD. The barcode can also be used to track and identify every nanoDot. 'The plastic light-tight carrier is of $10 \mathrm{~mm}$ width, $10 \mathrm{~mm}$ height, and $2 \mathrm{~mm}$ thick with an opening to expose the enclosed $\mathrm{Al}_{2} \mathrm{O}_{3}: \mathrm{C}$ sensitive volume when required as seen in Figure 3-1.

Screened nanoDot OSLDs acquired from Landauer Inc., which were pre-selected by the manufacturer to have batch reproducibility within $\pm 3 \%$, were used throughout this study. We did not perform any pre-treatment of the OSLDs before their first use. 


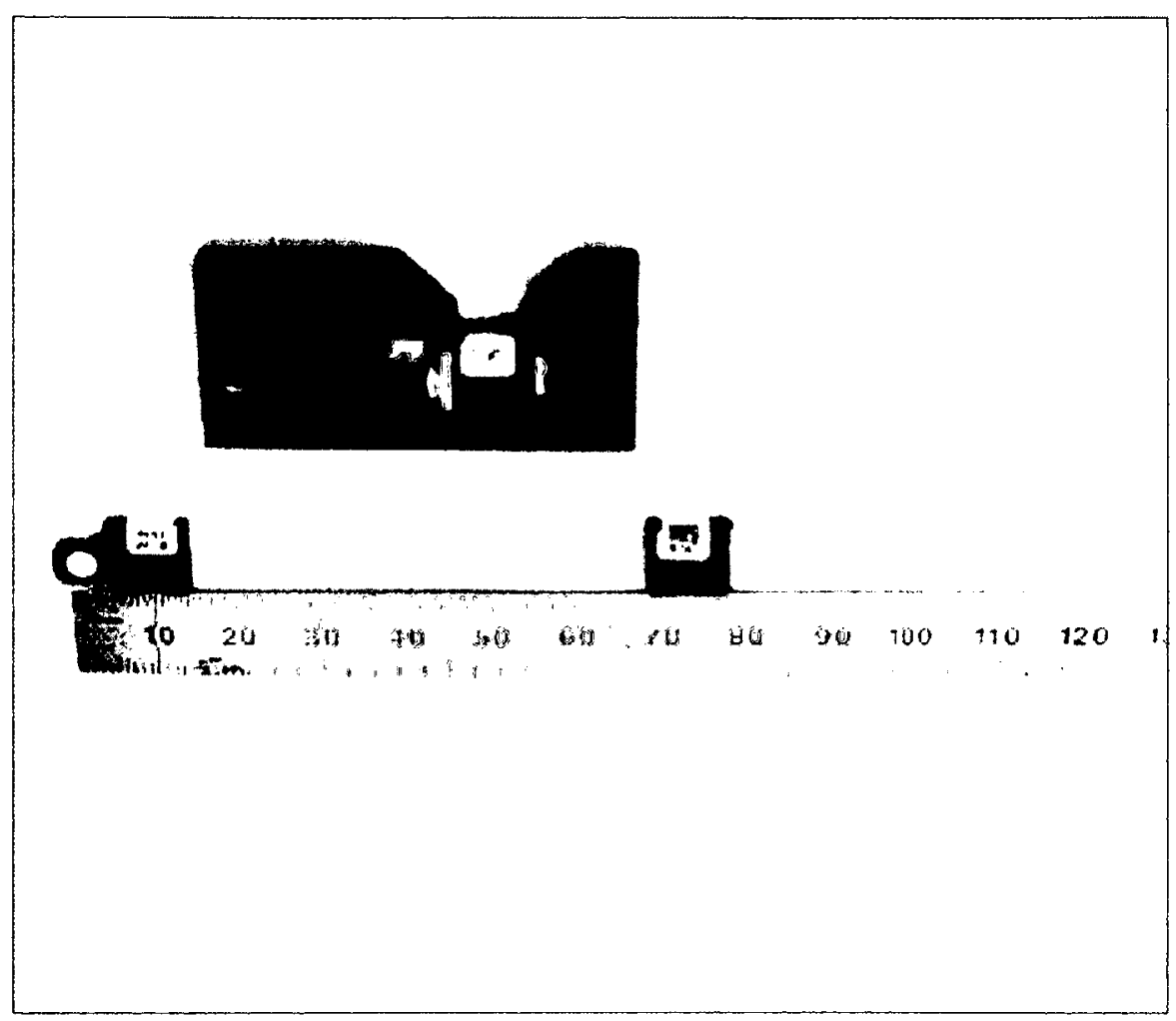

Figure 3-1: Landauer nanoDot OSLDs and adapter (top) used to hold the OSLDs for readout in the MicroStar reader. The plastic casing has dimensions of $10 \mathrm{~mm} \times 10 \mathrm{~mm} \times 2 \mathrm{~mm}$, while the sensitive volume of the dosimeter is $5 \mathrm{~mm}$ in diameter and $0.3 \mathrm{~mm}$ thick. The dosimeter on the left shows the exposed sensitive volume from the light-tight casing.

\subsection{Irradiations}

The irradiations were carried out at The Ottawa Hospital Cancer Centre (TOHCC, Ottawa, Canada) in a 6 MV x-ray beam (Primus Siemens AG, Munich, Germany). For the experiments, sets of 9 nanoDot OSLDs were exposed each to doses in the range from 0.1 to $10 \mathrm{~Gy}$. To minimize air gaps, we used a $6 \mathrm{~cm}$ plastic slab (Standard Grade Solid Water, Gammex, Middleton, WI, USA) with custom-made grooves for loading the nanoDot OSLDs as shown in Figure 3-2. 


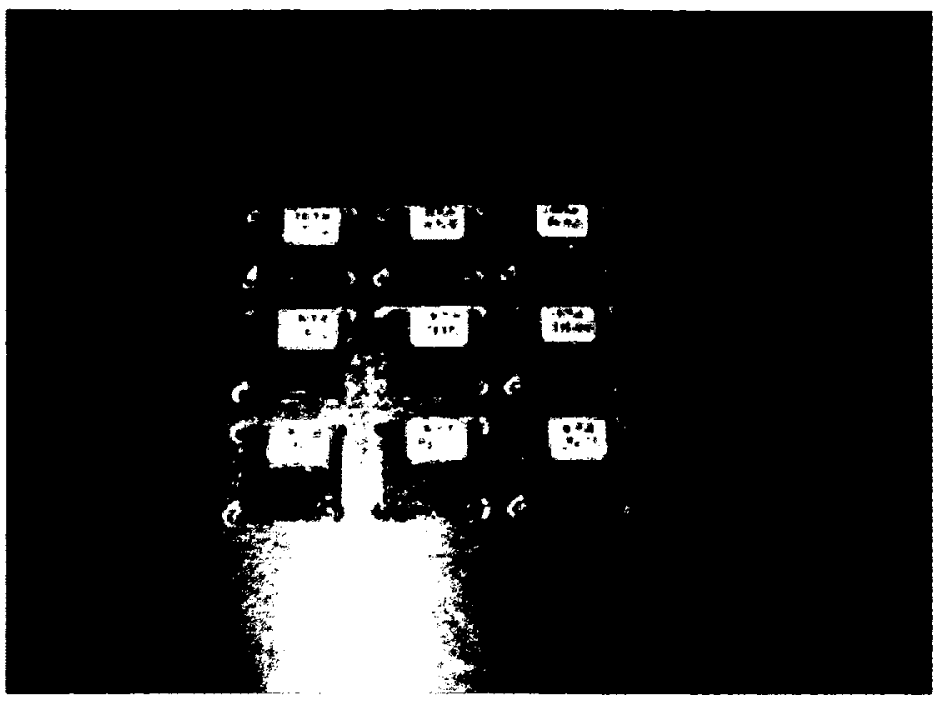

Figure 3-2: Grooved solid water phantom used for loading nanoDot OSLDs for irradiations.

The nanoDot OSLDs were exposed (barcode number face-up) in a solid water phantom at $5 \mathrm{~cm}$ water equivalent depth. Figure 3-3 illustrates the irradiation setup. The flatness and symmetry of the beam in which the dosimeters were exposed were $0.9 \%$ and $0.3 \%$, respectively. The non-uniformity of the beam at the OSLDs' position was not accounted for in our analyses. ${ }^{1}$

Beam calibration setup

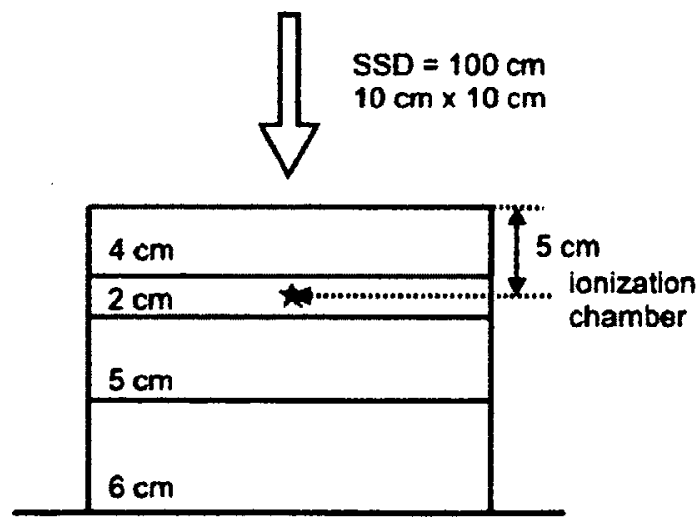

Irradiation setup
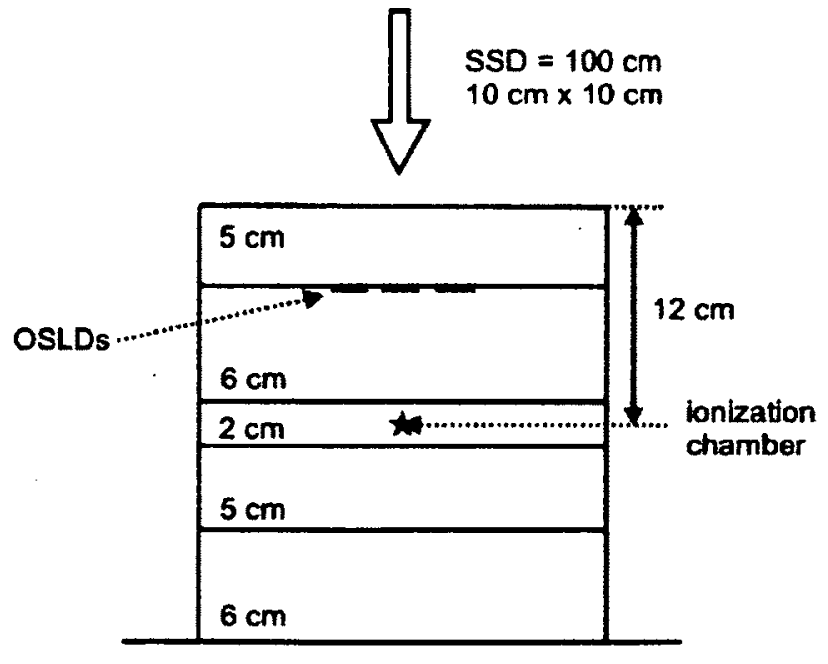

Figure 3-3: Calibration and irradiation setup. Irradiations were carried out in a $6 \mathrm{MV}$ photon beam at source to surface distance (SSD) of $100 \mathrm{~cm}$ and field size $10 \mathrm{~cm} \times 10 \mathrm{~cm}$. 
We used a cylindrical ionization chamber (NE2571, S/N 2025, Nuclear Enterprises, UK) calibrated in terms of absorbed-dose-to-water in a cobalt- 60 beam by the National Research Council of Canada (NRC, Ottawa, Canada) to determine the absorbed-dose-to-water, $D_{w}$ per monitor unit (MU) at the position in which the nanoDot OSLDs were exposed. The ionization chamber was used with an electrometer (NE2570, S/N 783, Nuclear Enterprises, UK), which was also calibrated by NRC, to obtain the raw charge readings. The dose was calculated based on the TG-51 reference dosimetry protocol, ${ }^{18}$

$$
D_{w}=M_{\text {raw }} N_{D, w} k_{Q} P_{T, P} P_{i o n} P_{p o l}
$$

where $M_{\text {raw }}$ is the uncorrected raw electrometer reading, $N_{D, w}$ is the absorbed-dose-towater coefficient; and $P_{T, P}, P_{i o n}, P_{p o l}$ are the temperature-pressure, ionization recombination and the polarity correction factors for the ionization chamber, respectively. For our purposes, OSLDs were calibrated against an ionization chamber as the reference instrument, which had been previously calibrated at NRC.

\subsection{OSL readout process}

The nanoDot OSLDs were read in a MicroStar OSLD reader (S/N 1340400, Figure 3-4), commercially manufactured by Landauer Inc. The reader operates in CW-OSL and has two readout modes: 'strong-stimulation' and 'weak-stimulation'. The strong-stimulation readout mode uses high stimulation intensity with 38 green light emitting diodes (LEDs), for readout of OSLDs exposed to doses smaller than approximately $0.1 \mathrm{~Gy}$; while the weak-stimulation mode uses low stimulation intensity with 6 LEDs for readout of detectors exposed to doses greater than approximately $0.1 \mathrm{~Gy}$. The reader was calibrated 
for both modes, using the standard (linear) calibration algorithm available with the software that is distributed with the MicroStar reader. It should be noted that the reader is programmed to automatically select the readout mode based on the PMT test counts during the readouts. Thus, the dose at which the reader changes from the strongstimulation to weak-stimulation mode depends on the sensitivity of the dosimeter.

The optical stimulation was produced by a combination of LEDs and a coloured glass band-pass filter (Schott OG-515, Schott AG, Mainz, Germany) producing a peak emission at $525 \mathrm{~nm} .{ }^{68}$ The OSL signal was measured using a PMT and band-pass filters (B370, Hoya Corporation, Tokyo, Japan and Schott BG-12) in front of the PMT. ${ }^{49,68}$ The stimulation time was $1 \mathrm{~s}$. This readout protocol assures the OSL signal is mainly due to the OSL emission at $420 \mathrm{~nm}$. Prior to each readout session, we verified the stability of the MicroStar reader using the position dials DRK, CAL and LED to monitor daily variations of the PMT counts in dark, PMT output and LED intensity, respectively. Throughout the course of our investigations, we did not observe significant variation in the stability of the reader.

For all measurements, OSLDs were read three times, with the average of the three readings taken as the OSLD signal. Figure 3-4 shows the MicroStar reader, with the reader slide open with the adapter for loading the nanoDot OSLDs for readout. 


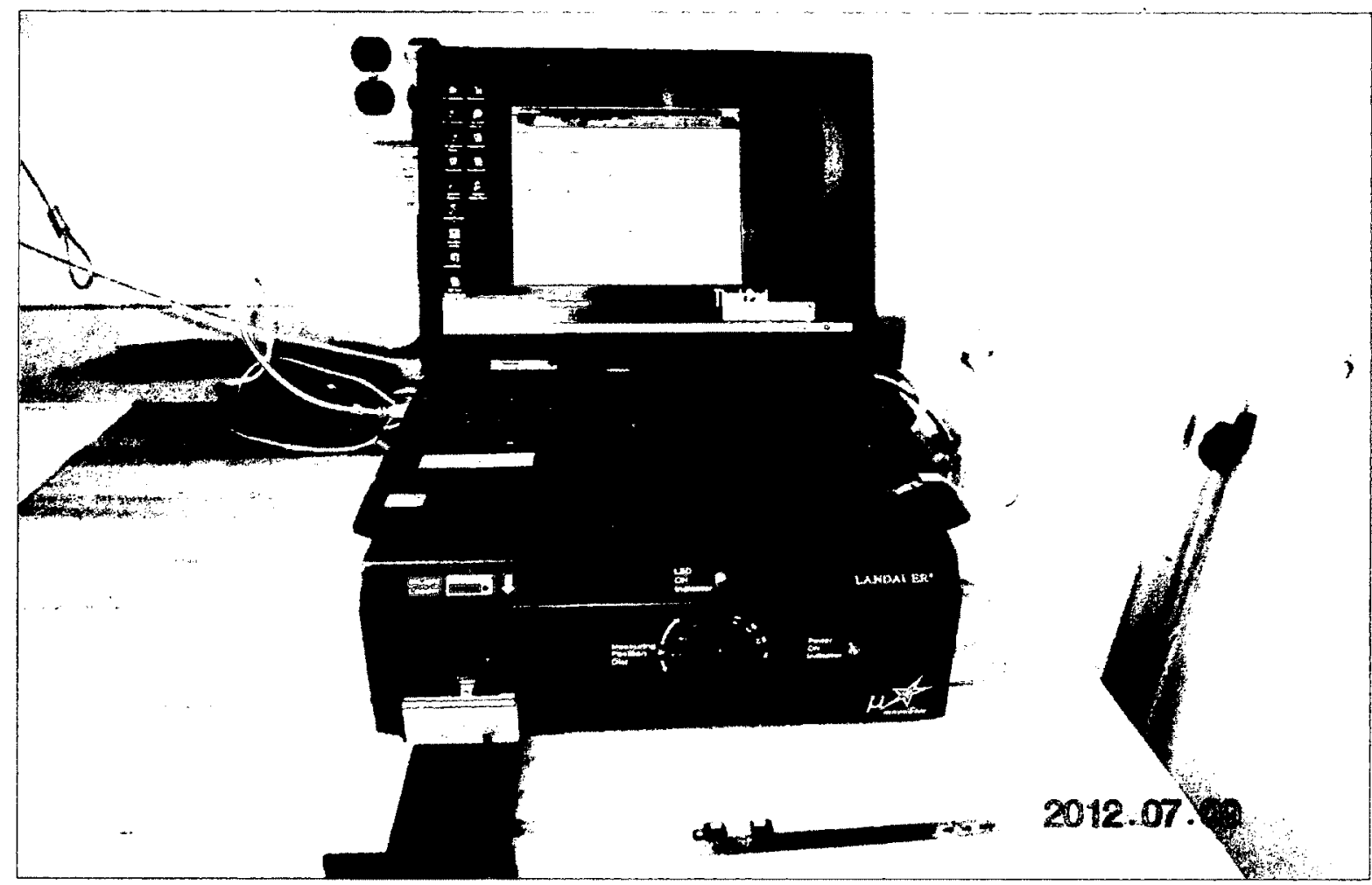

Figure 3-4: The InLight MicroStar reader with the open cassette for loading the nanoDot OSLDs. The reader comes with programmed software on the computer, for reading out the OSLDs.

\subsection{Basic Characterization of the OSL system}

\subsubsection{Dose response}

We exposed the nanoDot OSLDs to a dose range from 0.5 to $10 \mathrm{~Gy}$ to investigate the linearity of the signal as a function of dose. Recalling from Section 1.7, non-linear responses have been reported at different doses. $24,33,55,69,70$

We assumed that the nanoDot OSLD's signal $S$ is linear up to a dose of $1 \mathrm{~Gy}$. Then, we fit the data in the range of doses up to $1 \mathrm{~Gy}$, to a linear function passing through the origin ( $S_{\text {linear }}=A D$, where $A$ is the slope and $D$ is the dose). To quantify the amount of deviation from the linear behavior, we analyzed the dose response in terms of the supralinearity index, defined as the signal per dose $(S / D)$ at any dose $D$, divided by the signal per dose if the OSLDs were linear $\left(S_{\text {linear }} / D\right)$. Thus, the supralinearity index is 
given by:

$$
\frac{(S / D)}{\left(S_{\text {linear }} / D\right)}=\frac{(S / D)}{A}
$$

For the dose response experiments, two nanoDot OSLDs were exposed to each dose. Each data point thus represents the average of two dosimeters, in which each OSLD was read three times.

\subsubsection{Loss of OSL signal as a function of sequential readouts}

Each reading of the OSLD partially reduces the trapped charges in the crystal, leading to a decay and reduction of the OSL signal. However, since OSLDs can be read multiple times following irradiation; we studied the effect of successive readouts on the OSL signal. To determine the dependence of the signal loss on absorbed dose, different nanoDot OSLDs were exposed to doses of $0.1,0.2,0.5,1$ and $10 \mathrm{~Gy}$. A few hours after irradiation, each OSLD was then read 50 or 100 times successively in the MicroStar reader to determine the rates of loss of the OSL signal for the different doses and individual detectors. Two detectors were evaluated for doses of $0.1,1$ and $10 \mathrm{~Gy}$ and one detector was evaluated for the other doses.

Linear and exponential models were used to determine the rate of loss of the OSL signals as functions of sequential readouts for both readout modes: weak- and strongstimulation modes, respectively. The uncertainty in the rate of loss values was determined using standard procedures of the regression theory. When two detectors were evaluated for the same dose, we reported the average of the two rates of loss values and the uncertainty as the standard deviation of the two rates of loss values, combined with the errors determined using the regression theory. 
The loss of OSL signal as a function of sequential readouts could depend on the MicroStar reader unit, dose and individual detectors. To verify that the loss of OSL signal as a function of sequential readouts does not introduce additional uncertainties in our data, it is also important to characterize it. ${ }^{1}$

\subsubsection{Fading of the OSL signal}

The fading effect is the decay of the OSL signal in the dark at room temperature. This could lead to transient signals in OSLDs; hence, four nanoDot OSLDs were studied by irradiating them with doses of 1 and $10 \mathrm{~Gy}$, and read out as soon as $60 \mathrm{~s}$ immediately post-irradiation in the MicroStar reader at room temperature. The OSL signals were normalized to the last readout to monitor any dependence of the fading effect as a function of dose. ${ }^{1}$

The study was carried out using two different nanoDot OSLDs for each dose. The nanoDot OSLDs were readout as a function of post-irradiation time. To obtain a data point for the fading curve we read each nanoDot only once with error bars representing the statistical fluctuations in the PMT counts. In the fading experiments, the loss of the OSL signal after successive readouts was not accounted for because the correction was negligible for the investigated doses.

A linear-exponential model was fit to the fading data for the doses, using a nonlinear regression represented as ${ }^{1}$

$$
y(t)=A+B t+C \exp \left(-\ln 2 \cdot t / T_{1 / 2}\right),
$$

where $y(t)$ is the OSLD signal at time $t$ post-irradiation, after normalizing to the last readout; while $A, B$ and $C$ are fitting parameters and $T_{1 / 2}$ is the half-life of the fading. 
Fitting was performed using a non-linear least square fitter that is based on the Levenberg-Marquardt algorithm. The uncertainties (errors) in the fitting parameters were determined as the square root of the respective diagonal element of the variancecovariance matrix.

The fading of the OSL signal could depend on dose. To verify that the fading of the OSL signal does not introduce additional uncertainties in our data, it is also important to characterize it.'

\subsubsection{Sensitivity changes}

$\mathrm{Al}_{2} \mathrm{O}_{3}: \mathrm{C}$ OSLDs can be effectively reset by annealing the dosimeter to $900^{\circ} \mathrm{C}$ for $15 \mathrm{~min}$. This thermal treatment is sufficient to effectively empty all the electron traps (ST, MDT and DT) and re-establish the initial un-irradiated conditions of the material. ${ }^{1,64,71}$ However, nanoDot OSLDs are made of $\mathrm{Al}_{2} \mathrm{O}_{3}: \mathrm{C}$ powder fixed on a transparent plastic slide. Because of the presence of the plastic, it is not possible to apply a thermal treatment to the OSLDs. Thus, the alternative is to use light to bleach the OSLDs.

Illumination is effective in emptying the ST and MDT but not the DT. The inability to completely empty the DT causes changes in the sensitivity of the OSLD as a function of accumulated dose. Thus, if one wants to re-use the dosimeters in a multipleuse protocol to minimize costs, it would be important to determine ways to minimize and account for the changes in sensitivity of the dosimeters in the calibration protocol. We

present an experimental investigation of the changes in sensitivity of $\mathrm{Al}_{2} \mathrm{O}_{3}: \mathrm{C}$ nanoDot OSLDs exposed to accumulated doses of a $6 \mathrm{MV}$ clinical linac photon beam and to different bleaching light sources. 
Bleaching was performed in a custom-built unit (Figure 3-5), ${ }^{1}$ which contained four $26 \mathrm{~W}$ fluorescent light bulbs (General Electric, Fairfield, CT). Bleaching inside the unit was performed in two modes: (i) directly under the lamps, and (ii) with the aid of a long-pass optical filter (GG-495, Schott AG, Mainz, Germany). The long-pass filter was placed over the nanoDot OSLDs inside the bleaching unit, completely blocking wavelengths below $495 \mathrm{~nm}$. The experimental conditions using mode (ii) were very similar to the "yellow" bleaching condition used by Umisedo et al. $2010 .^{72}$

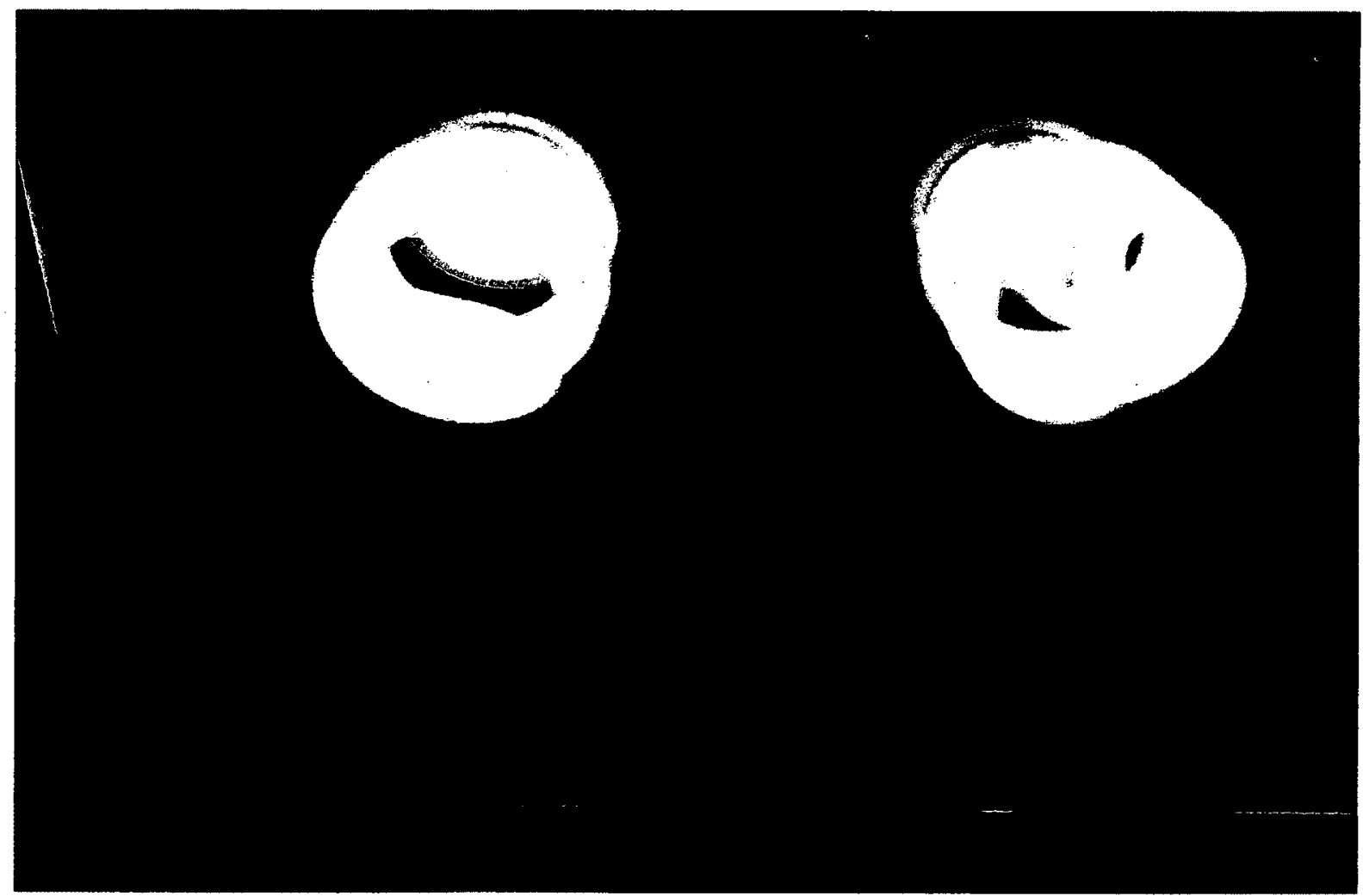

Figure 3-5: Custom-built bleaching unit containing four $26 \mathrm{~W}$ fluorescent bulbs to bleach the nanoDot OSLDs. In this picture, a long-pass filter (Schott GG-495) was placed over the nanoDot OSLDs to block wavelengths below $495 \mathrm{~nm}$.

The spectra and power of the bleaching light sources, i.e., without and with the filter, were measured using a portable spectrometer (HR4C2745, Ocean Optics, Dunedin, FL) and power meter (2832-C, Newport, Irvine, CA), respectively. To measure the power, we placed the power meter detector at different positions under the lamps and 
determined average powers at $542 \mathrm{~nm}$ wavelength of $(5.62 \pm 0.17) \mu \mathrm{W} / \mathrm{cm}^{2}$ and $(3.29 \pm$ $0.09) \mu \mathrm{W} / \mathrm{cm}^{2}$ for bleaching modes (i) and (ii), respectively. Figure 3-6 shows a plot of the spectra with and without the long-pass filter. ${ }^{1}$

It is also important to report the bleaching temperature because it might affect the efficiency of emptying the charge traps, i.e., the bleaching time required to attain a desired level of residual OSL signal. Other temperature effects such as changes in sensitivity of the detectors may be negligible for the range of bleaching temperatures (up to about $80^{\circ} \mathrm{C}$ ) that can be used without damaging the plastic carrier of the nanoDot OSLDs. During bleaching, the maximum temperatures at the OSLD position were measured as $(60 \pm 1)^{\circ} \mathrm{C}$ and $(40 \pm 1)^{\circ} \mathrm{C}$, for bleaching modes (i) and (ii), respectively. ${ }^{1}$

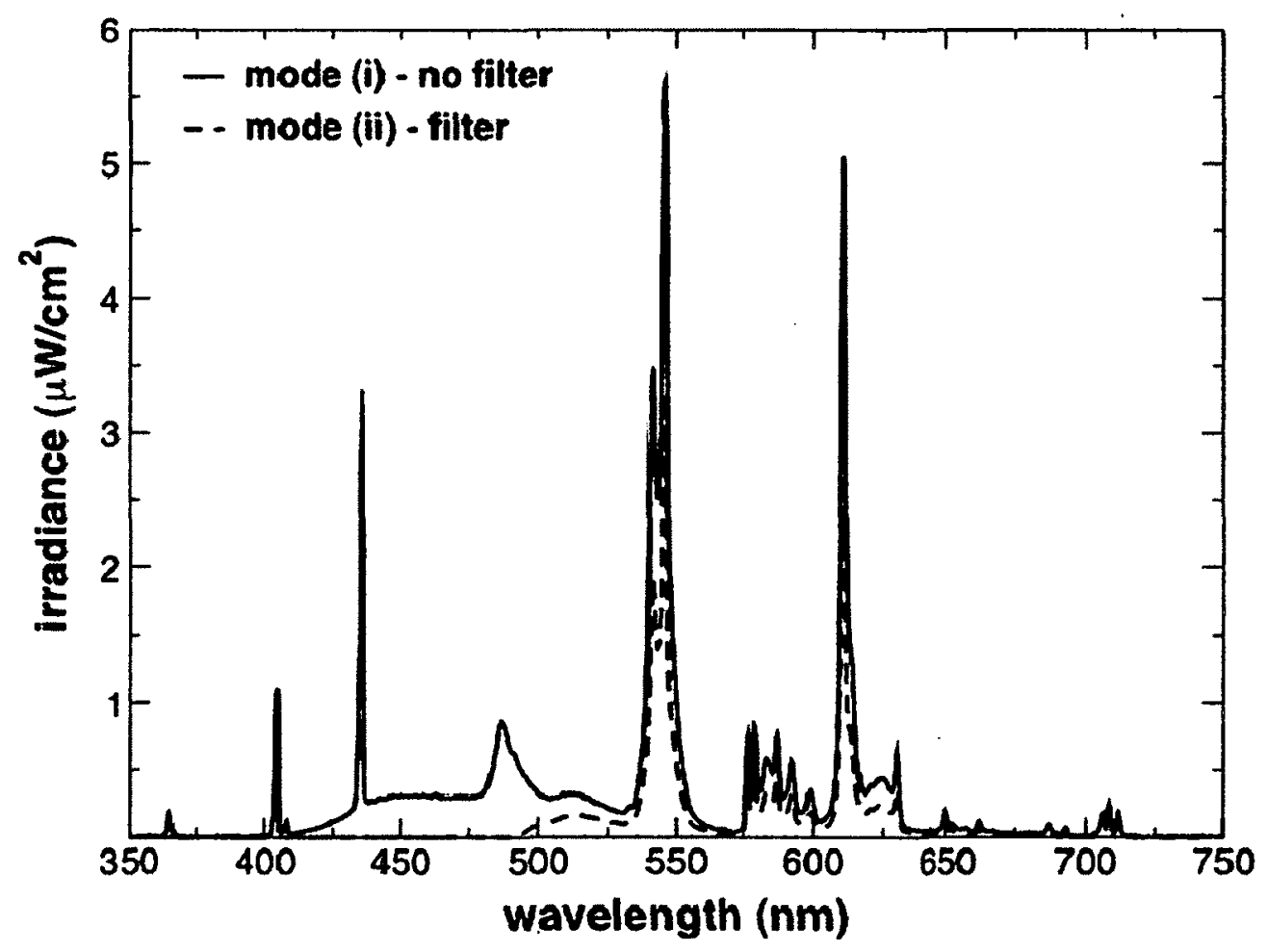

Figure 3-6: Emission spectra of the custom-built bleaching unit. The solid line is the spectrum of direct illumination (bleaching mode i) and dashed line is the spectrum using a long-pass filter (Schott GG-495) between the light source and light spectrometer (bleaching mode ii). 
Twenty-four nanoDot OSLDs were used to study their response to accumulated doses. The OSLDs were subjected to cycles of irradiation-readout-bleaching-readout, shown in Figure 3-7, to determine whether there were detectable changes in the sensitivity of the OSLDs after each cycle as a function of accumulated dose. Batches of two nanoDot OSLDs were each exposed to doses of 1 and $10 \mathrm{~Gy}$, read in the MicroStar reader (post-irradiation readouts), then bleached for 10,120 and 600 min separately using bleaching mode (i); and finally read once more to check for the residual OSL signal after bleaching (post-bleaching readouts). Another set was bleached for 55, 600, and $2000 \mathrm{~min}$ using bleaching mode (ii), which corresponded to equivalent levels of OSL signal to the residual signal obtained after a mode (i) bleaching for 10,120 and $600 \mathrm{~min}$, respectively. The same OSLDs were re-irradiated at the same facility under the same conditions, read in the same reader and bleached again using the same bleaching unit. This irradiationreadout-bleaching-readout cycle was repeated seven times for dose fractions of 1 and 10 Gy, corresponding to total accumulated doses of 7 and $70 \mathrm{~Gy}$, respectively. 


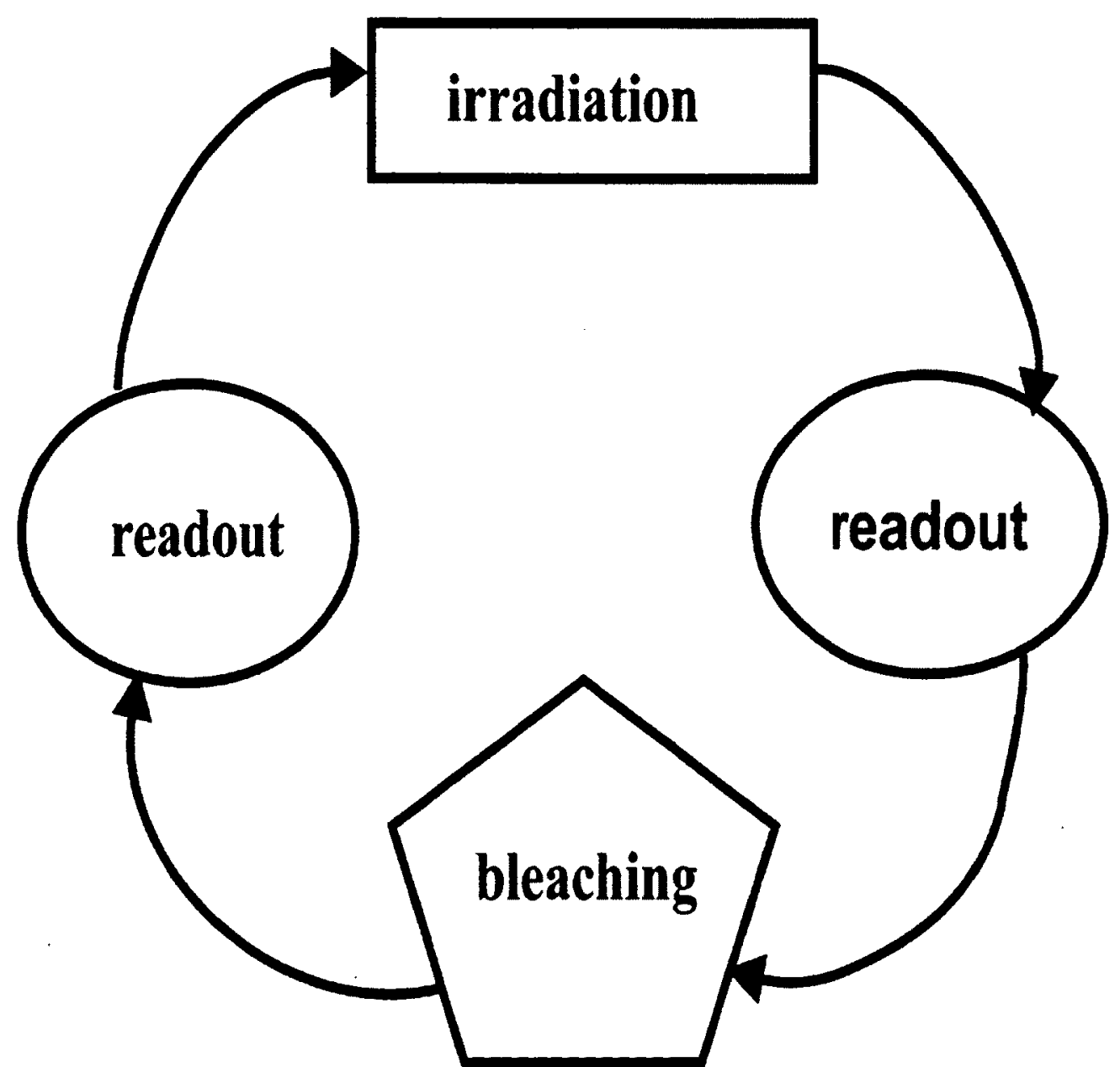

Figure 3-7: Irradiation-readout-bleaching-readout cycle performed to assess the absorbed-dose sensitivity changes of OSLDs as a function of accumulated dose.

The sensitivity changes for each nanoDot OSLD to repeated irradiation-readoutbleaching-readout cycles (Figure 3-7) were then determined, both post-irradiation and post-bleaching. It should be noted that the post-irradiation readout data for accumulated doses of 3 and $30 \mathrm{~Gy}$, were not shown because the detectors were mistakenly bleached before the post-irradiation readouts. In the plots of the sensitivity change experiments, the data points represent the average of two detectors in which each detector was read three times. To account for small dose differences in different irradiation sessions, the data were normalized to the dose measured by the ionization chamber in each irradiation session. 
The bleaching times were purposely chosen to investigate the sensitivity of the nanoDot OSLDs due to the effects of: high post-bleaching OSL signals (residual OSL signals) (10 and 55 min bleaching for bleaching modes $\mathrm{i}$ and ii, respectively); low residual OSL signals (120 and 600 min bleaching for bleaching modes $i$ and ii, respectively); and long bleaching times (600 and $2000 \mathrm{~min}$ bleaching for bleaching modes $i$ and ii, respectively). ${ }^{1}$

\subsection{Calibration protocols}

Very few publications are available on suitable calibration protocols for the use of OSLDs for radiotherapy applications in a clinical workflow. ${ }^{49,60,70}$ In Section 3.5.4, the dependence of nanoDot OSLDs on two different bleaching light wavelengths and their sensitivity to accumulated doses after multiple uses was investigated. This study seeks to investigate different calibration techniques to determine the best suitable multiple-use calibration protocol for in vivo dosimetry measurements in radiotherapy applications.

The following calibration methods were investigated in this study:

a) Batch calibration: This approach entails using a single calibration factor for all OSLDs in a batch. Using this method, the calibration curve is defined as the OSL signal $S$ versus dose $D$. Figure 3-8 shows the procedure for the method. 


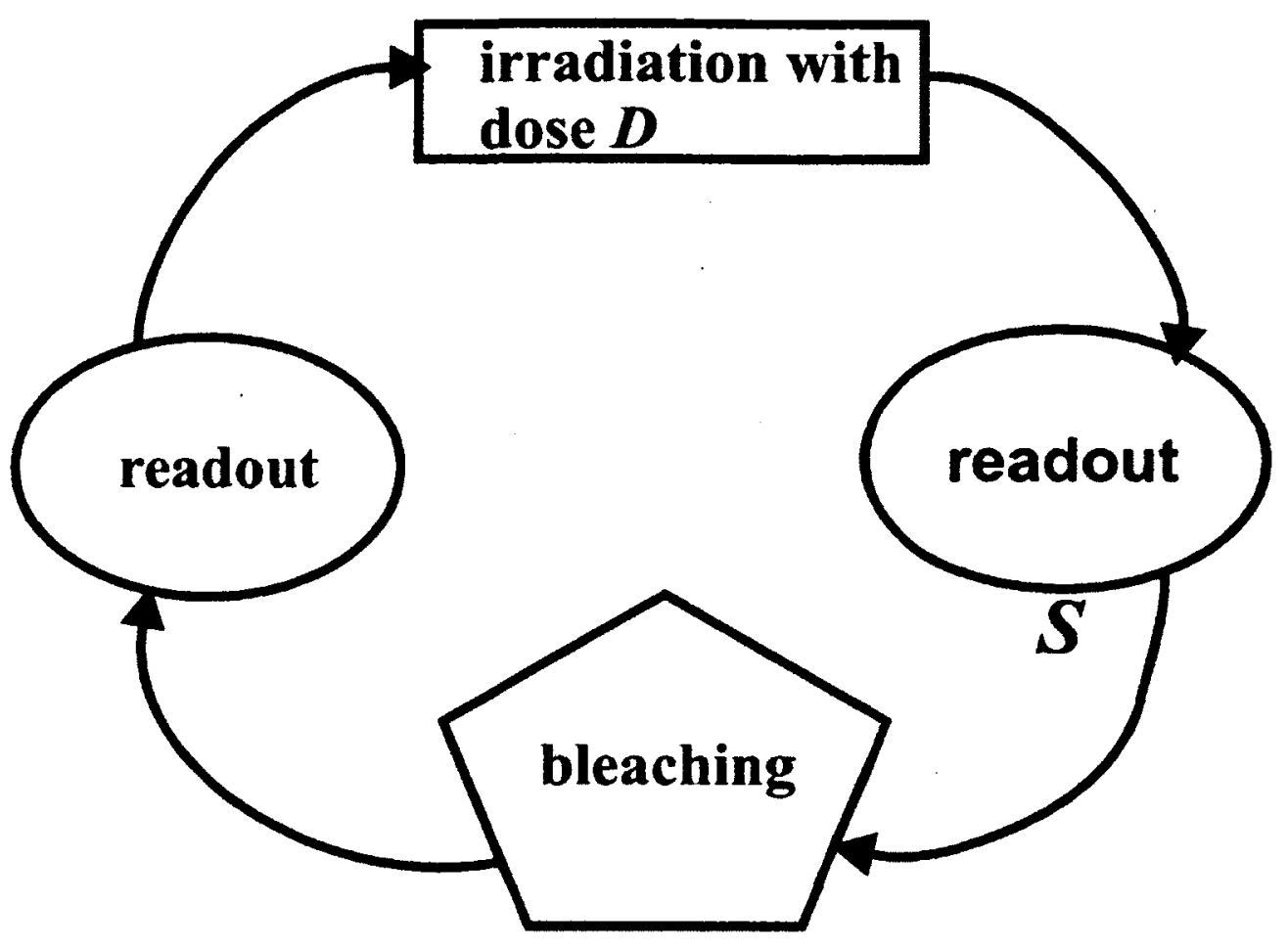

$$
M_{\text {raw }}^{\text {OSL }}=S
$$

Figure 3-8: Representation of the batch calibration method.

This calibration method is the simplest and fastest, as the raw signal from the OSLDs is used in the calibration. Hence, no extra preparation of the OSLDs is required. Jursinic, $2010^{70}$ used this method for calibration of nanoDot OSLDs.

b) Individual calibration: This approach is based on the determination of individual sensitivity factors (S.F.) for each OSLD in a batch. The individual sensitivity factors (S.F.) relates the response of each OSLD to the mean response of the batch, accounting for differences in the sensitivity of individual OSLDs. The sensitivity factor is defined as $^{33}$

$$
\text { S.F. }=\frac{n \cdot S_{i}}{\sum_{i=1}^{n} s_{i}},
$$


where $S_{i}$ represents the OSL signal of a single dosimeter from a batch of $n$ nanoDot OSLDs exposed to the same conditions. For this method, the calibration curve is defined as $S /$ S.F. versus $D$, where $S$ is OSL signal after dose $D$ as shown in Figure 3-9.

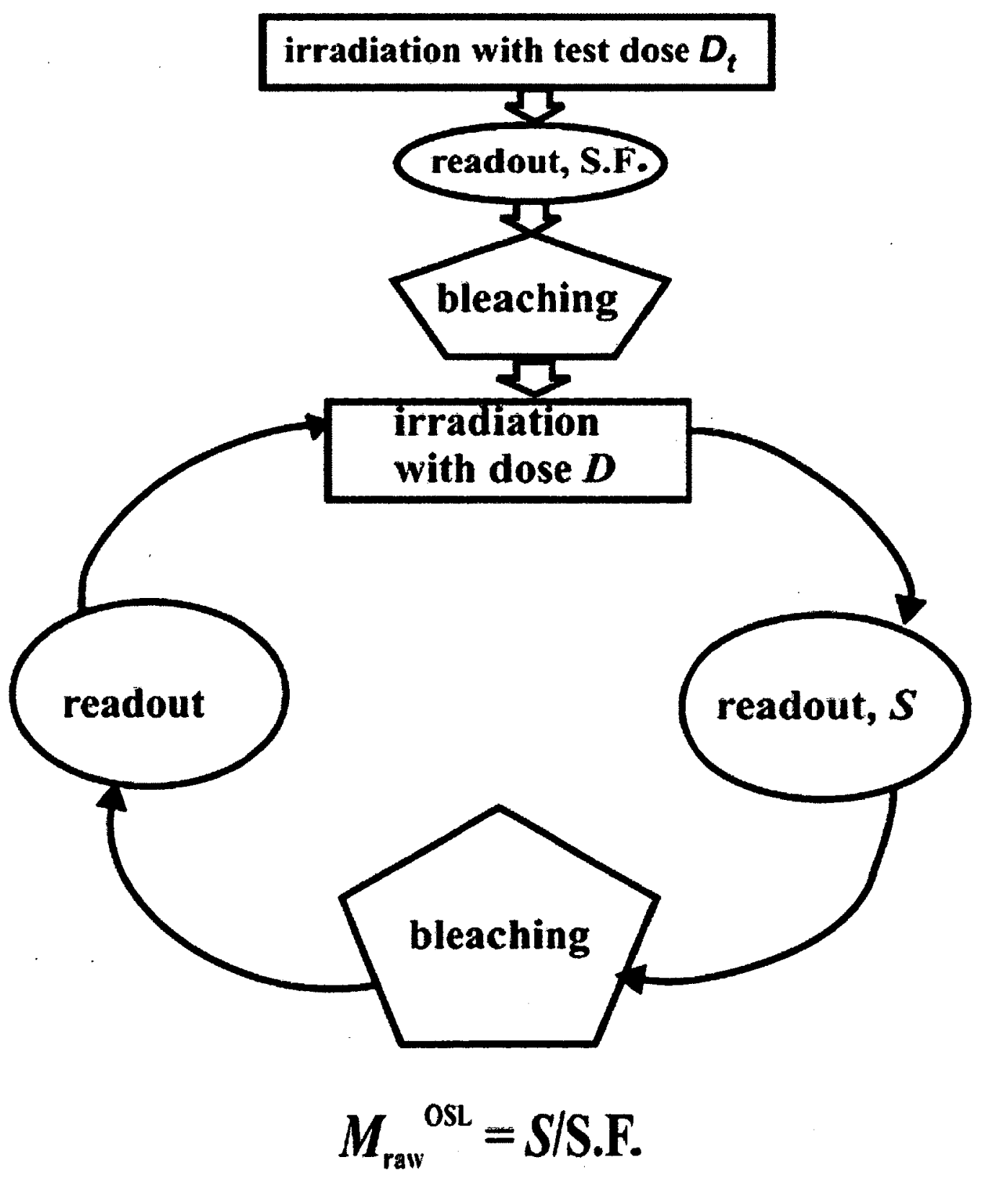

Figure 3-9: Representation of the individual calibration method. 
Sixteen new nanoDot OSLDs were selected and bleached using modes (i) and (ii), for $10 \mathrm{~min}$ and $30 \mathrm{~min}$ respectively, to remove background signals. The sensitivity factors (S.F.) were then determined by exposing the OSLDs to a small test dose, $D_{t}$, of 0.2 Gy prior to their use in the irradiation-readout-bleachingreadout cycles.

c) Reference-dose calibration: This method uses a single calibration factor and the application of a fixed reference dose, $D_{\text {ref, }}$ of $0.2 \mathrm{~Gy}$ to the OSLDs after every irradiation-readout-bleaching-readout cycle. The fixed reference dose is used to account for small changes in the sensitivity and mass of individual dosimeters in a batch. ${ }^{60}$ For this method, the calibration curve is defined as $S / S_{\text {ref }}$ versus $D$, where $S$ is the corrected OSL readout after a dose $D$ and $S_{\text {ref }}$ is the OSL readout after the fixed reference dose $D_{\text {ref; }}$ as shown in Figure 3-10.

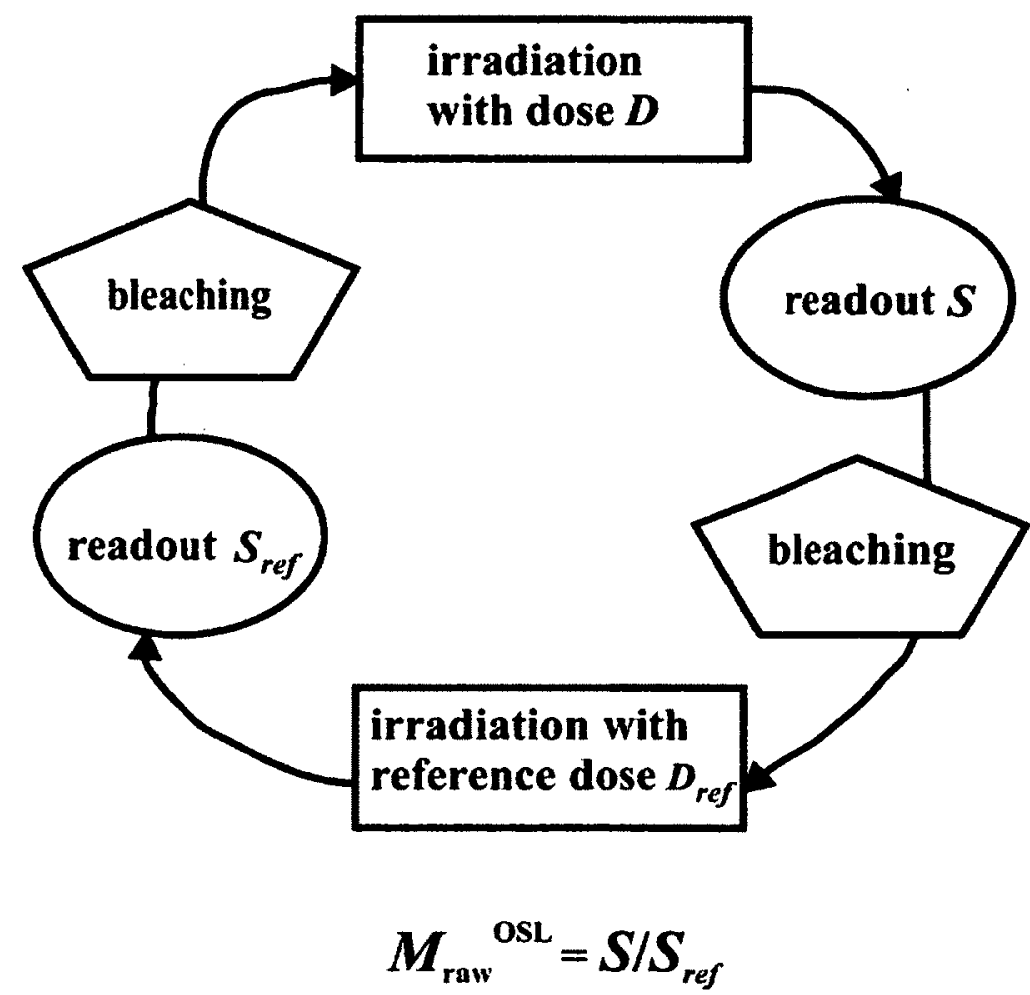

Figure 3-10: Representation of the reference-dose calibration method. 
d) Pre-dose calibration: It has been established that saturation of the deep charge traps with high doses reduces sensitivity changes of OSLDs. ${ }^{70}$ Hence, we sought to study the effects of saturating the charge traps in the nanoDot OSLDs with a very high dose, to investigate if the sensitivity changes can be reduced as a result of accumulated doses.

This approach entails saturation of the charge traps (ST, MDT and DT) in the lattice of the OSLDs with a $1 \mathrm{kGy}$ dose as was performed by Jursinic, $2010 .^{70}$ In this method, the nanoDot OSLDs are exposed to a pre-dose of $1 \mathrm{kGy}$, and then bleached for 2-3 days to low residual OSL signals before carrying out the routine irradiation-readout-bleaching-readout cycles. For this method, the calibration curve relationship follows the definitions of calibration methods a), b) and c) above.

The $1 \mathrm{kGy}$ pre-dose irradiations were performed at NRC in a cobalt- 60 therapy beam (Gammabeam X-200, Best Theratronics Ltd., Kanata, ON Canada), at water equivalent depths of $5 \mathrm{~cm}$, and backscatter of $12 \mathrm{~cm}$, with the detectors parallel to the beam axis. These irradiations were carried out at $100 \mathrm{~cm} \mathrm{SSD,} \mathrm{and}$ field size $5 \mathrm{~cm} \times 5 \mathrm{~cm}$.

For the calibration methods a), b) and c), 16 new nanoDot OSLDs in two batches of 8 , were used to investigate their sensitivity change to accumulated doses and bleaching wavelengths, for bleaching modes (i) and (ii), respectively. However, for the pre-dose calibration method d), different sets of 16 nanoDot OSLDs were used since they require a high pre-dose of $1 \mathrm{kGy}$ before use in the irradiation-readout-bleaching-readout cycles. 
For the batch and individual calibration methods a) and b), six irradiation-readoutbleaching-readout cycles were investigated, while five cycles were investigated for the reference-dose calibration method. For the pre-dose calibration, only three cycles were investigated for all dose fractions.

\subsubsection{Regeneration of OSL signals}

It has been observed that nanoDot OSLDs exposed to high doses exhibit a regeneration of the OSL signals when read after storage in the dark. ${ }^{70}$ We irradiated 16 nanoDot OSLDs to $1 \mathrm{kGy}$ in the Co-60 unit, bleached them in two sets of 8 , to very low residual OSL signals using bleaching modes (i) and (ii), respectively, then read in the MicroStar reader. The nanoDot OSLDs were then stored for different time intervals, and then read to monitor regeneration of the OSL signal.

The reading of the nanoDot OSLDs was repeated after bleaching to low residual signals following the first regeneration, to re-investigate the regeneration of the OSL signals. To quantify the regeneration of the OSL signals in dark, we fit the data to a saturation-function model: $S(t)=A[1-\exp (-t / \tau)]$ where $A$ and $\tau$ are the fit parameters. We quantify the rate of regeneration using the rise-time $t_{r}$, defined as $t_{r}=\tau \cdot \ln 9$ at different storage times.

\subsection{Dose determination and sensitivity change for different calibration protocols}

Two sets of eight nanoDot OSLDs were selected for both bleaching modes (i) and (ii), exposed to different dose fractions from $0.2-10 \mathrm{~Gy}$, and then read in irradiationreadout-bleaching-readout cycles as was done in Section 3.5.4. We then investigated the 
sensitivity change in the absorbed-dose determined for the different dose fractions using the three calibration methods a), b) and c) respectively. The selected nanoDot OSLDs were either bleached for $10 \mathrm{~h}$ with mode (i) or $40 \mathrm{~h}$ with mode (ii), to equivalent low residual OSL signals. However, the post-bleaching readout responses were not investigated.

The calibration curve for each calibration method was determined using the same two sets of eight OSLDs for both bleaching modes (i) and (ii), respectively, after exposing them to doses from 0.2 to $10 \mathrm{~Gy}$, using the OSL signal as defined by the calibration methods a), b) and c). Since supralinearity has been reported in OSLDs, ${ }^{24,33,34,73}$ the calibration curves were fit to second- and third-order polynomial models as described by:

$$
\begin{gathered}
M_{\mathrm{raw}}^{\mathrm{OSL}}(D)=b D^{2}+a D \\
\text { and } \\
M_{\mathrm{raw}}^{\mathrm{OSL}}(D)=c D^{3}+b D^{2}+a D,
\end{gathered}
$$

where $M_{\mathrm{raw}}^{\mathrm{OSL}}(D)$ is the reading as defined by the calibration method a), b) and c) and $D$ is the dose delivered to the nanoDot OSLDs as determined by the ion chamber readings, as was described in Section 3.3. It should be noted that the use of polynomial models to calibrate the nanoDot OSLDs was to fit the calibration curve to models that accurately describe the relationship of the OSL signals to dose at the dose range studied. Hence, the use of the polynomial models is for empirical purposes.

The unknown dose, $D_{\text {osL }}$ can be calculated as the positive roots of the above equations. To determine the dose delivered to each nanoDot OSLD at each cycle, the OSL reading recorded for each nanoDot is used to calculate and determine an unknown 
OSL dose DosL from the calibration fit models of Eq. (3.5) and Eq. (3.6). Using Eq. (3.5), the unknown OSL dose is determined as the positive root using the relationship:

$$
D_{\text {osL }}=\left(-a+\sqrt{a^{2}+4 b M_{\mathrm{raw}}^{\mathrm{OSL}}(D)}\right) \int_{2 b} \text {, }
$$

while for Eq. (3.6), the unknown OSL dose is determined using the relationship:

$$
\begin{aligned}
& D_{o S L}=\left(\frac{-1}{6 \sqrt[3]{2} \cdot c}\right)(1-i \sqrt{3})\left(\sqrt{\left(9 a b c-2 b^{3}+27 c^{2} M_{\mathrm{raw}}^{\mathrm{OSL}}(D)\right)^{2}+4\left(3 a c-b^{2}\right)^{3}}+\right. \\
& 9 a b c-2 b 3+27 c 2 \text { kawOSL(D) } 13+1+i 33 a c-b 23 \times 223 c 9 a b c-2 b 3+27 c 2 M \text { Kaw } \\
& \operatorname{OSL}(D) 2+43 a c-b 23+9 a b c-2 b 3+27 c 2 M \text { KawOSL(D) } 13-b 3 c .
\end{aligned}
$$

The doses were determined for all dose fractions studied and for all cycles according to the two fitting models used for the calibration curves, using Mathematica ${ }^{\mathrm{TM}}$ equation solver.

\subsubsection{Statistical analysis of sensitivity change}

To compare the doses determined by the two fitting models [Eqs. (3.7) and (3.8)] to the doses delivered, we calculated the dose difference for each dose fraction, using a chisquared relationship defined as:

$$
\chi^{2}=\sum_{i=1}^{n} \frac{\left(\frac{D_{O S L_{i}}}{D_{i}}-1\right)^{2}}{\sigma_{i}^{2}}
$$

where $D$ is the delivered dose to the OSLD; $D_{O S L}$ is the OSL dose determined by Eq. (3.7) or Eq. (3.8), for each irradiation-readout-bleaching cycle, $i$ to $n$; and $\sigma_{i}$ is the experimental standard deviation of the ratio of $D_{O S L}$ to $D$. 
To quantify the effect of increased uncertainties introduced by the different calibration methods, we used a modified weighted chi-squared relationship defined as

$$
\Gamma^{2}=\sum_{i=1}^{n}\left(\frac{D_{O S L_{i}}}{D_{i}}-1\right)^{2} \sigma_{i}^{2} .
$$

where the parameters are the same as described in Eq. (3.9).

Equations (3.9) and (3.10) can be used to determine the fitting model and calibration method that produces the most accurate dose determination and sensitivity changes respectively, for all dose fractions and cycles investigated. The lowest values of $\chi^{2}$ and $\Gamma^{2}$ represent the most accurate results. 


\section{Results}

\subsection{Dose response}

Figure 4-1 shows the dose response of the nanoDot OSLDs exposed to a dose range from 0.5 to $10 \mathrm{~Gy}$. The dotted line is a linear fit passing through the origin $\left(S_{\text {linear }}=A D\right)$ for the linear dose range from 0.5 to $1 \mathrm{~Gy}$. The dose response was fit to a linear model (blue dotted line), the second-order (red dashed line) and third-order (black solid line) polynomial models of Eq. (3.5) and (3.6) respectively.

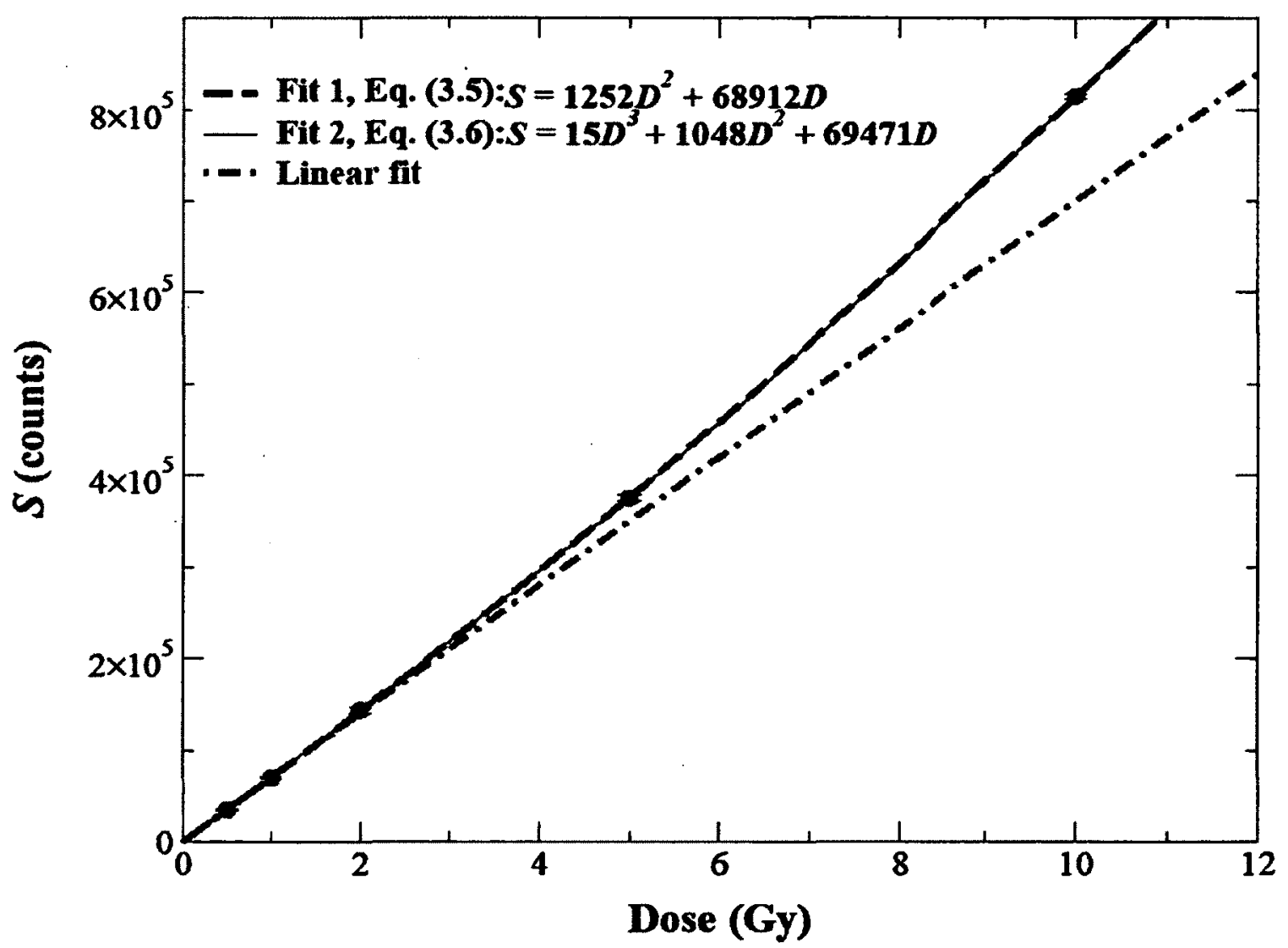

Figure 4-1: OSL signal $S$ as a function of dose for nanoDot OSLDs exposed to a range of doses from 0.5 to $10 \mathrm{~Gy}$. Each data point represents the average reading of two nanoDot OSLDs where each nanoDot was read out three times. Error bars are the standard deviation of the mean. The linear behavior (represented by the blue dotted line) was determined by fitting a linear model passing through the origin in the dose range from 0.5 to $1 \mathrm{~Gy}$. Fit 1 and Fit 2 are the second and third-order polynomial models of Eq. (3.5) and Eq. (3.6), respectively. 
It can be observed that both second- and third-order polynomial fitting models can be used to fit the dose response data. The comparison between the two fitting models was done to determine the goodness-of-fit using the Fisher's F-test and the chi-squared relationship [Eq. (3.9)]. Table 4-1 shows the values comparing the two fitting models.

Table 4-1: Parameters showing the comparison of the second- and third-order fitting models of Eq. (3.5) and Eq. (3.6) for Figure 4-1 using Fisher's F-test.

\begin{tabular}{|c|c|c|c|}
\hline Fitting Model(s) & Chi-squared value $\chi^{2}$ & F-test value & p-value \\
\hline Fit 1, Eq. (3.5) & 1014000 & \multirow{2}{*}{3.88} & 0.147 \\
\cline { 1 - 2 } Fit 2, Eq. (3.6) & 442126 & & \\
\hline
\end{tabular}

The $\chi^{2}$ value for Fit 1, with 2 degrees of freedom is about 2.3 times greater than Fit 2 with 3 degrees of freedom, while F-test value is greater than 1, implying that the third-order model [Eq. (3.6)] provides the better fit to the data. However, the p-value shows that Fit 1 provided the best fit to the data; hence the hypothesis that Fit 2 provides a better fit is rejected.

\subsection{Loss of the OSL signal as a function of sequential readouts}

Figure $4-2^{1}$ shows the loss of the OSL signal as a function of sequential readouts of nanoDot OSLDs after exposure to doses of $0.1,0.2,0.5,1$ and 10 Gy. Each data point represents the OSL signal for a single readout of each nanoDot OSLD, normalized to the first readout. Two nanoDot OSLDs were evaluated for $0.1,1$ and $10 \mathrm{~Gy}$. The rates of loss of the OSL signal for the entire dose range are shown in Table 4-2. 


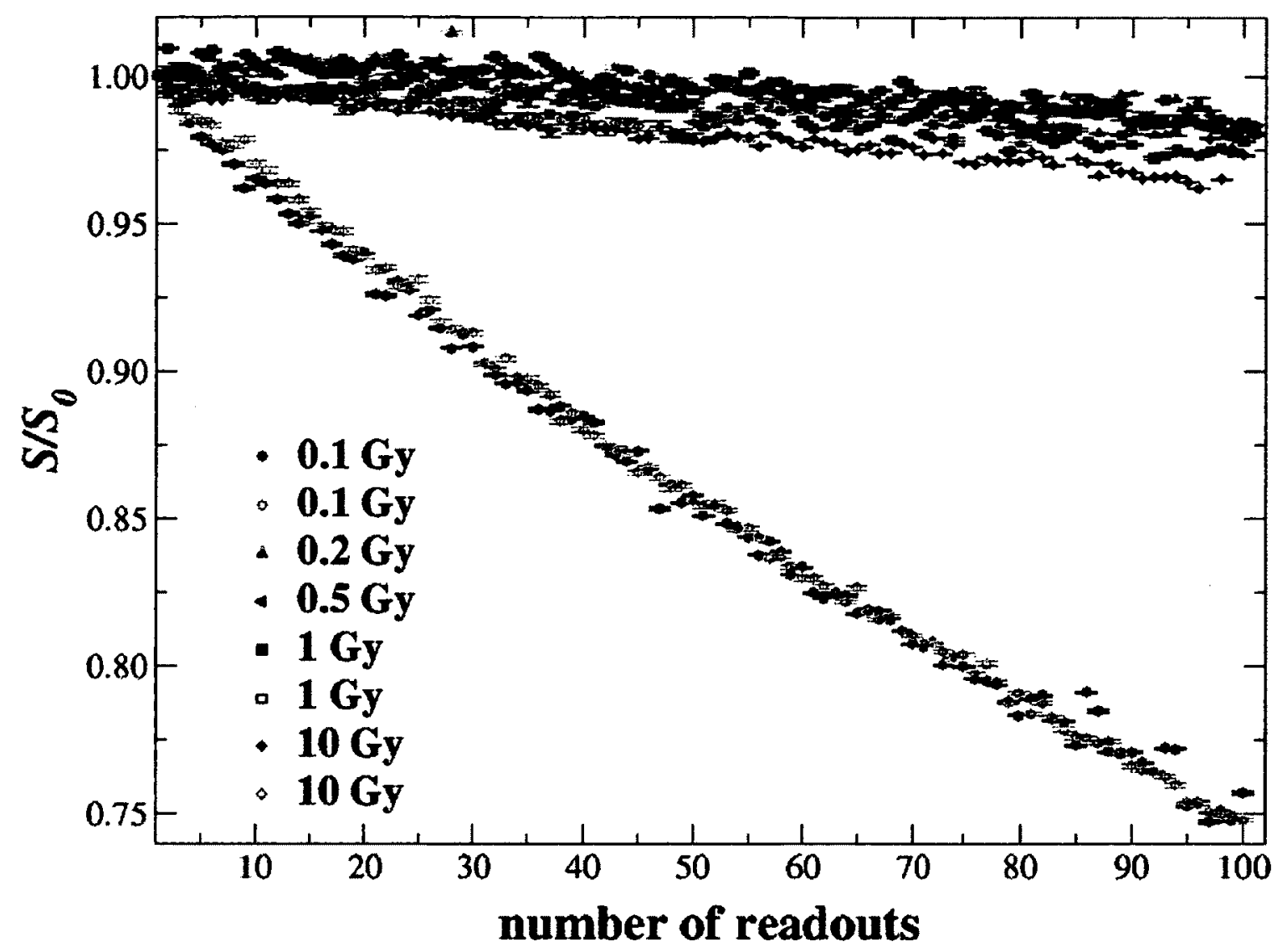

Figure 4-2: Loss of the OSL signal after successive readouts of nanoDot OSLDs exposed to doses in the range from 0.1 to $10 \mathrm{~Gy}$. Each data point represents the OSL signal $S$ of a single readout after normalization to the OSL signal of the first readout $S_{0 .}$ Error bars represent the statistical fluctuations in PMT counts.

Table 4-2: Rate of loss of OSL signal after 50 or 100 sequential readouts of nanoDot OSLDs exposed to doses from 0.1 - $10 \mathrm{~Gy}$ as shown in Figure 4-2, following a linear fit model.

\begin{tabular}{cc}
\hline Dose & Rate of loss of OSL signal $(\%)$ \\
\hline $0.1 \mathrm{~Gy}$ & $(-0.243 \pm 0.002)$ and $(-0.251 \pm 0.002)$ \\
\hline $0.2 \mathrm{~Gy}$ & $(-0.019 \pm 0.002)$ \\
\hline $0.5 \mathrm{~Gy}$ & $(-0.018 \pm 0.002)$ \\
\hline $1 \mathrm{~Gy}$ & $(-0.024 \pm 0.001)$ and $(-0.022 \pm 0.001)$ \\
\hline $10 \mathrm{~Gy}$ & $(-0.030 \pm 0.001)$ and $(-0.031 \pm 0.001)$ \\
\hline
\end{tabular}

Throughout this work we did not apply any correction for the rate of loss of the OSL signal per readout for OSLDs read using the weak-stimulation readout mode. Although we used the strong-stimulation mode to read out the residual OSL signals post- 
bleaching, we did not apply any correction factor for the loss of the OSL signal because the relative uncertainties in our residual OSL signal data are much larger than the rate of loss of the OSL signal. However, nanoDot OSLDs read using the strong-stimulation mode should be corrected for the loss of the OSL signal if one wishes to re-evaluate the dose of the detector in clinical applications. ${ }^{1}$

\subsection{Fading of the OSL signal}

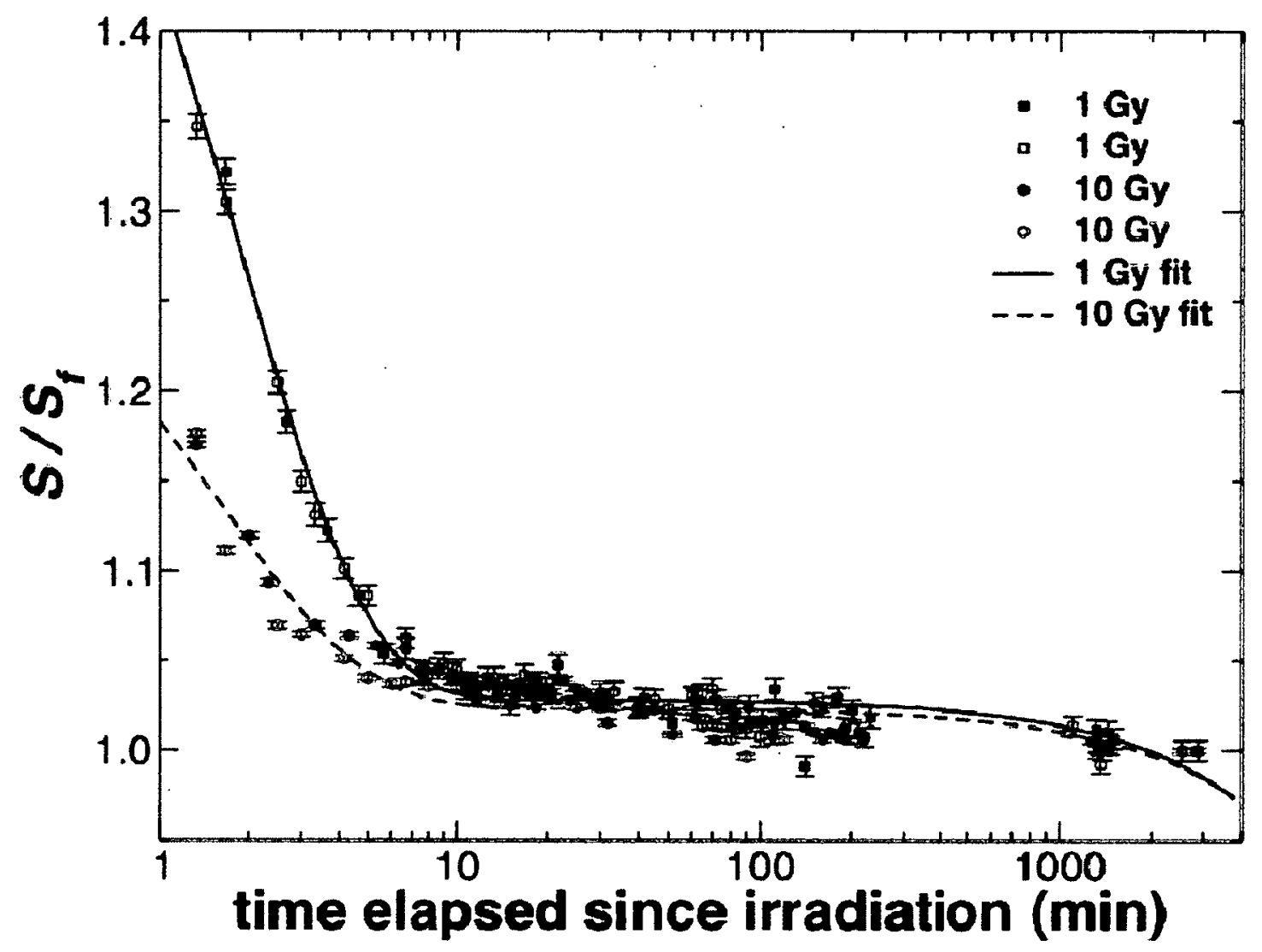

Figure 4-3: Fading of the OSL signal $S$ as a function of time elapsed since irradiation. The data sets were normalized to the last point of each respective data set ( $S_{f}$ at 2500-2800 min). Sets of two nanoDot OSLDs were exposed to doses of 1 and $10 \mathrm{~Gy}$. Each data point represents a single readout of an individual nanoDot OSLD. The error bars are the statistical fuctuation in the PMT counts. Full and dashed lines are fittings using Eq. (3.3).

Figure 4-3 ${ }^{1}$ shows the fading effect observed in two sets of two nanoDot OSLDs exposed to 1 and $10 \mathrm{~Gy}$. The data were normalized to the readout at approximately 3000 
min after irradiation. It can be seen that the fading response for the first $10 \mathrm{~min}$ post irradiation depends on the dose, as fading is more pronounced for the OSLDs exposed to $1 \mathrm{~Gy}$ compared to $10 \mathrm{~Gy}$ for the first $10 \mathrm{~min}$ interval. We obtained half-life values from the fit ranging from $0.99 \mathrm{~min}$ to $1.77 \mathrm{~min}^{1}$

Table 4-3 ${ }^{1}$ summarizes the values obtained for the fitting parameters of Eq. (3.3). Throughout all the experiments on sensitivity change, we performed all readouts at least 10 min after irradiation of the detectors.

Table 4-3: Parameters determined by fitting Eq. (3.3) to the experimental data of Figure 4-3.

\begin{tabular}{ccccc}
\hline & $\mathbf{1 ~ G y}$ & $\mathbf{1 ~ G y}$ & $\mathbf{1 0 ~ G y}$ & $\mathbf{1 0 ~ G y}$ \\
\hline $\boldsymbol{A}$ & $1.028 \pm 0.002$ & $1.029 \pm 0.002$ & $1.026 \pm 0.002$ & $1.022 \pm 0.002$ \\
\hline $\boldsymbol{B}\left(\mathbf{m i n}^{-1}\right)$ & $(-1.3 \pm 0.3) \times 10^{-5}$ & $(-1.6 \pm 0.3) \times 10^{-5}$ & $(-1.4 \pm 0.3) \times 10^{-5}$ & $(-1.2 \pm 0.3) \times 10^{-5}$ \\
\hline $\boldsymbol{C}$ & $0.72 \pm 0.06$ & $0.64 \pm 0.03$ & $0.21 \pm 0.03$ & $0.35 \pm 0.06$ \\
\hline $\boldsymbol{T}_{\mathbf{1} / 2}(\mathbf{m i n})$ & $1.26 \pm 0.08$ & $1.34 \pm 0.07$ & $1.77 \pm 0.24$ & $0.98 \pm 0.14$ \\
\hline
\end{tabular}

\subsection{Sensitivity changes}

Table $4-4^{1}$ shows the OSL signal (counts) as a function of bleaching time for OSLDs exposed to doses of 1 and $10 \mathrm{~Gy}$ and bleaching modes (i) and (ii). Different levels of residual OSL signals were obtained for different bleaching modes. Depending on the dose and bleaching time, the residual OSL signals were obtained either in the 'strong-' or 'weak-' stimulation readout modes of the reader. ${ }^{1}$ 
Table 4-4: Raw counts of the residual OSL signal after exposing the nanoDot OSLDs to 1 and $10 \mathrm{~Gy}$ and then bleaching for different periods using bleaching mode (i) (directly under the lamps) and bleaching mode (ii) (with the aid of a long-pass optical filter in front of the lamps). Except for the experiment using bleaching mode (ii) and OSLD exposed to $10 \mathrm{~Gy}$, all data were acquired for two OSLDs for each experimental condition. Values in the table are the average of three readouts for each OSLD and uncertainties are the standard deviation of the mean. Note that the readout-mode changes at 10 and $55 \mathrm{~min}$. Adapted from Omotayo et aL (2012). ${ }^{1}$

\begin{tabular}{|c|c|c|c|}
\hline \multicolumn{4}{|c|}{ Bleaching mode (i) } \\
\hline & $1 \mathrm{~Gy}$ (counts) & 10 Gy (counts) & Reader mode \\
\hline $0 \mathrm{~min}$ & $68,255 \pm 1,473$ & $813,604 \pm 25,720$ & weak-weak \\
\hline $10 \mathrm{~min}$ & $21,804 \pm 853$ & $19,720 \pm 697$ & strong-weak \\
\hline $120 \mathrm{~min}$ & $1,858 \pm 65$ & $8,737 \pm 153$ & strong-strong \\
\hline $600 \mathrm{~min}$ & $273 \pm 28$ & $999 \pm 53$ & strong-strong \\
\hline \multicolumn{4}{|c|}{ Bleaching mode (ii) } \\
\hline & $1 \mathrm{~Gy}$ (counts) & 10 Gy (counts) & Reader mode \\
\hline $0 \mathrm{~min}$ & $69,521 \pm 550$ & $789,039 \pm 11,425$ & weak-weak \\
\hline $55 \mathrm{~min}$ & $23,739 \pm 463$ & $14,063 \pm 358$ & strong-weak \\
\hline $600 \mathrm{~min}$ & $1,108 \pm 51$ & $7,687 \pm 149$ & strong-strong \\
\hline $2000 \mathrm{~min}$ & $277 \pm 29$ & $1,507 \pm 58$ & strong-strong \\
\hline
\end{tabular}

OSLDs bleached for 10 min using bleaching mode (i) had residual OSL signals of about 78 times and 70 times above the signal of an un-irradiated OSLD (background signal) for 1 and $10 \mathrm{~Gy}$, respectively. Bleaching mode (ii) removed equivalent amounts of the OSL signal for $55 \mathrm{~min}$ bleaching time. OSLDs bleached for $120 \mathrm{~min}$ using bleaching mode (i) had residual OSL signals of about 7 and 31 times above the background for 1 and $10 \mathrm{~Gy}$, respectively. Bleaching mode (ii) removed equivalent amounts of the OSL signal for $600 \mathrm{~min}$ bleaching time. Finally, OSLDs bleached for 600 min using bleaching mode (i) had residual OSL signals equivalent to background, and 4 times the background for 1 and $10 \mathrm{~Gy}$, respectively. Bleaching mode (ii) removed equivalent amounts of the OSL signal for 2000 min bleaching time. ${ }^{1}$ 


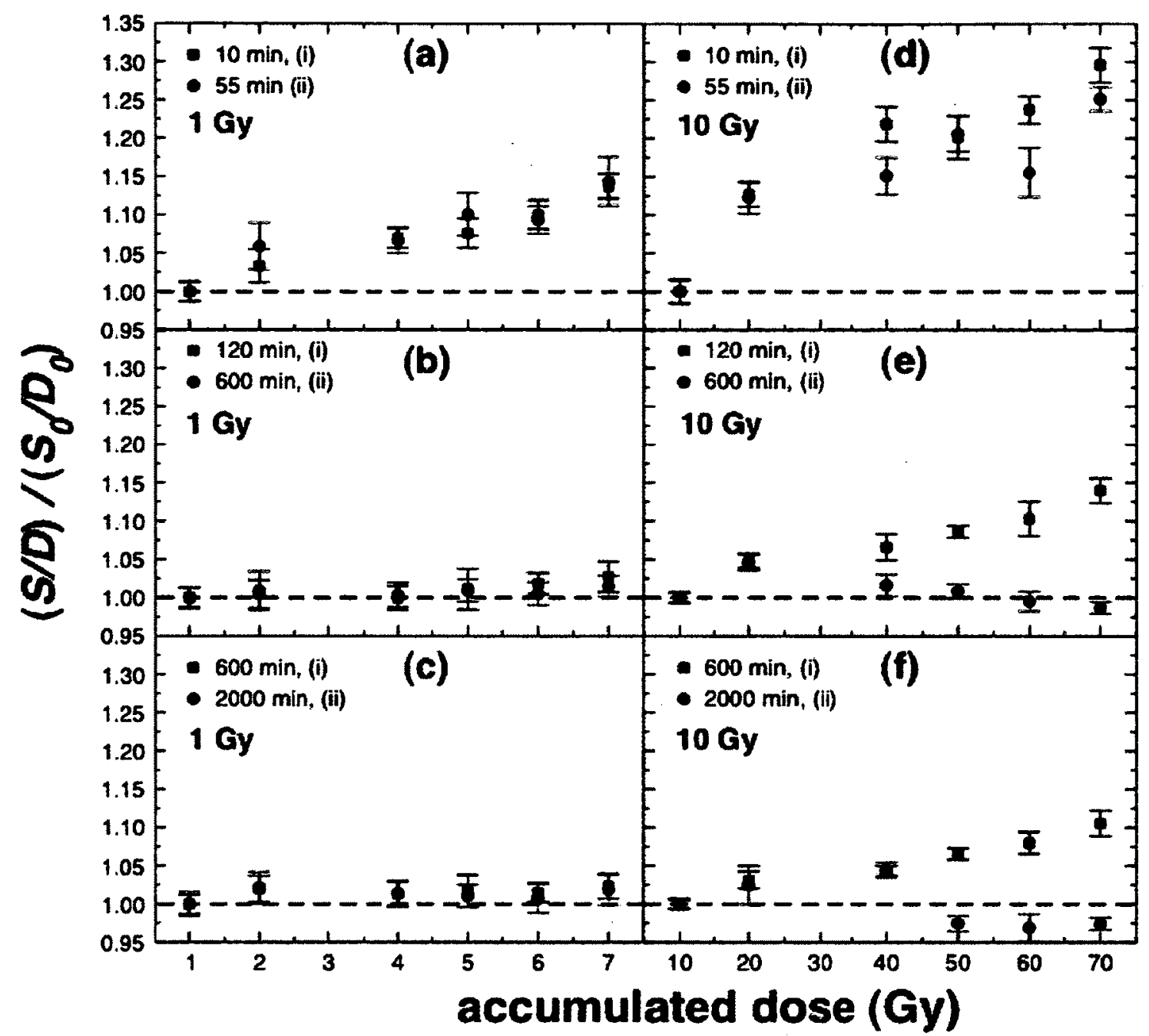

Figure 4-4: OSL signal $S$ as a function of accumulated doses from repeated cycles of irradiationreadout-bleaching-readout, after relaxation of the nanoDot OSLDs in the dark for at least $10 \mathrm{~min}$. The data were normalized to the OSL signal $S_{0}$ from the first cycle. Bleaching was performed in two modes: (i) using direct illumination of the OSLDs, and (ii) using a long-pass filter in between the light source and OSLDs. Illumination was performed for: (a) 10 and $55 \mathrm{~min}, 1 \mathrm{~Gy}$; (b) 120 and $600 \mathrm{~min}, 1$ Gy; (c) 600 and $200 \mathrm{~min}, 1 \mathrm{~Gy}$; (d) 10 and $5 \mathrm{~min}, 10 \mathrm{~Gy}$; (e) 120 and $600 \mathrm{~min}, 10 \mathrm{~Gy}$; and (f) 600 and $2000 \mathrm{~min}, 10 \mathrm{~Gy}$. Each data point represents the average of three readouts (same OSLD), with error bars representing the standard deviation of the mean for the three readouts. Adapted from Omotayo et al. (2012).

The change in the sensitivity of nanoDot OSLDs as a function of irradiationreadout-bleaching-readout cycles was determined by plotting the OSL signal as a function of the accumulated dose. Figure $4-4$ shows the post-irradiation readouts as a function of accumulated doses. For an accumulated dose of $7 \mathrm{~Gy}$ (1 Gy fractions), the sensitivity of the OSLDs increased $14 \%$ for modes (i) (10 min bleaching time) and (ii) 
(55 min bleaching time) (Figure 4-4a). For an accumulated dose of 70 Gy (10 Gy fractions), the sensitivity increased $30 \%$ and $25 \%$ for modes (i) (10 min) and (ii) (55 $\min$ ), respectively (Figure $4-4 d) .{ }^{1}$

Figure 4-4b and Figure 4-4e show the post-irradiation readouts of nanoDot OSLDs as a function of accumulated doses for bleaching times of 120 and 600 min using modes (i) and (ii), respectively. For an accumulated dose of $7 \mathrm{~Gy}$ (1 Gy fractions), the sensitivity of the OSLDs increased $2.7 \%$ and $1.5 \%$ for modes (i) (120 min) and (ii) (600 $\mathrm{min})$, respectively (Figure 4-4b). For an accumulated dose of $70 \mathrm{~Gy}$ (10 Gy fractions), the sensitivity increased 14\% for mode (i) (120 min) and decreased 1.3\% for mode (ii) (600 $\min$ ) (Figure 4-4e). For mode (ii) and accumulated dose of $20 \mathrm{~Gy}$, an increase of $4.6 \%$ was observed in the sensitivity of the OSLDs. ${ }^{1}$

Figure 4-4c and Figure 4-4f show the post-irradiation readouts of nanoDot OSLDs as a function of accumulated doses for bleaching times of 600 and $2000 \mathrm{~min}$ using modes (i) and (ii), respectively. For an accumulated dose of 7 Gy (1 Gy fractions), the sensitivity increased $2.3 \%$ and $1.8 \%$ for modes (i) (600 $\mathrm{min}$ ) and (ii) (2000 min), respectively. For an accumulated dose of $70 \mathrm{~Gy}$ (10 Gy fractions), the sensitivity increased 10\% for mode (i) (600 $\mathrm{min}$ ) and decreased $2.5 \%$ for mode (ii) (2000 min). These trends are similar to the trends obtained for the 120 and 600 min bleaching times (Figure 4-4b and Figure 4-4e). ${ }^{1}$ 


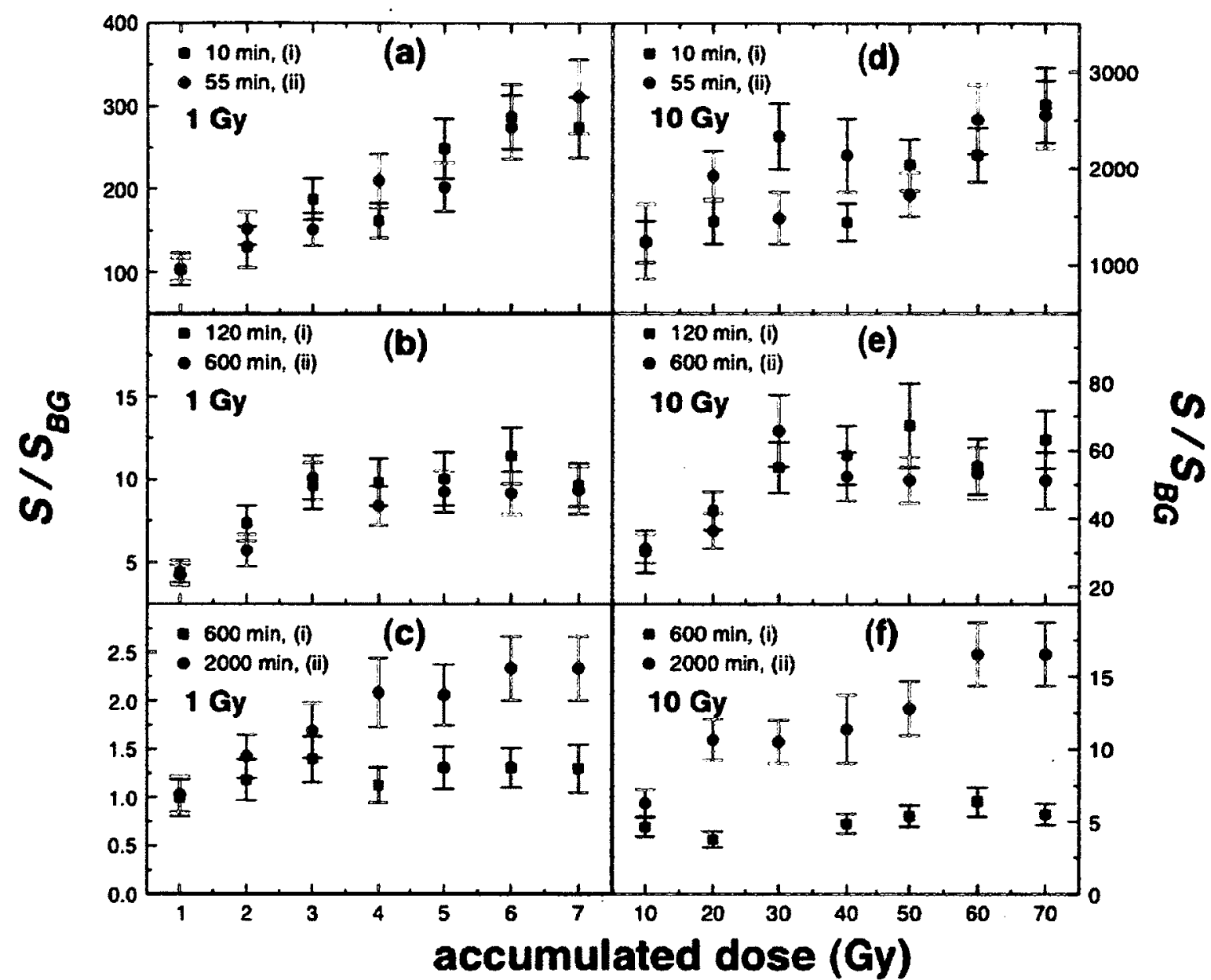

Figure 4-5: Post-bleaching OSL signal $S$ as a function of accumulated doses from repeated cycles of irradiation-readout-bleaching-readout. The data were normalized to $S_{B G}=(371 \pm 48)$ counts, which is the average of the residual signal of detectors exposed to an accumulated dose of $1 \mathrm{~Gy}$ and bleached for 600 min and 2000 min using bleaching modes (i) and (ii), respectively. The caption of Figure 4-4 provides the details of this plot. Adapted from Omotayo et aL (2012).

Figure 4-5 shows the post-bleaching readouts of the residual signals for the same twenty-four nanoDot OSLDs used to obtain the data from Figure 4-4. The data in Figure 4-5 were normalized to the average of the residual OSL signal of detectors exposed to an accumulated dose of $1 \mathrm{~Gy}$ and bleached for 600 and 2000 min using bleaching modes (i) and (ii), respectively. The normalization factor was determined to be $(371 \pm 48)$ PMT counts, which can be assumed to be the background PMT counts of an un-irradiated detector. For an accumulated dose of $7 \mathrm{~Gy}$ (1 Gy fractions) and bleaching times of 10 
min (mode i) and 55 min (mode ii), the residual OSL signals were about 300 times higher than the signal of an un-irradiated OSLD. For an accumulated dose of $70 \mathrm{~Gy}$ (10 Gy fractions) and bleaching times of $10 \mathrm{~min}$ (mode i) and $55 \mathrm{~min}$ (mode ii), the residual OSL signals were about 2500 times higher than the signal of an un-irradiated OSLD (Figure 4-5a and Figure 4-5d). Note that the residual OSL signals of Figure 4-5d for OSLDs exposed to $10 \mathrm{~Gy}$ fractions and bleached for 10 and $55 \mathrm{~min}$ using bleaching modes (i) and (ii), respectively, were read out in the weak-stimulation mode while all the other readouts of the residual OSL signals were performed using the strong-stimulation mode. Thus, to compare the results from different stimulation modes we multiplied the data acquired using the weak-stimulation mode by a scaling factor. The scaling factor was calculated using the calibration data for the weak- and strong-stimulation modes as the ratio of the slopes of calibration curves. This scaling factor may be used to qualitatively compare results from the two stimulation modes. However, it may not be used for quantitative comparison until further investigations are performed to determine its validity. ${ }^{1}$

Figure 4-5b and Figure 4-5e show the post-bleaching residual OSL signals after bleaching for $120 \mathrm{~min}$ (mode i) and $600 \mathrm{~min}$ (mode ii). For an accumulated dose of $7 \mathrm{~Gy}$ (1 Gy fractions) and $70 \mathrm{~Gy}$ (10 Gy fractions), the residual OSL signals for both bleaching modes were 10 and 60 times higher than the signal of an un-irradiated OSLD, respectively. ${ }^{1}$

Figure 4-5c and Figure 4-5f show the post-bleaching residual signals after 600 and 2000 min bleaching, for modes (i) and (ii). For an accumulated dose of 7 Gy (1 Gy fractions), the residual OSL signals were 1.3 and 2.3 times higher than the signal of an un-irradiated OSLD for bleaching modes (i) (600 min) and (ii) (2000 min), respectively. 
For an accumulated dose of $70 \mathrm{~Gy}$ (10 Gy fractions), the residual OSL signals were 5 and 16 times higher than the signal of an un-irradiated OSLD for bleaching modes (i) (600 min) and (ii) (2000 $\mathrm{min})$, respectively. ${ }^{1}$

\subsection{Calibration protocols}

\subsubsection{Batch calibration}

\subsubsection{Calibration curve}

Figure 4-6 shows the calibration plot for the sixteen nanoDot OSLDs after exposure to doses from 0.2 to 10 Gy using the batch calibration method.

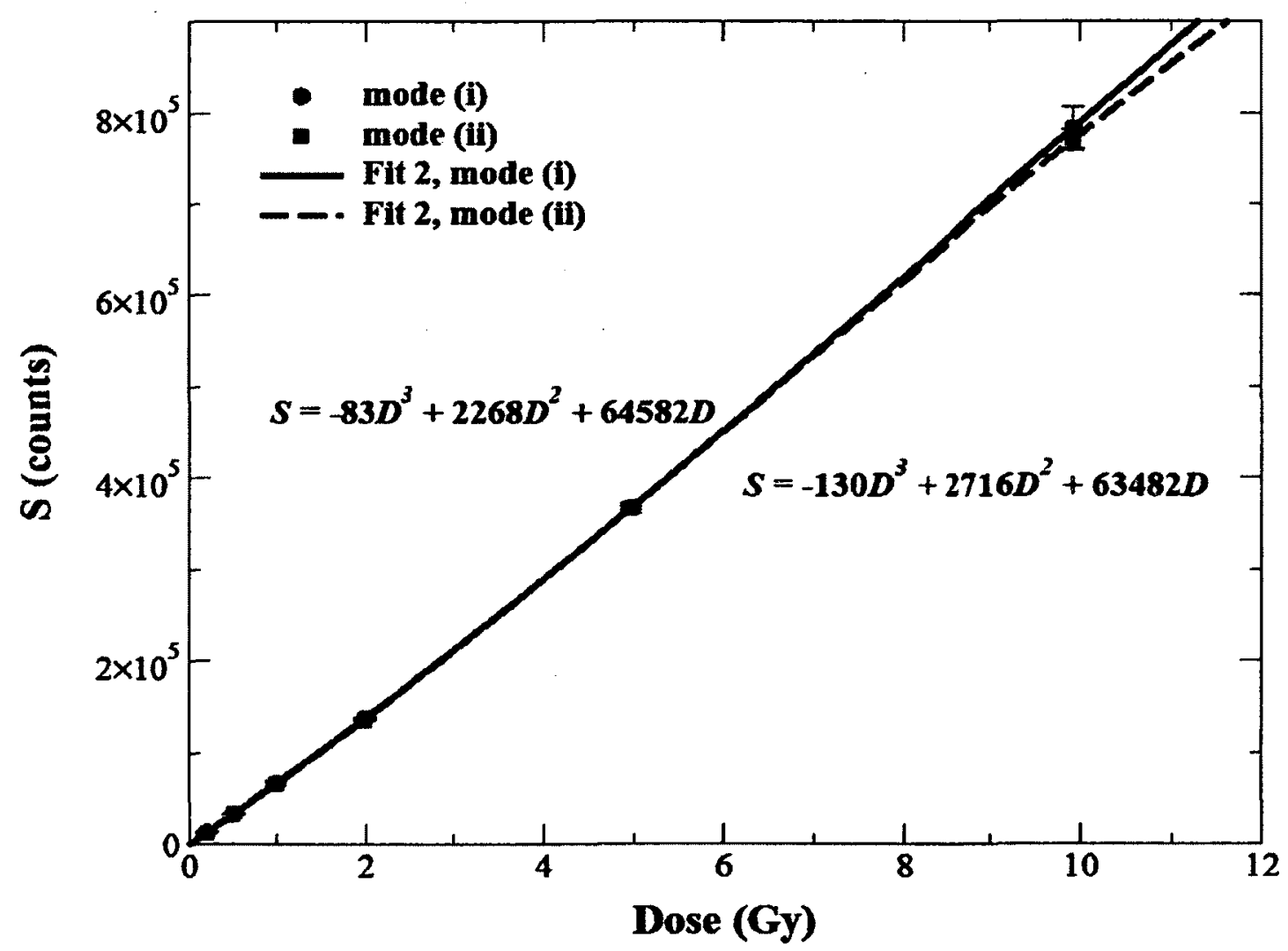

Figure 4-6: OSL signal $S$ as a function of dose for nanoDot OSLDs exposed to doses $D$ from 0.5 to 10 Gy. Each data point represents the average reading of three readouts of one nanoDot OSLD, except at 1 and $10 \mathrm{~Gy}$ where two OSLDs were used. Error bars are the standard deviation of the mean. The fit was determined using the model described by Eq. (3.6). 
The data were fit to the third-order model of Eq. (3.6) [Fit 2]. The second-order model of Eq. (3.5) [Fit 1] is not shown here as we determined it underestimated the unknown dose estimations $D_{O S L}$, at doses below $1 \mathrm{~Gy}$.

\subsubsection{Absorbed-dose determination}

Figure 4-7 shows the absorbed-dose determination using the batch calibration method, for the same nanoDot OSLDs used in Figure 4-6. Each data point represents the ratio of the OSLD measured dose $D_{\text {OSL, }}$, determined by the solution to the calibration fitting models from Eq. (3.7) [Fit 1] and Eq. (3.8) [Fit 2], to the actual dose $D$ delivered per cycle to the nanoDot OSLDs, as measured by the ion chamber. Thus, deviations from unity imply that the OSLD measured dose, $D_{O S L}$, do not agree with the delivered dose $D$. It should be clearly stated and noted that the actual dose delivered to the OSLDs at cycle 3 for all dose fractions in Figure 4-7, was significantly lower than the intended nominal dose. The OSLDs may have been exposed to different irradiation conditions compared to the other cycles; hence, a lower OSLD measured dose was determined. Thus, throughout this work, data from cycle 3 are not included in our analysis.

It can be observed that Fit 2 - obtained using Eq. (3.8) - provided the best dose estimates $( \pm 3.5 \%)$ across all dose fractions regardless of the bleaching mode, except for 0.2 Gy dose fractions, where $4-6 \%$ differences were observed. For 0.2 Gy fractions, Fit 1 - obtained using Eq. (3.7) - provided dose estimates within $4.7 \%$ for nanoDot OSLDs bleached using mode (ii). For nanoDot OSLDs bleached using filter mode (i), Fit 2 provided the best absorbed-dose estimates across all dose fractions except at accumulated doses greater than $40 \mathrm{~Gy}$ where $5-5.6 \%$ absorbed-dose changes were observed. 

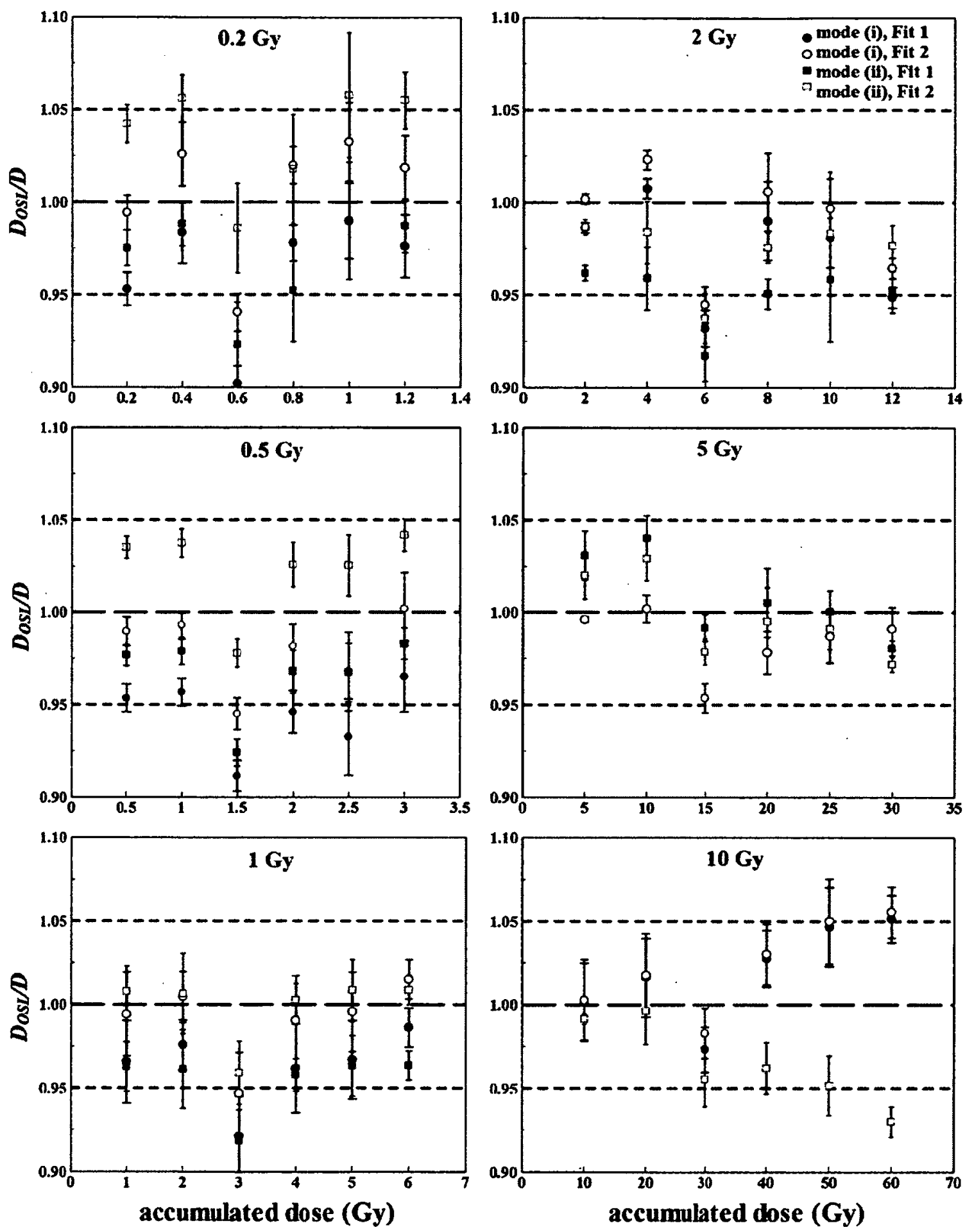

Figure 4-7: OSLD measured dose $D_{\text {osL }}$ divided by the delivered dose $D$, as a function of accumulated doses for batch calibration method. Bleaching of the nanoDot OSLDs was $10 \mathrm{~h}$ for mode (i) and $40 \mathrm{~h}$ for mode (ii) for all dose fractions. Each data point represents the ratio of the OSLD measured dose $D_{\text {ost }}$, determined by the calibration curves using fitting models from Eq. (3.7) [Fit 1] and Eq. (3.8) [Fit 2], to the dose $D$ delivered per cycle to the nanoDot OSLDs, as measured by the ion chamber.

Short dashed lines represent the $\pm \mathbf{5 \%}$ interval, from the expected unity value (long dash). 
Table 4-5 shows the values of the chi-squared and modified chi-squared relationships for all dose fractions, comparing both absorbed-dose determination using Fit 1 and Fit 2, for the batch calibration method. Fit 2 produced the lowest values for $\chi^{2}$ and $\Gamma^{2}$ for bleaching mode (i), except at 5 and $10 \mathrm{~Gy}$, where Fit 1 produced lower values. For bleaching mode (ii), Fit 1 produced slightly lower values for both $\chi^{2}$ and $\Gamma^{2}$ at 0.2 Gy and 0.5 Gy dose fractions, meaning Fit 1 produced the best absorbed-dose determination variation.

The goodness of fit test p-value for the two fitting functions [see Table 4-5] showed that the second-order polynomial fitting model (Fit 1) provided the best fit for the calibration. However, we observed that the third-order model (Fit 2) produced the best estimations of OSL unknown dose $D_{O S L}$ at dose fractions from $1 \mathrm{~Gy}$ to $10 \mathrm{~Gy}$, which are the most relevant in radiotherapy treatments and applications.

Table 4-5: Values of the $\chi^{2}$ and $\Gamma^{2}$ statistics described by Eqs. (3.9) and (3.10), comparing the two fitting models for dose estimations in Figure 4-7, for bleaching modes (i) and (ii), respectively.

\begin{tabular}{|c|c|c|c|c|}
\hline \multicolumn{5}{|c|}{ Bleaching mode (i) } \\
\hline & \multicolumn{2}{|c|}{$x^{2}$} & \multicolumn{2}{|c|}{$\Gamma^{2}$} \\
\hline Dose fractions & Fit 1 & Fit 2 & Fit 1 & Fit 2 \\
\hline $0.2 \mathrm{~Gy}$ & 35.79 & 10.03 & $5.034 \times 10^{-7}$ & $8.234 \times 10^{-7}$ \\
\hline $0.5 \mathrm{~Gy}$ & 108.36 & 7.32 & $3.008 \times 10^{-6}$ & $5.462 \times 10^{-7}$ \\
\hline $1 \mathrm{~Gy}$ & 10.05 & 1.94 & $2.502 \times 10^{-6}$ & $1.361 \times 10^{-7}$ \\
\hline $2 \mathrm{~Gy}$ & 112.52 & 59.92 & $2.266 \times 10^{-7}$ & $7.175 \times 10^{-8}$ \\
\hline $5 \mathrm{~Gy}$ & 6.31 & 13.76 & $5.054 \times 10^{-8}$ & $1.082 \times 10^{-7}$ \\
\hline $10 \mathrm{~Gy}$ & 20.16 & 20.19 & $2.105 \times 10^{-6}$ & $2.813 \times 10^{-6}$ \\
\hline \multicolumn{5}{|c|}{ Bleaching mode (ii) } \\
\hline & \multicolumn{2}{|c|}{$x^{2}$} & \multicolumn{2}{|c|}{$\Gamma^{2}$} \\
\hline Dose fractions & Fit 1 & Fit 2 & Fit 1 & Fit 2 \\
\hline $0.2 \mathrm{~Gy}$ & 11.43 & 52.96 & $1.975 \times 10^{-6}$ & $5.489 \times 10^{-6}$ \\
\hline $0.5 \mathrm{~Gy}$ & 40.51 & 87.66 & $4.666 \times 10^{-7}$ & $5.311 \times 10^{-7}$ \\
\hline $1 \mathrm{~Gy}$ & 49.49 & 1.58 & $1.833 \times 10^{-6}$ & $6.991 \times 10^{-8}$ \\
\hline $2 \mathrm{~Gy}$ & 146.54 & 25.25 & $2.945 \times 10^{-6}$ & $4.972 \times 10^{-7}$ \\
\hline $5 \mathrm{~Gy}$ & 38.94 & 60.02 & $4.207 \times 10^{-7}$ & $2.094 \times 10^{-7}$ \\
\hline $10 \mathrm{~Gy}$ & 85.97 & 80.28 & $1.036 \times 10^{-6}$ & $1.477 \times 10^{-6}$ \\
\hline
\end{tabular}




\subsubsection{Individual calibration}

\subsubsection{Sensitivity factors (S.F.)}

Table 4-6 and Table 4-7 show the sensitivity factors (S.F.) defined by Eq. (3.4) for the sixteen nanoDot OSLDs bleached with mode (i) and mode (ii) respectively.

Table 4-6: Sensitivity factors for 16 nanoDot OSLDs after exposure to test dose of $20 \mathrm{cGy}$. The OSLDs were bleached with mode (i) for 10 min before exposure to remove background signals.

\begin{tabular}{|c|c|}
\hline Dosimeter \# & Sensitivity factors \\
\hline DN09441276U & $0.999 \pm 0.010$ \\
\hline DN09431954O & $0.976 \pm 0.019$ \\
\hline DN09434579H & $1.006 \pm 0.011$ \\
\hline DN094352340 & $1.048 \pm 0.014$ \\
\hline DN09436174X & $0.985 \pm 0.012$ \\
\hline DN09438881M & $1.001 \pm 0.017$ \\
\hline DN09432995H & $1.017 \pm 0.017$ \\
\hline DN09432649K & $1.023 \pm 0.018$ \\
\hline DN09434308U & $1.001 \pm 0.012$ \\
\hline DN09434923Q & $0.978 \pm 0.014$ \\
\hline DN09440228W & $0.967 \pm 0.011$ \\
\hline DN094327400 & $0.997 \pm 0.014$ \\
\hline DN09432643W & $1.012 \pm 0.012$ \\
\hline DN09431643X & $1.005 \pm 0.019$ \\
\hline DN09441138W & $0.989 \pm 0.012$ \\
\hline DN094352712 & $0.995 \pm 0.013$ \\
\hline
\end{tabular}

Figure 4-8 and Figure 4-9 shows plots of the sensitivity factors for the nanoDot OSLDs after exposure to a $20 \mathrm{cGy}$ test dose subsequent to bleaching with mode (i) and (ii), for 10 and $30 \mathrm{~min}$, respectively. Error bars represent the standard deviation for the average readout of the nanoDot OSLDs. It can be observed that nanoDot OSLDs from the same batch have different sensitivity factors (S.F.) even after exposure to the same dose and conditions. The S.F. values were all within $3.0 \%$ regardless of the bleaching mode, except for two OSLDs (DN09440228W, DN094352340) which were within 3.3\% and $4.8 \%$ from unity, respectively. This shows that the nanoDot OSLDs from the same batch are reproducible within 3\%. 
Table 4-7: Sensitivity factors for 16 nanoDot OSLDs after exposure to test dose of $20 \mathrm{cGy}$. The OSLDs were bleached with mode (ii) for $30 \mathrm{~min}$ before exposure to remove background signals.

\begin{tabular}{|c|c|}
\hline Dosimeter \# & Sensitivity factors \\
\hline DN09431936M & $1.004 \pm 0.013$ \\
\hline DN09432719L & $1.011 \pm 0.019$ \\
\hline DN09435273Y & $1.011 \pm 0.016$ \\
\hline DN09439902P & $1.014 \pm 0.019$ \\
\hline DN09432759H & $1.011 \pm 0.012$ \\
\hline DN094345212 & $1.013 \pm 0.013$ \\
\hline DN094353116 & $0.988 \pm 0.009$ \\
\hline DN09440112B & $0.984 \pm 0.009$ \\
\hline DN09431968F & $0.979 \pm 0.018$ \\
\hline DN094411328 & $1.019 \pm 0.017$ \\
\hline DN09433844P & $0.999 \pm 0.014$ \\
\hline DN09437452Y & $1.009 \pm 0.011$ \\
\hline DN09430676P & $0.994 \pm 0.015$ \\
\hline DN09433882P & $0.981 \pm 0.011$ \\
\hline DN09434908I & $0.999 \pm 0.013$ \\
\hline DN09433946J & $0.982 \pm 0.016$ \\
\hline
\end{tabular}

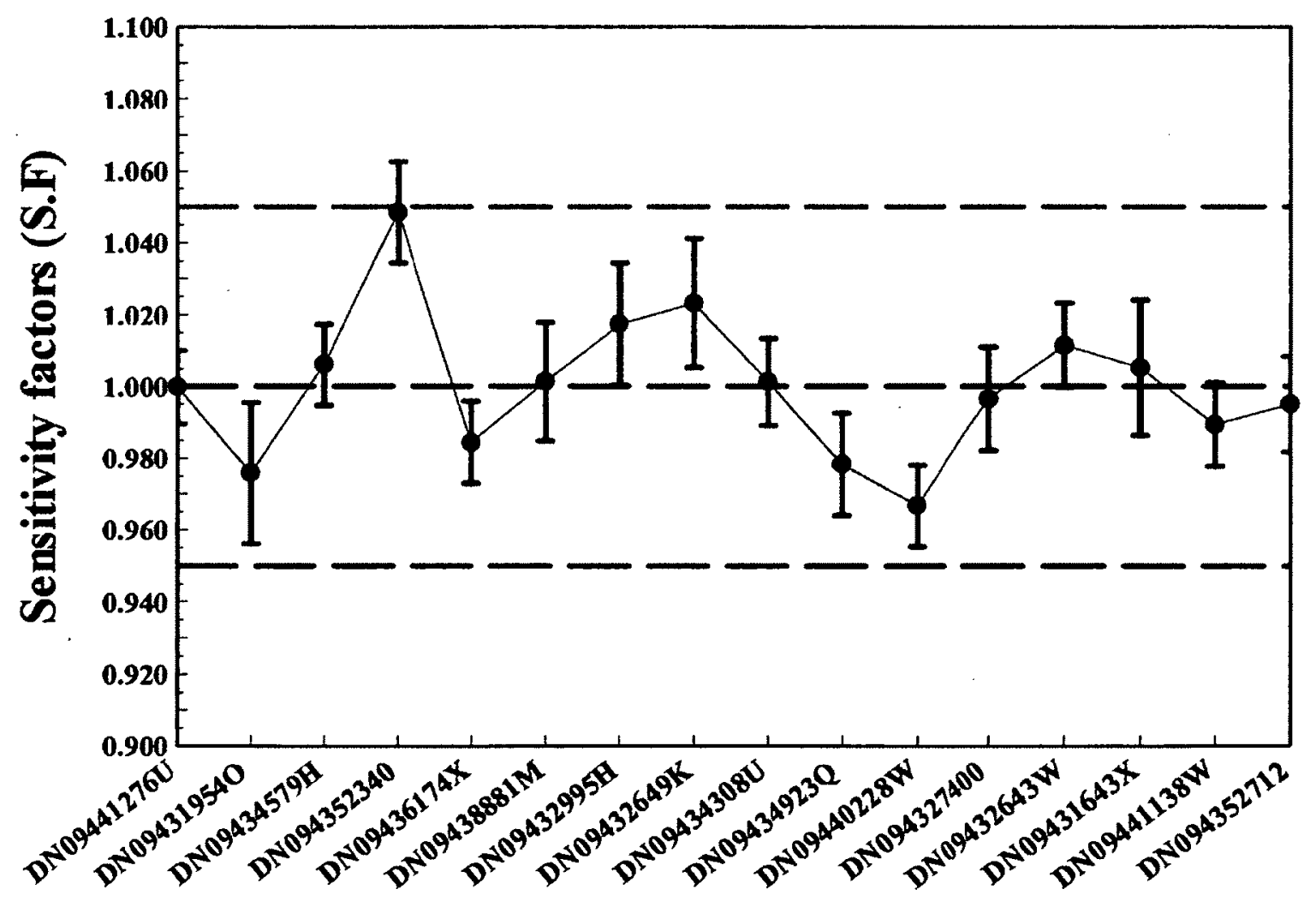

Figure 4-8: Sensitivity factors for nanoDot OSLDs exposed to a test dose of $20 \mathrm{cGy}$, after bleaching with mode (i) for 10 min to remove background signals. S.F. values are determined by Eq. (3.4).

Dashed lines represent the $5 \%$ interval from the average S.F for all the OSLDs. 


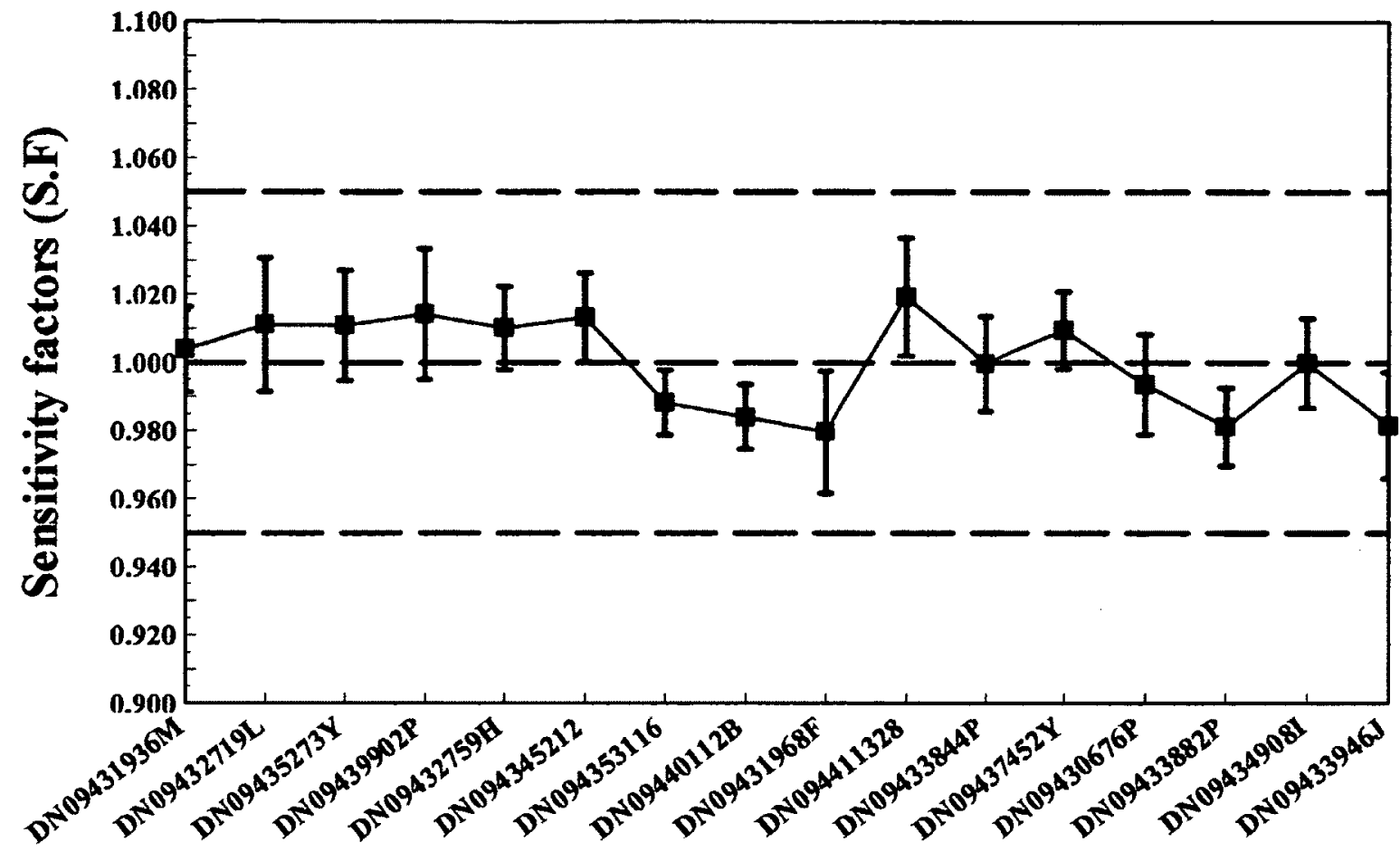

Figure 4-9: Sensitivity factors for nanoDot OSLDs exposed to a test dose of $20 \mathrm{cGy}$, after bleaching with mode (ii) for $30 \mathrm{~min}$ to remove background signals. S.F. values are determined by Eq. (3.4).

Dashed lines represent the $5 \%$ interval from the average S.F for all the OSLDs.

\subsubsection{Calibration curve}

Figure 4-10 shows the calibration plot for the sixteen nanoDot OSLDs in Figure 4-6, after exposure to doses from 0.2 to $10 \mathrm{~Gy}$, using the individual calibration method. The dose response data were fit to the third-order model of Eq. (3.6) [Fit 2]. The fit to the secondorder model of Eq. (3.5) [Fit 1] is not shown here as we determined it underestimated the unknown dose estimations $D_{\text {OSL, }}$ at doses below $1 \mathrm{~Gy}$. 


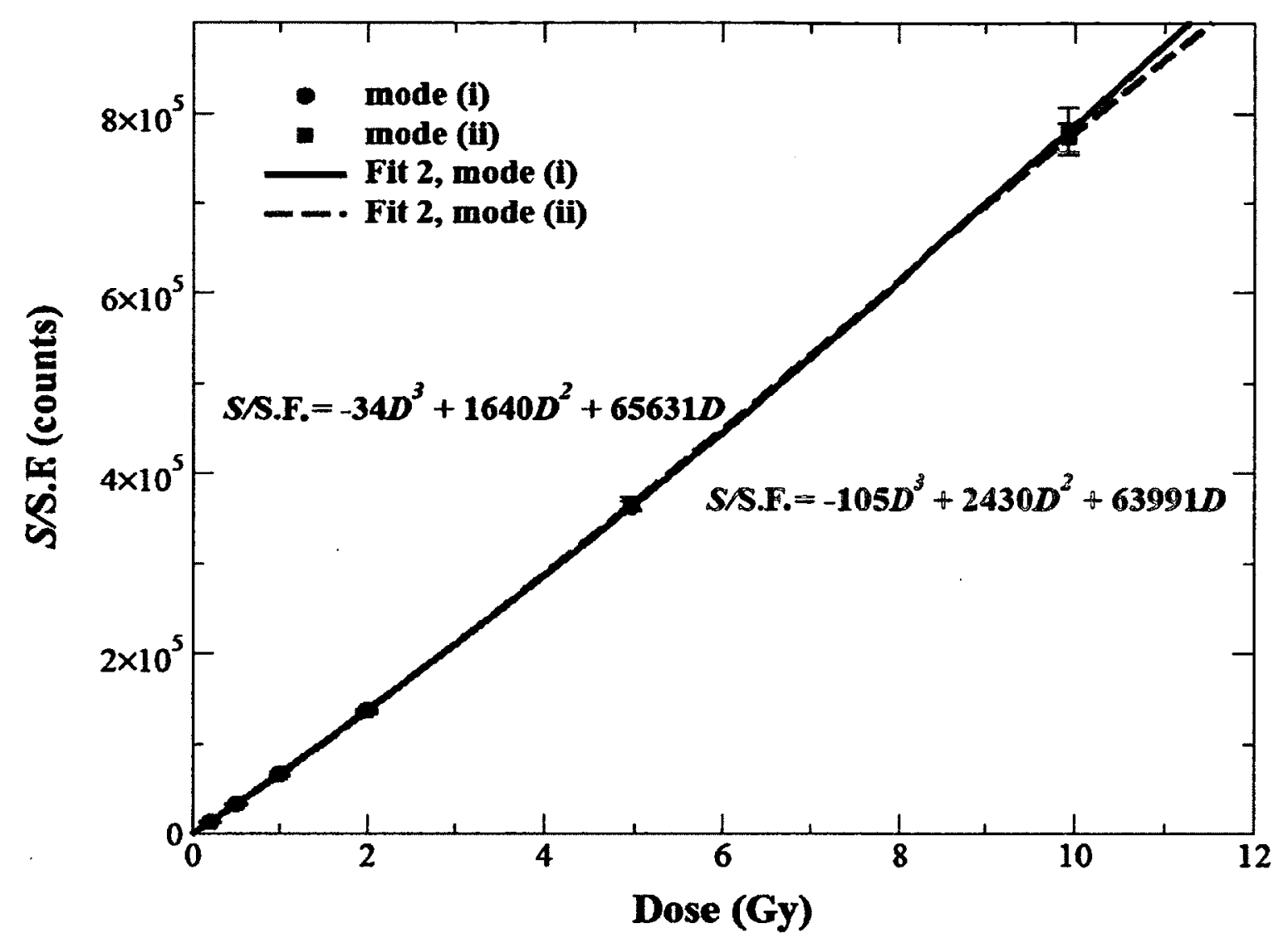

Figure 4-10: OSL signal $S$ divided by S.F., as a function of dose for nanoDot OSLDs exposed to doses $D$ from 0.5 to $10 \mathrm{~Gy}$. Each data point represents the average of three readouts of one nanoDot OSLD, except at 1 and $10 \mathrm{~Gy}$ where two OSLDs were used. Error bars are the standard deviation of the mean. The fit was determined using the model described by Eq. (3.6).

\subsubsection{Absorbed-dose determination}

Figure 4-11 shows the absorbed-dose determination using the individual calibration method, for the same nanoDot OSLDs used in Figure 4-7. Each data point represents the ratio of the OSLD measured dose DosL, determined by the solution to the calibration fitting models from Eq. (3.7) [Fit 1] and Eq. (3.8) [Fit 2], to the dose $D$ delivered per cycle to the nanoDot OSLDs, as measured by the ion chamber. Recall that the dose delivered to the OSLDs at cycle 3 for all dose fractions in Figure 4-7 was significantly lower, because the OSLDs may have been exposed to different irradiation conditions 
compared to the other cycles, hence, a lower OSLD measured dose was determined. Thus, throughout this work, data from cycle 3 were not included in our analysis.

Fit 1 and Fit 2 provided absorbed-dose estimates within $\pm 4 \%$ of the delivered dose, across all dose fractions regardless of bleaching mode for accumulated doses up to $50 \mathrm{~Gy}$. For accumulated doses above $50 \mathrm{~Gy}$, up to $6 \%$ absorbed-dose difference was observed for nanoDot OSLD bleached using mode (ii). However, Fit 1 and Fit 2 provided an underestimation and overestimation of the absorbed-dose $D_{\text {osL }}$ respectively, for nanoDot OSLDs accumulating low doses at $0.2 \mathrm{~Gy}$ dose fractions.

Table 4-8 shows the values of the chi-squared and modified chi-squared relationships for all dose fractions, comparing both absorbed-doses determined using Fit 1 and Fit 2, for the individual calibration method. It can be seen that Fit 2 gives the lowest values of $\chi^{2}$ and $\Gamma^{2}$ for both bleaching modes, except at 5 and $10 \mathrm{~Gy}$ where Fit 1 produces slightly lower values. However, at 0.2 Gy dose fractions, Fit 1 produces better dose determination using bleaching mode (ii), while Fit 2 produces better absorbed-dose estimations using bleaching mode (i). 

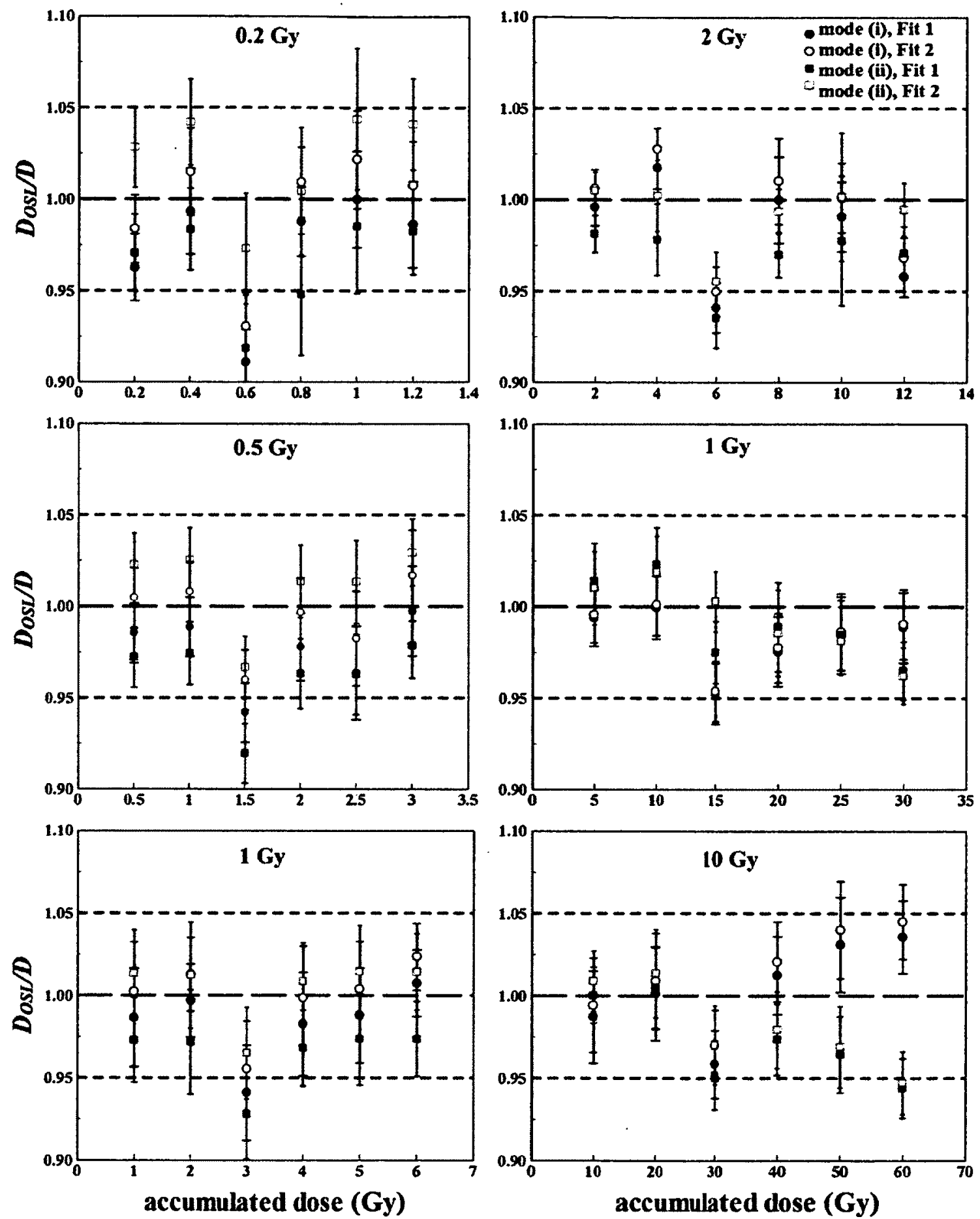

Figure 4-11: OSLD measured dose $D_{\text {ost }}$ divided by the delivered dose $D$, as a function of accumulated doses for individual calibration method. Bleaching of the nanoDot OSLDs was $10 \mathrm{~h}$ for mode (i) and $40 \mathrm{~h}$ for mode (ii) for all dose fractions. Each data point represents the ratio of the OSLD measured dose $D_{\text {osL }}$, determined by the calibration curves using fitting models from Eq.

(3.7) [Fit 1] and Eq. (3.8) [Fit 2], to the dose $D$ delivered per cycle to the nanoDot OSLDs, as measured by the ion chamber. Short dashed lines represent the $\pm 5 \%$ interval, from the expected unity value (long dash). 
Table 4-8: Values of the $\chi^{2}$ and $\Gamma^{2}$ statistics described by Eq. (3.9) and (3.10), comparing the two fitting models for dose estimations in Figure 4-11, using bleaching mode (i) and (ii).

\begin{tabular}{|c|c|c|c|c|}
\hline \multicolumn{5}{|c|}{ Bleaching mode (i) } \\
\hline & \multicolumn{2}{|c|}{$x^{2}$} & \multicolumn{2}{|c|}{$\Gamma^{2}$} \\
\hline Dose fractions & Fit 1 & Fit 2 & Fit 1 & Fit 2 \\
\hline $0.2 \mathrm{~Gy}$ & 5.05 & 1.93 & $6.506 \times 10^{-7}$ & $4.263 \times 10^{-7}$ \\
\hline $0.5 \mathrm{~Gy}$ & 4.81 & 1.18 & $1.128 \times 10^{-6}$ & $4.394 \times 10^{-7}$ \\
\hline $1 \mathrm{~Gy}$ & 0.85 & 1.06 & $6.147 \times 10^{-7}$ & $2.232 \times 10^{-7}$ \\
\hline $2 \mathrm{~Gy}$ & 17.19 & 14.93 & $2.901 \times 10^{-7}$ & $2.520 \times 10^{-7}$ \\
\hline $5 \mathrm{~Gy}$ & 2.70 & 3.68 & $3.846 \times 10^{-7}$ & $4.933 \times 10^{-7}$ \\
\hline $10 \mathrm{~Gy}$ & 4.26 & 4.27 & $1.619 \times 10^{-6}$ & $1.804 \times 10^{-6}$ \\
\hline \multicolumn{5}{|c|}{ Bleaching mode (ii) } \\
\hline & \multicolumn{2}{|c|}{$x^{2}$} & \multicolumn{2}{|c|}{$\Gamma^{2}$} \\
\hline Dose fractions & Fit 1 & Fit 2 & Fit 1 & Fit 2 \\
\hline $0.2 \mathrm{~Gy}$ & 5.69 & 6.82 & $3.963 \times 10^{-6}$ & $4.146 \times 10^{-6}$ \\
\hline $0.5 \mathrm{~Gy}$ & 13.29 & 4.78 & $1.704 \times 10^{-6}$ & $5.138 \times 10^{-7}$ \\
\hline $1 \mathrm{~Gy}$ & 6.09 & 0.52 & $2.725 \times 10^{-6}$ & $2.628 \times 10^{-7}$ \\
\hline $2 \mathrm{~Gy}$ & 14.95 & 1.15 & $1.170 \times 10^{-6}$ & $4.321 \times 10^{-8}$ \\
\hline $5 \mathrm{~Gy}$ & 7.54 & 10.18 & $7.737 \times 10^{-7}$ & $8.828 \times 10^{-7}$ \\
\hline $10 \mathrm{~Gy}$ & 13.62 & 13.62 & $2.046 \times 10^{-6}$ & $2.589 \times 10^{-6}$ \\
\hline
\end{tabular}

\subsubsection{Reference-dose calibration}

\subsubsection{Calibration curve}

Figure 4-12 shows the dose response for the same nanoDot OSLDs as in Figure 4-6, after exposure to doses from 0.2 to $10 \mathrm{~Gy}$ using the reference-dose calibration method. The dose response data were calibrated using the fit to the third-order model of Eq. (3.6) [Fit 2]. The fit to the second-order model of Eq. (3.5) [Fit 1] is also not shown here. 


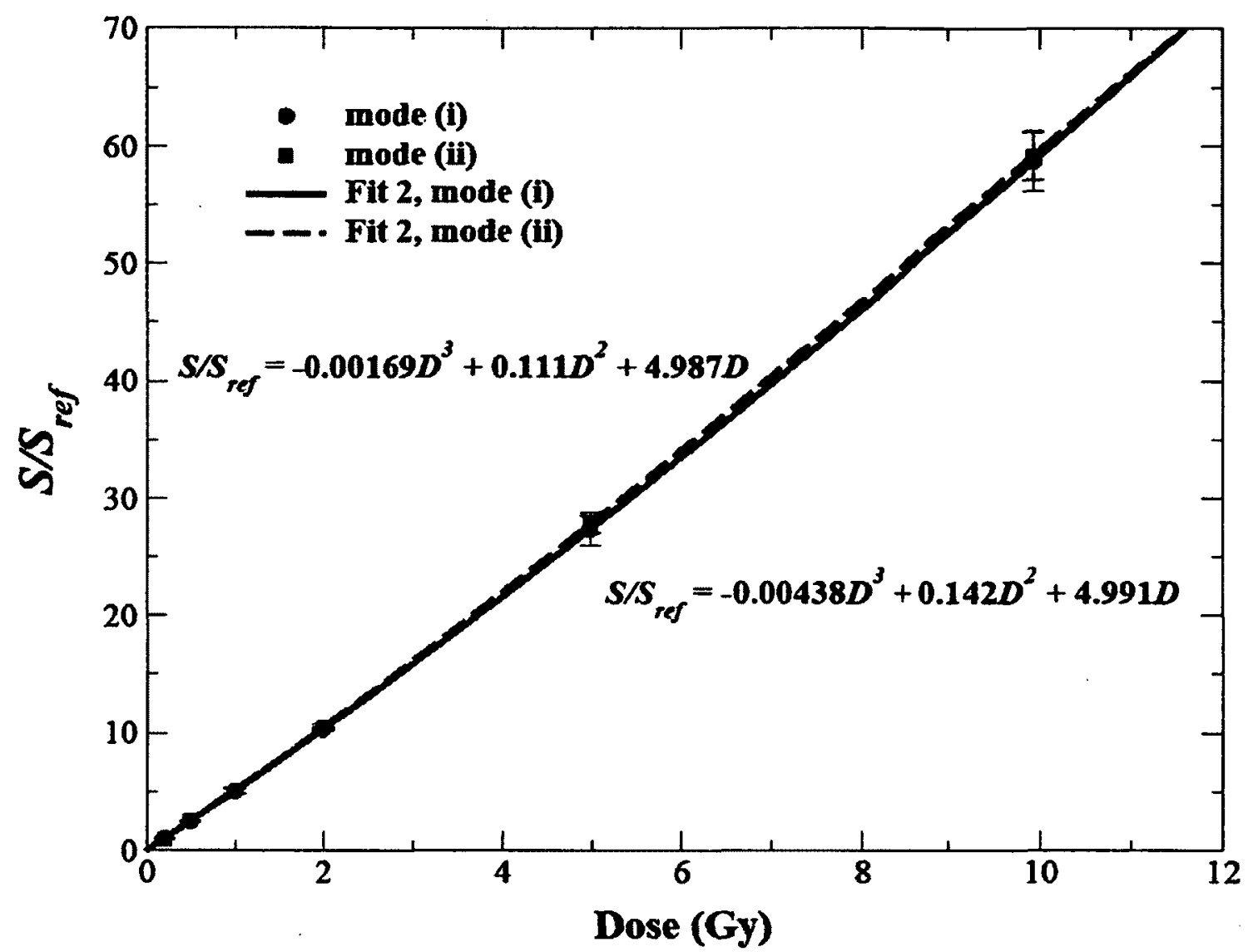

Figure 4-12: OSL signal $S$, divided by reference-dose signal $S_{\text {ref, }}$ as a function of dose for nanoDot OSLDs exposed to doses $D$ from 0.5 to $10 \mathrm{~Gy}$. $S_{\text {ref }}$ is the OSL reading after the reference dose of 20 cGy is delivered. Each data point represents the average of three readouts of one nanoDot OSLD, except at 1 and $10 \mathrm{~Gy}$ where two OSLDs were used. Error bars are the standard deviation of the mean. The fit was determined using the model described by Eq. (3.6).

\subsubsection{Absorbed-dose determination}

Figure 4-13 shows the absorbed-dose determination using the reference-dose calibration method, for the same nanoDot OSLDs used in Figure 4-7. Each data point represents the ratio of the OSLD measured dose $D_{O S L}$, determined by the solution to the calibration fitting models from Eq. (3.7) [Fit 1] and Eq. (3.8) [Fit 2], to the dose $D$ delivered per cycle to the nanoDot OSLDs, as measured by the ion chamber.

It can also be observed that Fit 2 provided the best absorbed-dose estimates $( \pm$ 3.5\%) across all dose fractions regardless of bleaching mode. However, both Fit 1 and Fit 
2 do not provide good estimates of OSLD measured dose $D_{\text {OSL }}$ when accumulating doses more than $20 \mathrm{~Gy}$, where up to $18 \%$ change was observed for nanoDot OSLDs bleached with filter (mode ii) after $40 \mathrm{~Gy}$ accumulated dose.

Table 4-9 shows the values of the chi-squared and modified chi-squared relationships for all dose fractions, comparing both absorbed-doses determined using Fit 1 and Fit 2, for the reference-dose calibration method. It can be seen that Fit 2 gives the lowest values of $\Gamma^{2}$ for both bleaching modes, except 10 Gy where Fit 1 produces lower values of $\Gamma^{2}$ and slightly higher values of $\chi^{2}$ for both bleaching modes. Hence, Fit 2 provided the best absorbed-dose estimations.

Table 4-9: Values of the $\chi^{2}$ and $\Gamma^{2}$ statistics described by Eq. (3.9) and (3.10), comparing the two fitting models for dose estimations in Figure 4-13, using bleaching mode (i) and (ii).

\begin{tabular}{l}
\multicolumn{5}{|c|}{ Bleaching mode (i) } \\
\hline
\end{tabular}



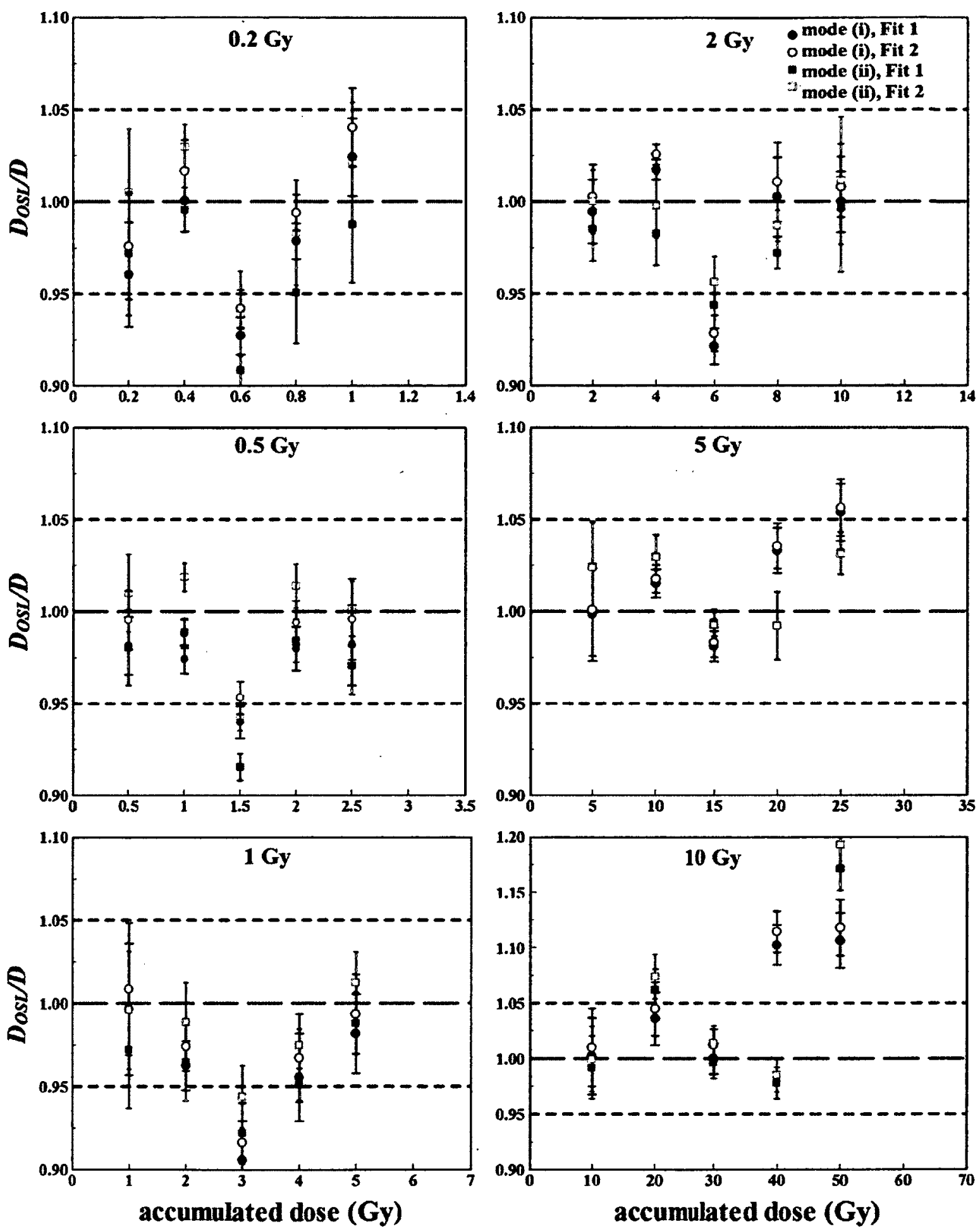

Figure 4-13: OSLD measured dose $D_{\text {osL }}$ divided by the delivered dose $D$, as a function of accumulated doses for the reference-dose calibration method. Each data point represents the ratio of the OSLD measured dose $D_{\text {os }}$, determined by the calibration curves using fitting models from Eq.

(3.7) [Fit 1] and Eq. (3.8) [Fit 2], to the dose $D$ delivered per cycle to the nanoDot OSLDs, as measured by the ion chamber. Short dashed lines represent the $\pm 5 \%$ interval, from the expected unity value (long dash). Bleaching of the nanoDot OSLDs was $10 \mathrm{~h}$ for mode (i) and $40 \mathrm{~h}$ for mode (ii) for all dose fractions. 


\subsubsection{Pre-dose calibration}

\subsubsection{Regeneration of OSL signals}

Using the saturated-exponential function as described in Section 3.6.1, we obtained risetime values of $560 \mathrm{~h}$ and $564 \mathrm{~h}$, for bleaching modes (i) and (ii), respectively as shown in Figure 4-14. At the saturation level $A$, the regenerated signal corresponded to doses of approximately $1.18 \mathrm{~Gy}$ and $0.38 \mathrm{~Gy}$ for bleaching modes (i) and (ii), respectively. After $48 \mathrm{~h}$, the equivalent doses were about $0.23 \mathrm{~Gy}$ and $0.07 \mathrm{~Gy}$.

The same nanoDot OSLDs used to obtain the data in Figure 4-14 were rebleached for $10 \mathrm{~h}$ in mode (i) and $24 \mathrm{~h}$ in mode (ii). After bleaching, the OSL signals corresponded to residual signals of approximately $0.2 \mathrm{cGy}$ and $0.33 \mathrm{cGy}$ respectively. Thereafter, the OSLDs were again stored and the regeneration of the signals reinvestigated as a function of storage time for both bleaching mode (i) and (ii). Figure 4-15 shows the regeneration of the signals for the same nanoDot OSLDs after rebleaching them. For the second regeneration, we obtained rise-time values of $956 \mathrm{~h}$ and $424 \mathrm{~h}$ after storage in dark for $225 \mathrm{~h}$ and $215 \mathrm{~h}$, for bleaching modes (i) and (ii), respectively. 


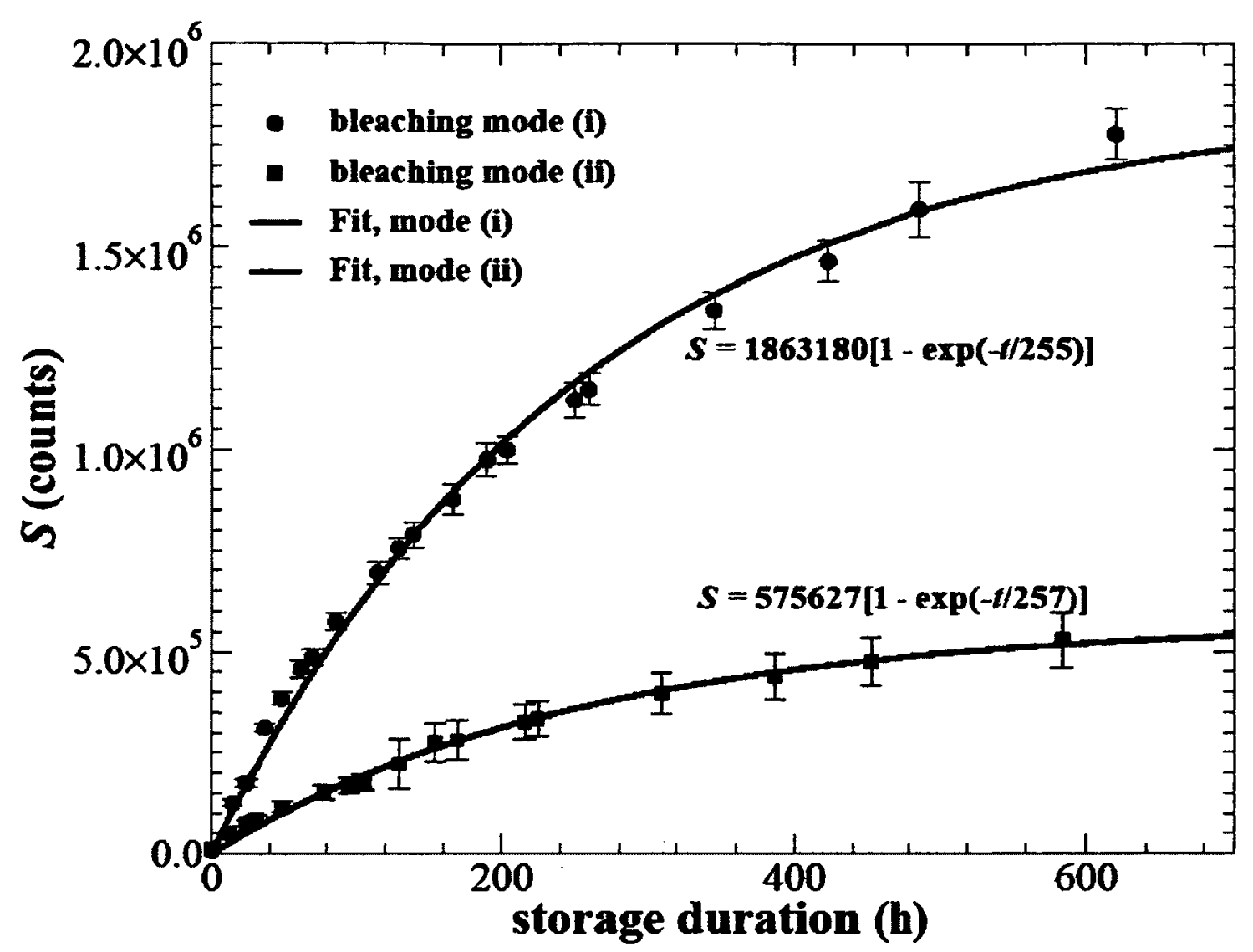

Figure 4-14: Regeneration of the OSL signal, $S$ as a function of storage duration, $t$. The OSLDs were pre-exposed to $1 \mathrm{kGy}$, and then bleached with mode (i) for $12 \mathrm{~h}$ and mode (ii) for $45 \mathrm{~h}$ to very low residual OSL signal levels equivalent to $\sim 0.5 \mathrm{cGy}$ and $\sim 0.8 \mathrm{cGy}$, respectively. Each data point represents the mean of 9 nanoDot OSLDs and error bars are the standard deviation of the mean. 


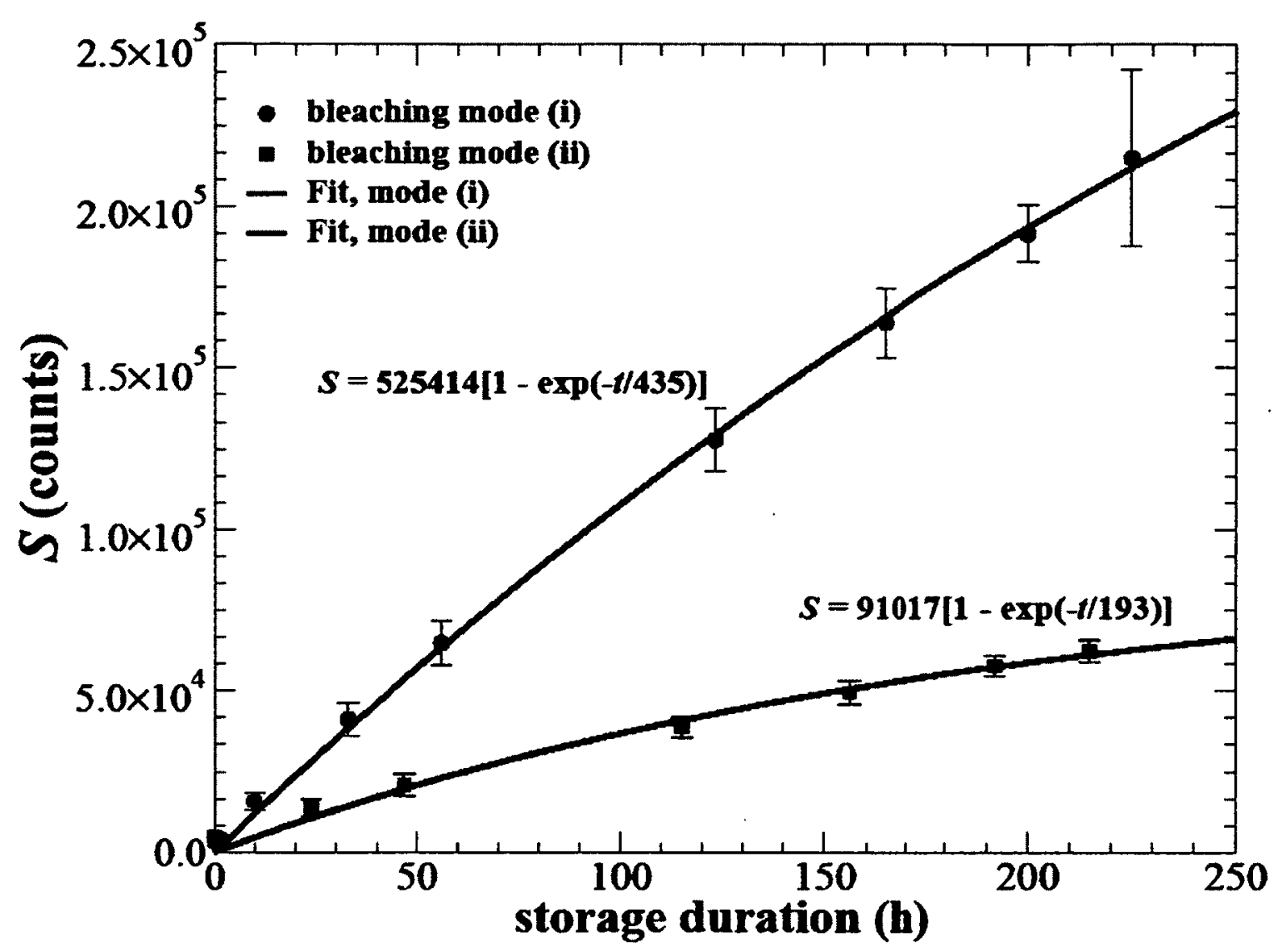

Figure 4-15: Regeneration of the OSL signal $S$ as a function of storage time, $t$, for nanoDot OSLDs used in Figure 4-14, after re-bleaching to low residual OSL signals equivalent to $\sim 0.2 \mathrm{cGy}$ and $\sim 0.33$

cGy, using bleaching mode (i) and (ii) respectively. The bleaching of the OSLDs was after regeneration of the OSL signal to the saturation level $A$ described in the text. Each data point represents the mean of 9 nanoDot OSLDs and error bars are the standard deviation of the mean.

\subsubsection{Calibration curve}

The calibration curves for the nanoDot OSLDs pre-exposed to $1 \mathrm{kGy}$ follow the definitions from calibration methods a), b) and c), (see Figure 4-16 to Figure 4-18). The data were fit to the third-order polynomial model as shown in Eq. (3.6). These nanoDot OSLDs were irradiated to the doses $D$, immediately after bleaching to account for the regeneration of the OSL signals as shown above. 


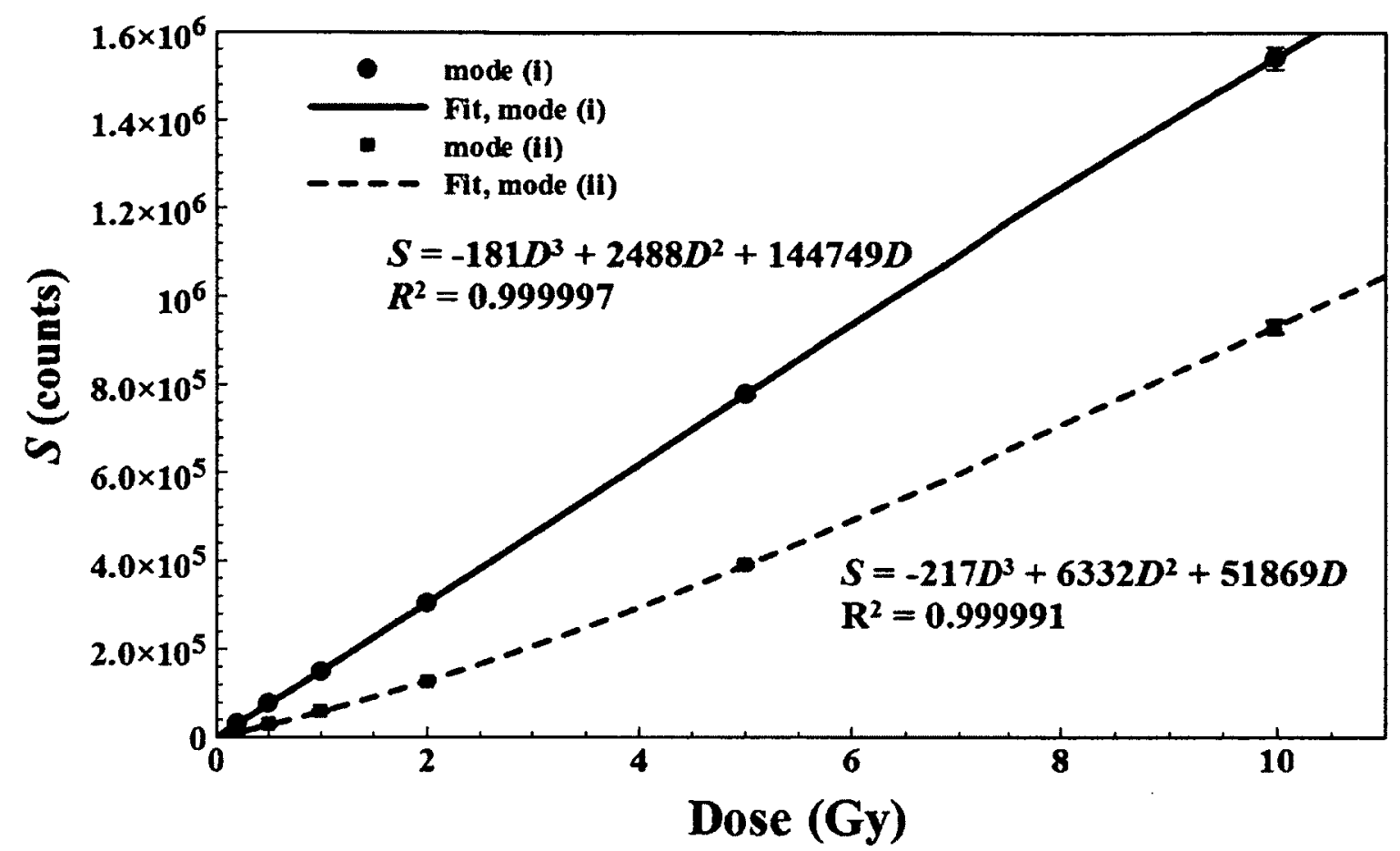

Figure 4-16: OSL signal $S$ as a function of dose for OSLDs exposed to pre-dose of $1 \mathrm{kGy}$, bleached to low residual signal, and irradiated with doses $D$ from 0.5 to $10 \mathrm{~Gy}$. Each data point represents the average of three readouts of one nanoDot OSLD, except at 1 and $10 \mathrm{~Gy}$ where two OSLDs were used. Error bars are the standard deviation of the mean. The fit was determined using the model described by Eq. (3.6).

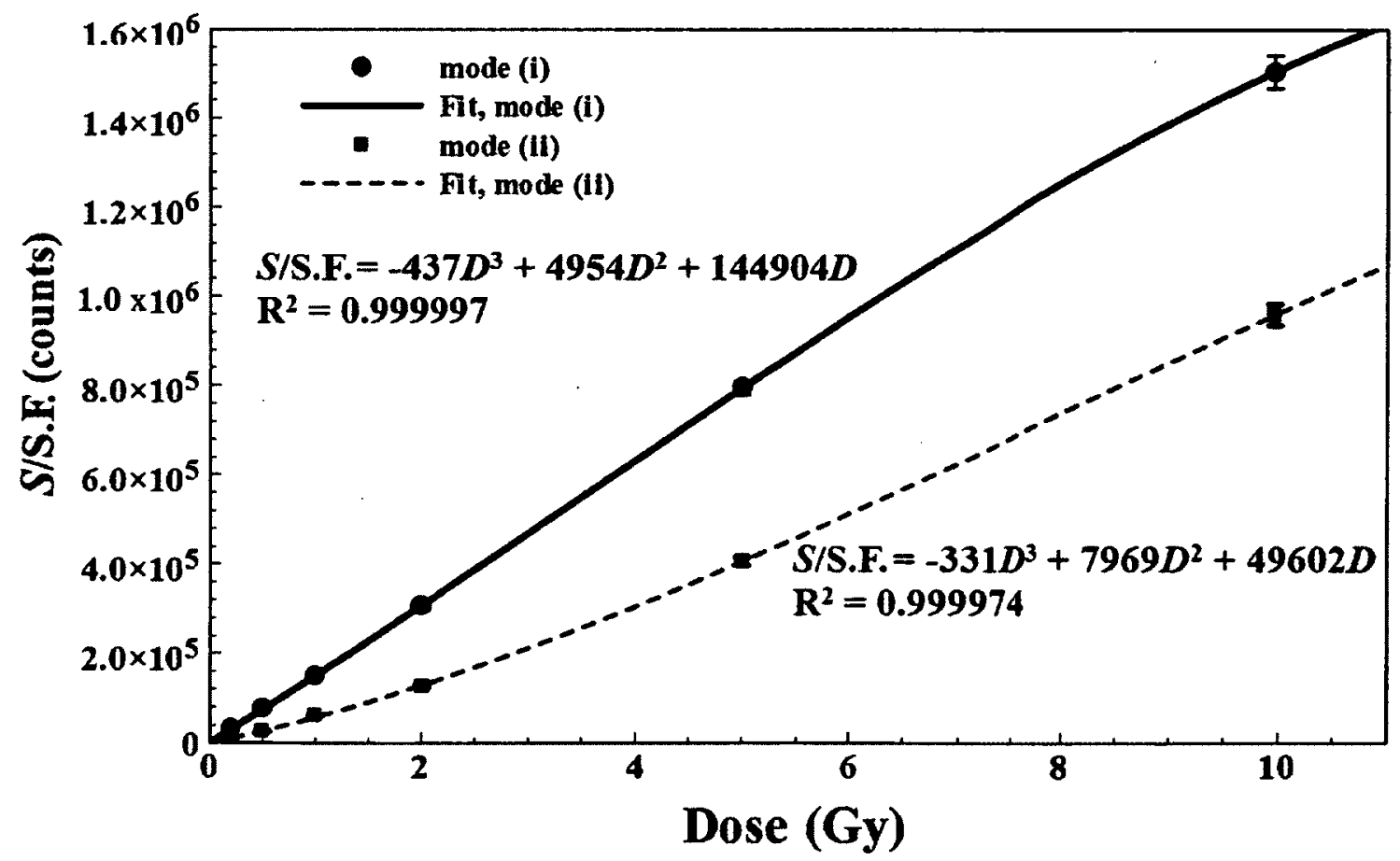

Figure 4-17: OSL signal $S$, divided by S.F., as a function of dose for nanoDot OSLDs exposed to predose of $1 \mathrm{kGy}$, bleached and irradiated with doses $D$ from 0.5 to $10 \mathrm{~Gy}$. Data points are as described in Figure 4-16. 


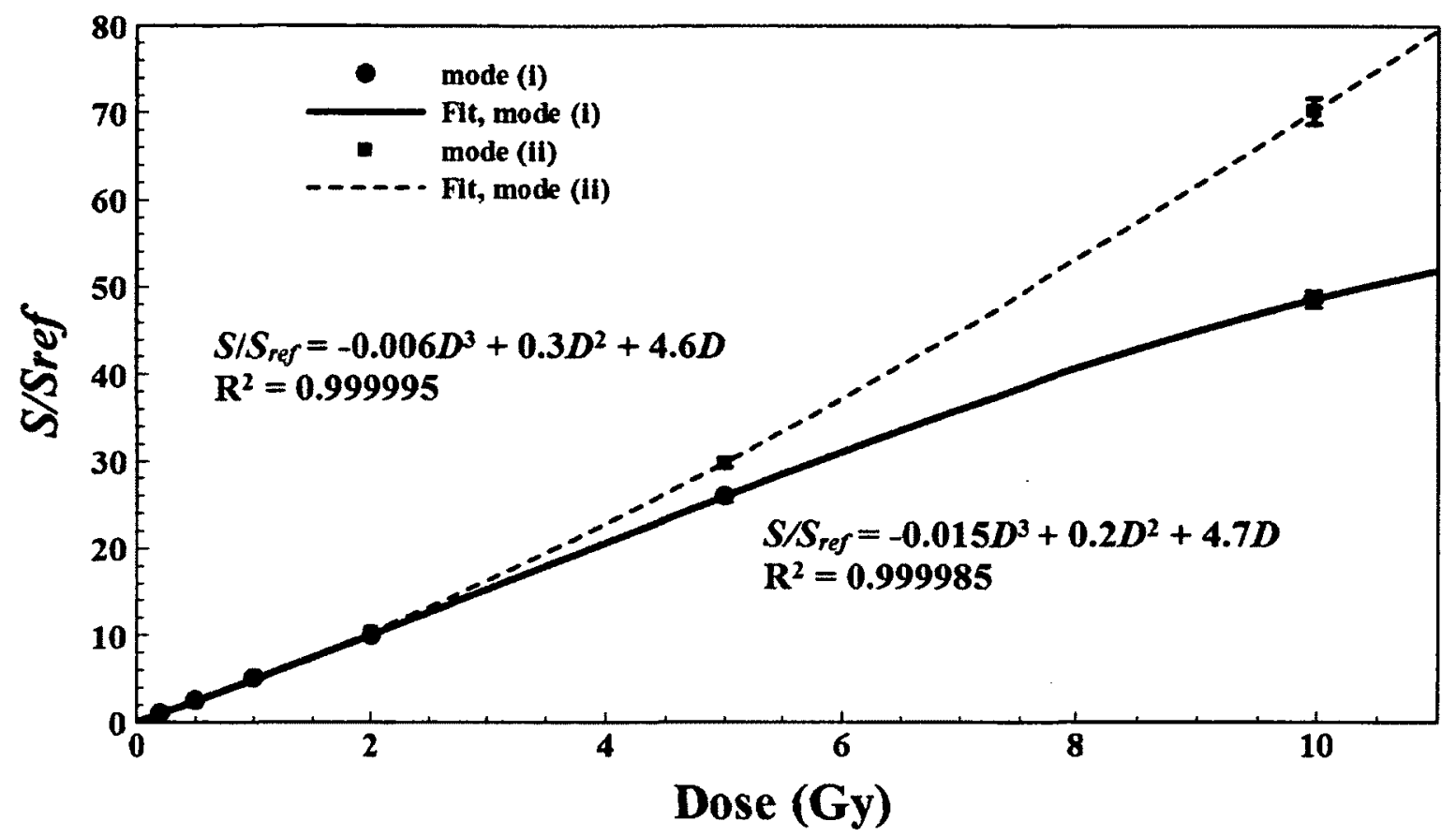

Figure 4-18: OSL signal $S$, divided by reference OSL signal $S_{\text {ref, }}$ as a function of dose for nanoDot OSLDs exposed to a pre-dose of $1 \mathrm{kGy}$, bleached to low residual signals and exposed to doses $D$ from 0.5 to 10 Gy. Data points are as described in Figure 4-16.

\subsubsection{Absorbed-dose determination}

Figure 4-19 - Figure 4-21 shows the absorbed-dose determination for nanoDot OSLDs for the pre-dose calibration method, using the calibration method a), b) and c) respectively.

It can be observed that all the calibration methods produce over $\pm 5 \%$ sensitivity change for bleaching mode (ii) across all dose fractions and calibration fits, except at 10 Gy fractions where less than $\pm 2 \%$ absorbed-dose differences were observed. However, using bleaching mode (i) produced less than $\pm 3 \%$ absorbed-dose differences for all dose fractions using Eq. (3.8) - Fit 2 - for the absorbed-dose calculation.

The most significant reason for the high absorbed-dose determination is that for this calibration method, nanoDot OSLDs were read out in different stimulation modes of 
the reader, depending on the bleaching mode employed. This is due to the different regeneration in the OSL signal; hence the reader switches from the weak- to the strongstimulation readout mode for the reference-dose calibration method. Recalling from Section 4.4, the use of scaling factors is required to convert the OSL readings to dose, when nanoDot OSLDs are read out in both the strong- and weak stimulation modes of the reader. NanoDot OSLDs exhibited lower sensitivity at the higher accumulated doses (10 Gy fractions), such that after exposure to the 20 cGy reference doses, they produce low OSL signals requiring switch from the weak- to the strong-stimulation mode of the reader between the two readings of $S$ and $S_{\text {ref. }}$. Hence, the requirement of scaling factors is a drawback in the methodology for this calibration protocol. 

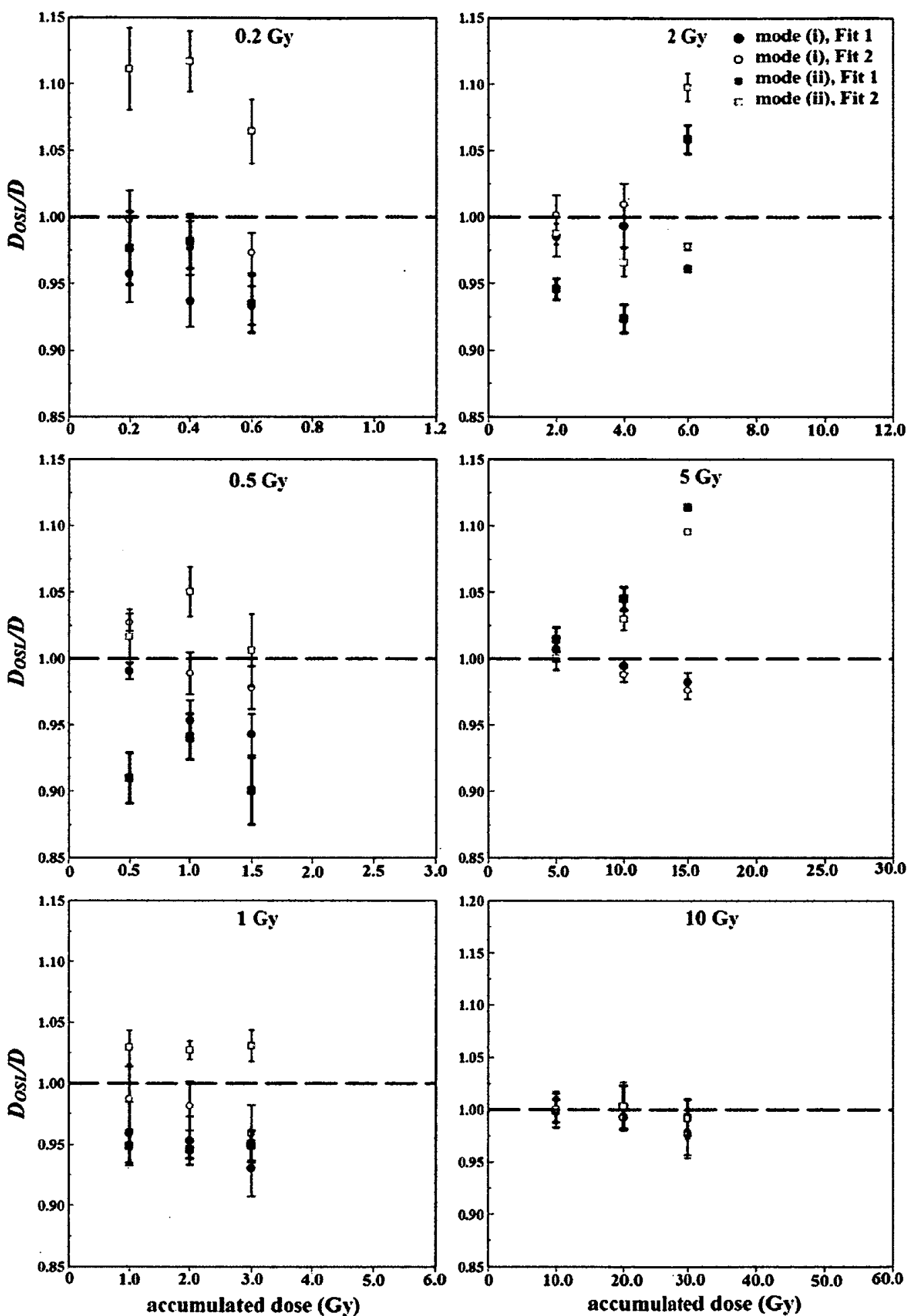

Figure 4-19: OSLD measured dose $D_{\text {osL }}$ divided by the delivered dose $D$, as a function of cycle for pre-dose calibration method using the individual calibration definition. Each data point represents the ratio of the OSLD measured dose $D_{\text {osL }}$, determined by the calibration curves using fitting models from Eq. (3.7) [Fit 1] and Eq. (3.8) [Fit 2], to the dose $D$ delivered per cycle to the nanoDot OSLDs, as measured by the ion chamber. Dashed lines represent the expected unity value. Bleaching of the nanoDot OSLDs was $10 \mathrm{~h}$ for mode (i) and $40 \mathrm{~h}$ for mode (ii) for all dose fractions. 

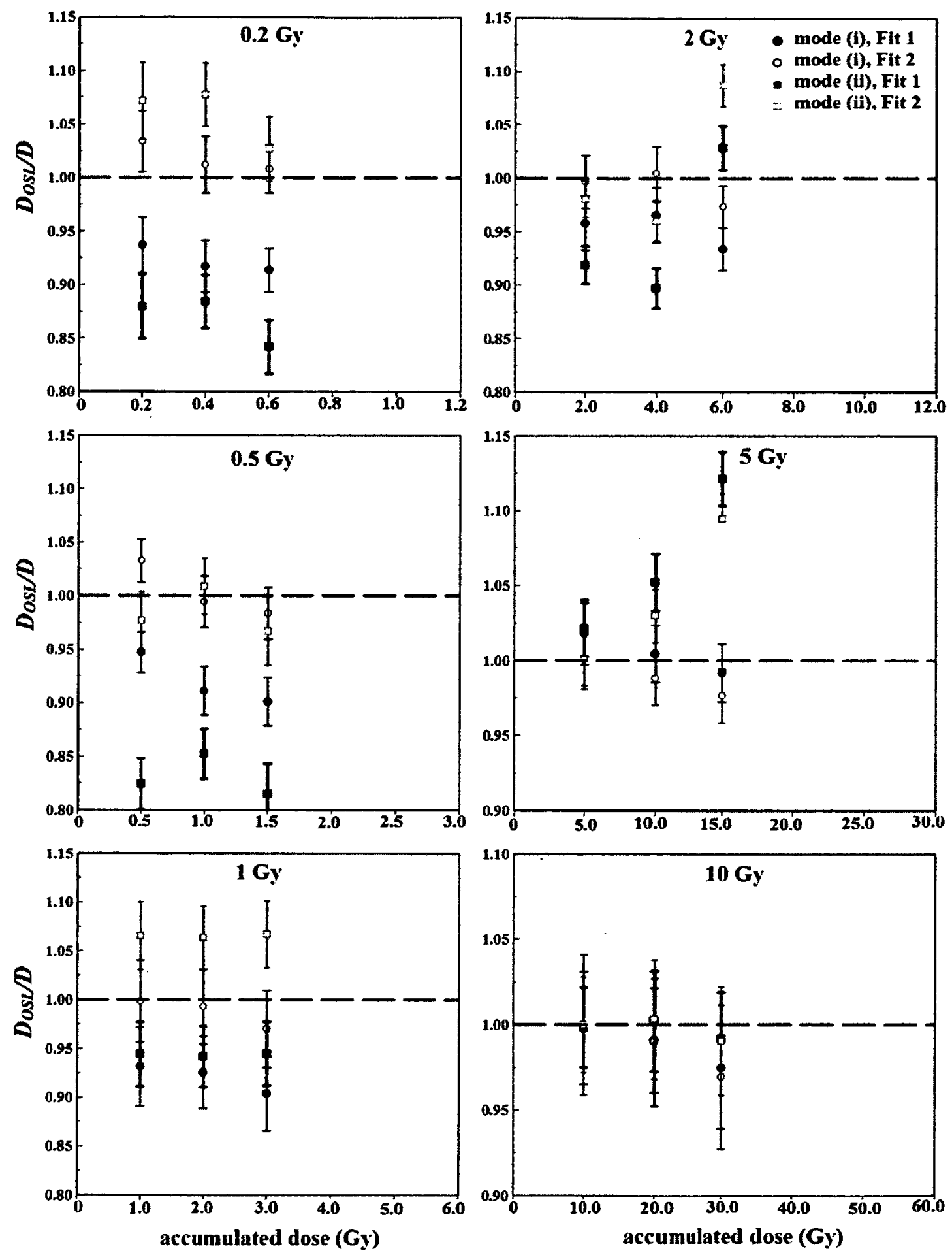

Figure 4-20: OSLD measured dose $D_{\text {osL }}$ divided by the delivered dose $D$, as a function of cycles for

the pre-dose calibration method using the individual calibration definition. Each data point represents the ratio of the OSLD measured dose $D_{\text {osL }}$, determined by the calibration curves using fitting models from Eq. (3.7) [Fit 1] and Eq. (3.8) [Fit 2], to the dose $D$ delivered per cycle to the nanoDot OSLDs, as measured by the ion chamber. Dashed lines represent the expected unity value. Bleaching of the nanoDot OSLDs was $10 \mathrm{~h}$ for mode (i) and $40 \mathrm{~h}$ for mode (ii) for all dose fractions. 

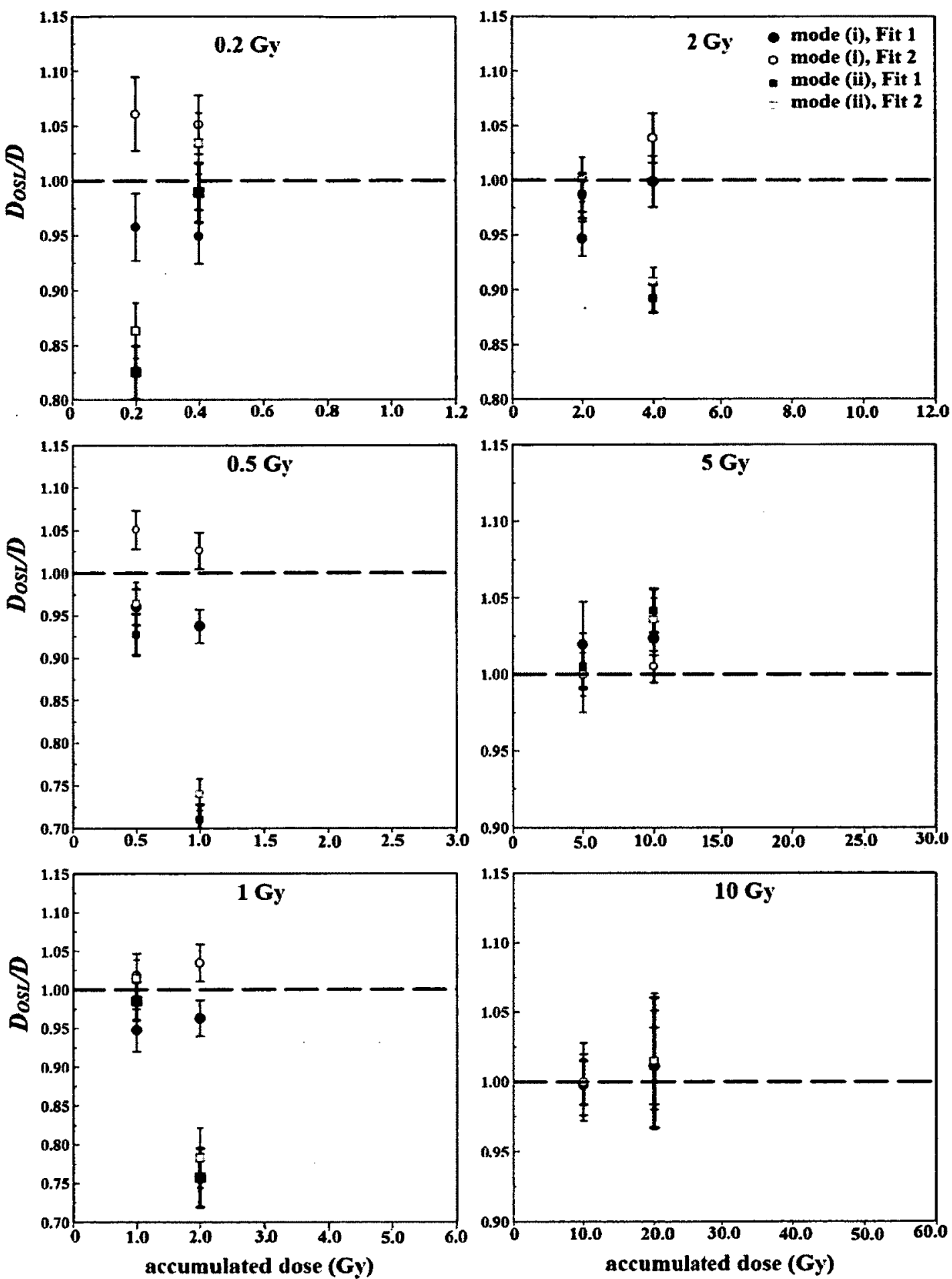

Figure 4-21: OSLD measured dose $D_{\text {osL }}$ divided by the delivered dose $D$, as a function of cycles for the pre-dose calibration method using the reference-dose calibration definition. Each data point represents the ratio of the OSLD measured dose $D_{\text {osL, }}$, determined by the calibration curves using fitting models from Eq. (3.7) [Fit 1] and Eq. (3.8) [Fit 2], to the dose $D$ delivered per cycle to the nanoDot OSLDs, as measured by the ion chamber. Dashed lines represent the expected unity value. Bleaching of the nanoDot OSLDs was $10 \mathrm{~h}$ for mode (i) and $40 \mathrm{~h}$ for mode (ii) for all dose fractions. 


\subsubsection{Comparison of absorbed-dose determination for all calibration protocols}

The comparison of the absorbed-dose determination for the three calibration methods batch, individual and reference-dose - using Eq. (3.8) and Eq. (3.10) are shown in Figure 4-22 and Figure 4-23, for bleaching modes (i) and (ii), respectively. Recalling from Page 60 , we mentioned that the two fitting models produced different dose estimations at 0.2 Gy dose fractions, which are not relevant to radiotherapy applications. Henceforth, we would adopt Fit 2 - Eq. (3.8) and Eq. (3.10) - when comparing the OSL unknown doses $D_{\text {OSL }}$ to the actual delivered doses $D$.

The absorbed-dose determined using the batch calibration method [Figure 4-7] and the individual calibration method [Figure 4-11], produced similar results within $\pm 3.5 \%$ for all dose fractions and bleaching modes, except at 0.2 Gy and $10 \mathrm{~Gy}$ dose fractions where up to $5.5 \%$ absorbed-dose differences were observed for the batch calibration method. However, the reference-dose calibration method [Figure 4-13] produced the best dose estimates $( \pm 3.8 \%)$ at 0.2 Gy dose fractions, but significantly large absorbed-dose differences of approximately $17 \%$ for bleaching with filter (mode ii) and approximately $10 \%$ for nanoDot OSLDs bleached without filter (mode i), at $50 \mathrm{~Gy}$ accumulated dose (10 Gy dose fractions).

The reason for the high absorbed-dose differences have been stated in Section

4.5.4.3. It is due to the requirement and use of scaling factors to convert OSL readings for nanoDot OSLDs read in the two stimulation modes of the reader, leading to inaccuracies in the determined dose.

Fit 2 - Eq. (3.8) and Eq. (3.10) - provided the best absorbed-dose differences for all cycles, regardless of the bleaching mode employed. However, it should be emphasized 
that at 5 and 10 Gy dose fractions, both fits agreed with each other within experimental uncertainties. The individual calibration method produced the best absorbed-dose estimations $( \pm 3.5 \%)$ when compared to the batch and reference-dose calibration methods, producing up to $5.5 \%$ and $18 \%$ absorbed-dose differences, at the highest accumulated dose of $60 \mathrm{~Gy}$ (10 Gy fractions).

It should also be emphasized that the batch and individual calibration methods produced dose estimates within $2.6 \%$ for all the dose fractions and bleaching modes investigated. The reference dose calibration method was inferior, producing up to $8 \%$ dose estimates at $10 \mathrm{~Gy}$ dose fractions. 

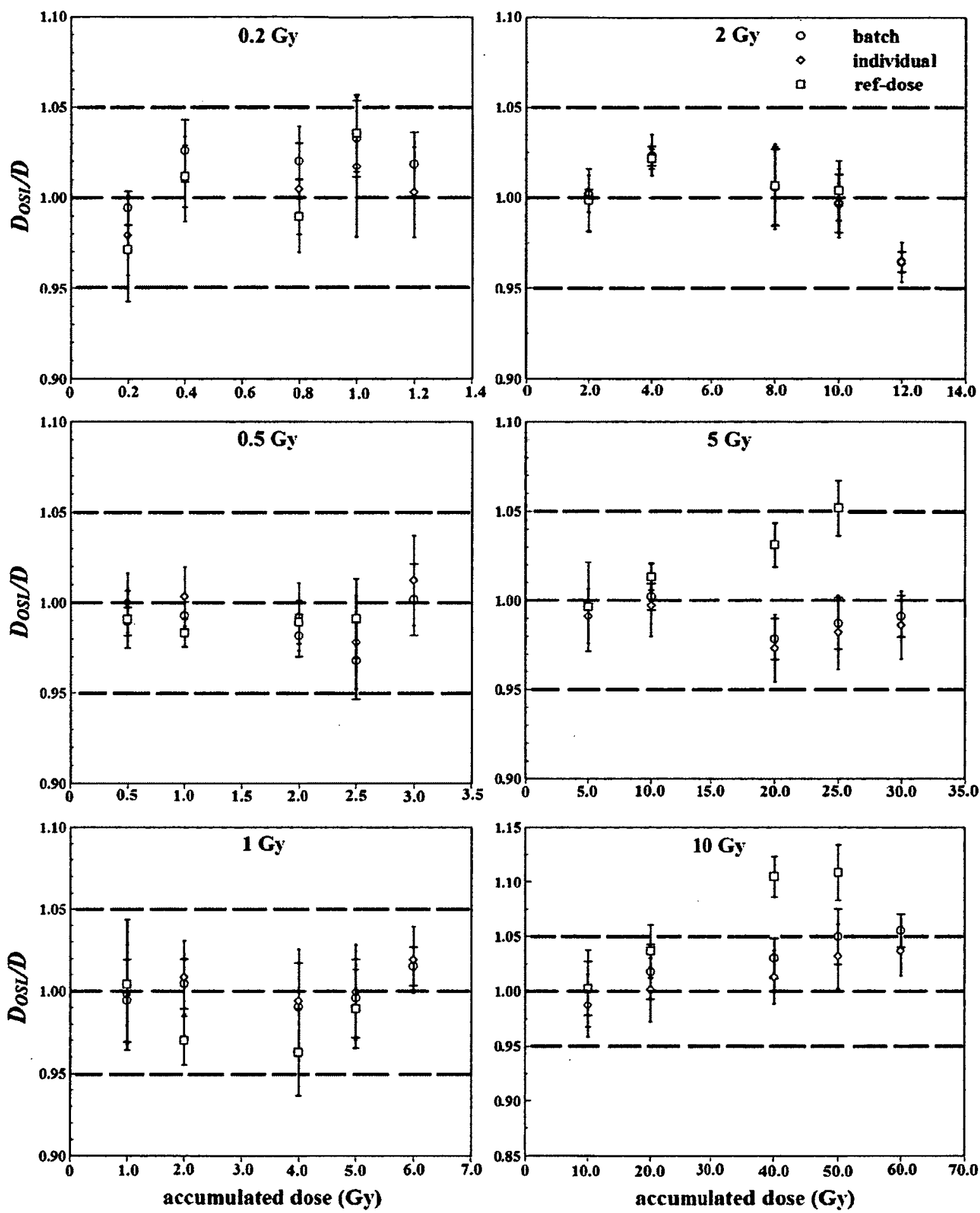

Figure 4-22: OSLD measured dose $D_{\text {ost }}$ divided by the delivered dose $D$, as a function of accumulated doses for all calibration methods. Each data point represents the ratio of the OSLD measured dose $D_{\text {osL, }}$, determined by the calibration curves using fitting model from Eq. (3.8) [Fit 2], to the dose $D$ delivered per cycle to the nanoDot OSLDs, as measured by the ion chamber. Dashed lines represent the $\pm 5 \%$ interval, from the expected unity value. Bleaching of the nanoDot OSLDs was $10 \mathrm{~h}$ using mode (i) for all dose

fractions. 

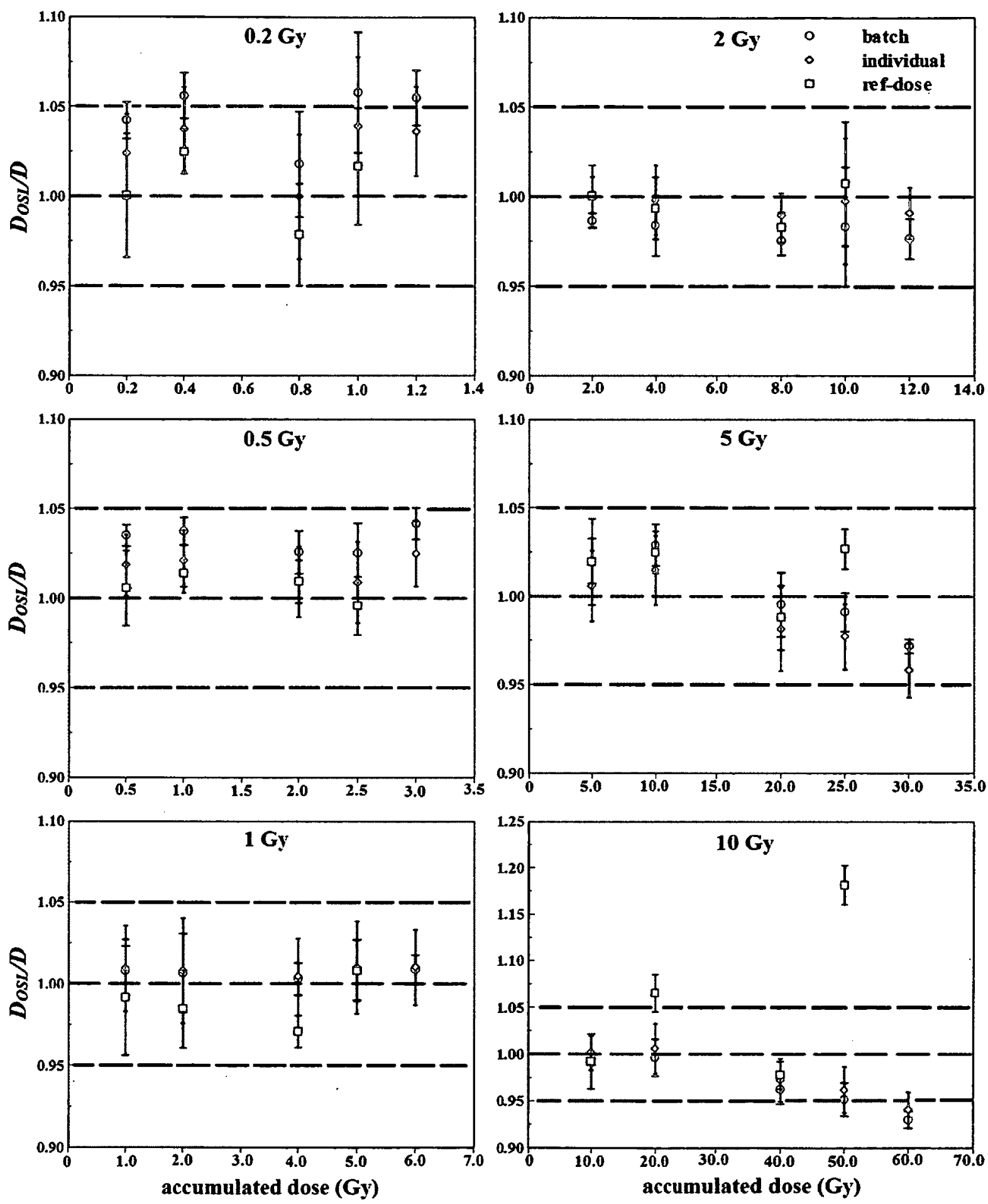

Figure 4-23: OSLD measured dose $D_{\text {OSL }}$ divided by the delivered dose $D$, as a function of accumulated doses for all calibration methods. Each data point represents the ratio of the OSLD measured dose $D_{\text {osL }}$, determined by the calibration curves using fitting model from Eq. (3.8) [Fit 2], to the dose $D$ delivered per cycle to the nanoDot OSLDs, as measured by the ion chamber. Dashed lines represent the $\pm 5 \%$ interval, from the expected unity value. Bleaching of the nanoDot OSLDs was $40 \mathrm{~h}$ using mode (ii) for all dose fractions. 


\subsection{Dose response and supralinearity per cycle for pre-dosed nanoDot OSLDs}

Figure 4-24 shows the dose response plots per irradiation-readout-bleaching cycle for the 16 nanoDot OSLDs pre-exposed to $1 \mathrm{kGy}$, using both bleaching modes (i) and (ii). The data were fit to Eq. (3.6) as in Section 3.7. Each data point represents the average of three readouts of a single nanoDot OSLD, with error bars representing the standard deviation of the mean including statistical fluctuations.

Figure 4-25 shows a plot of the supralinearity index per cycle for the pre-dosed nanoDot OSLDs using Eq. (3.3). The plot shows the supralinearity increases by $58 \%$ until 10 Gy for nanoDot OSLDs bleached with filter - mode (ii) - for all cycles investigated; while the increase was only $\sim 3-4 \%$ for nanoDot OSLDs bleached without filter - mode (i) - for all cycles reaching saturation after $5 \mathrm{~Gy}$. 

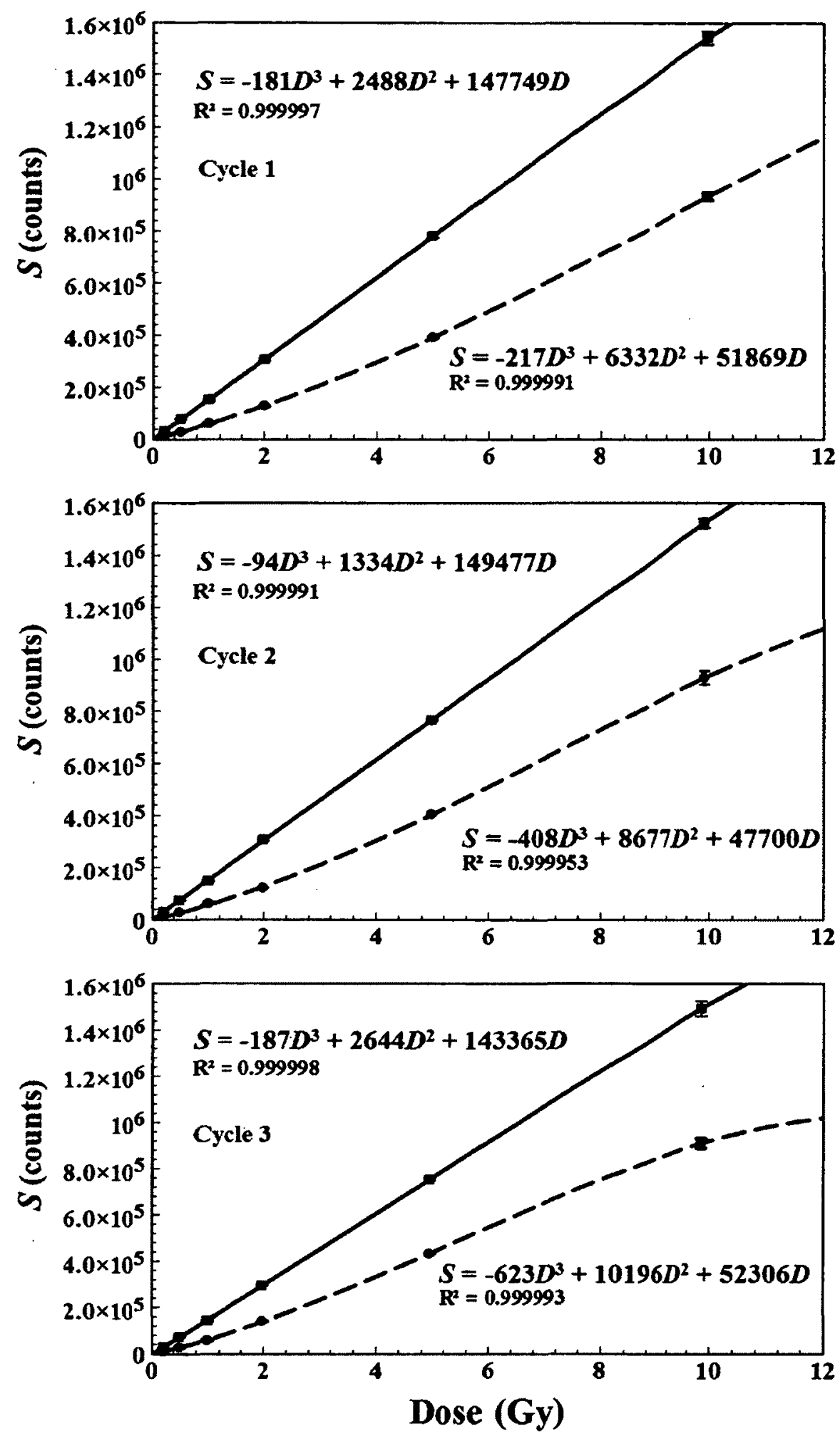

Figure 4-24: OSL signal $S$ as a function of dose per irradiation-readout-bleaching-readout cycle, for OSLDs pre-exposed to $1 \mathrm{kGy}$, bleached to low residual signals, and irradiated with doses $D$ from 0.5 to $10 \mathrm{~Gy}$. The black solid line represents the fit to the OSL signal after bleaching with mode (i), while the red dashed line is the fit to the signal after bleaching with mode (ii). The fits were determined using the model described by Eq. (3.6). 

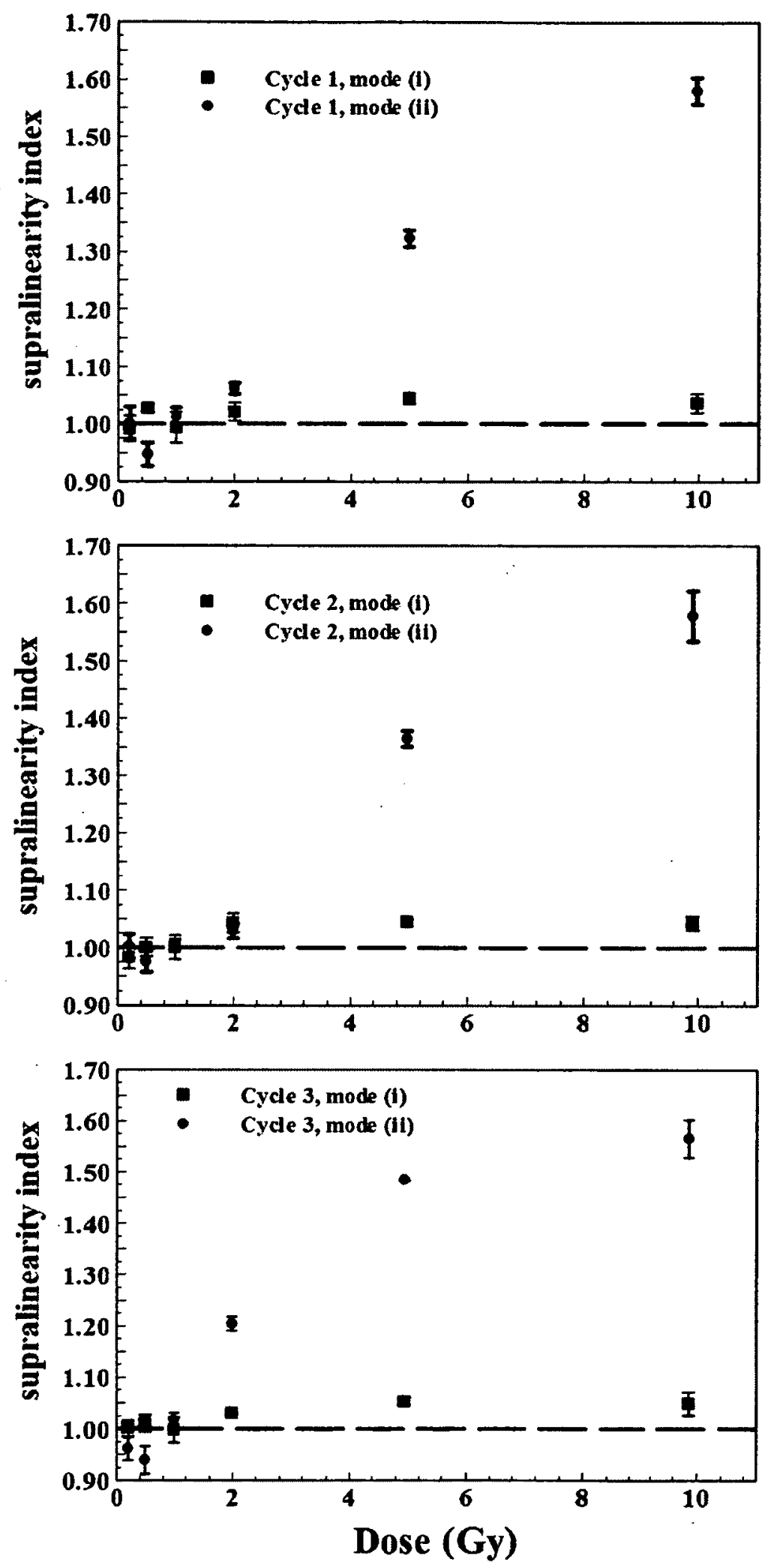

Figure 4-25: Supralinearity plots per irradiation-readout-bleaching cycle for nanoDot OSLDs exposed to $1 \mathrm{kGy}$ pre-dose, using both bleaching modes (i) and (ii). Each data point shows the supralinearity index described by Eq. (3.3). 


\section{Discussion}

\subsection{Dose response}

We observe a linear response in dose until 2 Gy (see Figure 4-1). At doses of 2 Gy and above, a supra-linear response is seen until the maximum dose used in this study of 10 Gy. Note that we assumed a linear dose response behavior until $1 \mathrm{~Gy}$. However, less than $0.6 \%$ differences in the supralinearity index values were observed if we only used the dose at $0.5 \mathrm{~Gy}$ to define the linear behavior of the dose response.'

Since the dose response is not linear, we used second- and third-order polynomial models to fit the data (Figure 4-1). The comparison between the two fitting models using Fisher's F-test showed that the second-order polynomial function (Fit 1) produced the best fit to the dose response, giving an F-statistic of 3.88, with a p-value of 0.147 .

Viamonte et al., 2008 observed a linear response in $\mathrm{Al}_{2} \mathrm{O}_{3}: \mathrm{C}$ Dot OSLDs exposed from 0.5 to $4 \mathrm{~Gy}^{22}$ while other studies have also reported a supra-linear behavior in $\mathrm{Al}_{2} \mathrm{O}_{3}: \mathrm{C}$ OSLDs. ${ }^{24,33,34,36,70} \mathrm{~A}$ non-linear regression model can be used to calibrate the OSLD dose response, hence creating a calibration curve within the entire dose range. Jursinic, 2010 fit an exponential model to nanoDot OSLDs exposed to doses between 0.2 Gy and $10 \mathrm{~Gy}$, reporting an accuracy within $0.4 \%$ to the delivered doses. ${ }^{70}$ Mrčela et al., 2011 used a quadratic fit to describe the data for $\mathrm{Al}_{2} \mathrm{O}_{3}: \mathrm{C}$ Dot OSLDs exposed in the same dose range. ${ }^{33}$

\subsection{Loss of OSL signal as a function of sequential readouts}

The faster rate of loss of the OSL signal for the strong-stimulation readout mode was expected because the MicroStar reader utilizes a 'strong' LED intensity to stimulate 
OSLDs exposed to doses less than approximately 0.1 Gy and a 'weak' LED intensity to stimulate OSLDs exposed to doses larger than approximately $0.1 \mathrm{~Gy}$. Hence, nanoDot OSLDs can be read multiple times using the weak-stimulation mode of the MicroStar reader without significant loss of the OSL signal. The rate of loss of the OSL signal can also be seen to depend on dose as the nanoDot OSLDs exposed to higher doses showed a higher rate of loss of the OSL signal. ${ }^{1}$ The nanoDot OSLDs exposed to $10 \mathrm{~Gy}$ showed the highest rates of loss of the OSL signal at $(0.031 \pm 0.001) \%$ per readout. OSLDs exposed to 0.2 and 0.5 Gy showed rates of $(0.019 \pm 0.002) \%$ per readout and those exposed to 1 Gy showed rates of $(0.024 \pm 0.001) \%$ per readout [see Table 4-2].

Previous studies ${ }^{24,33}$ have reported rates of loss of the OSL signal in the interval from $0.04 \%$ to $0.05 \%$. The manufacturer reports rates of loss of the OSL signal of approximately $0.25 \%$ and $0.04 \%$ for the strong- and weak-stimulation readout modes, respectively. We should also mention that the rate of loss of the OSL signal is highly dependent on the reader and the stimulation time used. ${ }^{1}$ It has been reported that different units of the MicroStar reader provided different rates of loss of the OSL signal per readout using the same stimulation time. ${ }^{74}$

Because the loss of the OSL signal as a function of sequential readouts is related to the OSL curve, the higher rate of loss values of the OSL signal for the high doses reported here is related to the fact that the OSL curves present a faster decay rate in $\mathrm{Al}_{2} \mathrm{O}_{3}: \mathrm{C}$ OSLDs exposed to higher doses. ${ }^{39,41}$

The OSL as a function of stimulation time is characterized by an exponential-like behavior. Thus, the loss of the OSL signal as a function of sequential readouts should also follow an exponential-like behavior. However, the stimulation intensity in the weak- 
stimulation mode is sufficiently weak so that one can assume that the loss of the OSL signal is linear over 100 sequential readouts. On the other hand, in the strong-stimulation mode, one cannot make this assumption. Therefore, we used an exponential model to determine the rate of loss of the OSL signal for the strong-stimulation mode. ${ }^{1}$

\subsection{Fading of OSL signal}

The fading data observed for the two detectors exposed to 10 Gy have very different halflife values [see Table 4-3]. This may be attributed to different room temperatures, up to $\pm 2^{\circ} \mathrm{C}$, during the readouts and/or irradiations of the detectors. We should also mention that the fading data could have systematic uncertainties, which were not taken into consideration, including inaccuracies in the time elapsed since irradiation and readout sessions. ${ }^{1}$

The fading effect has been studied and previously reported in $\mathrm{Al}_{2} \mathrm{O}_{3}: \mathrm{C}$ OSLDs, ${ }^{24,33,34,36,46}$ however these studies did not report a dependence of fading on dose. Jursinic, 2007 reported half-life values in the interval from 0.48 to $0.92 \mathrm{~min}$ using a fitting function slightly different ( $B=0$ in Eq. 3.3) than the one we used in this work [see Eq. (3.3)] and for detectors exposed to doses of $1 \mathrm{~Gy} .{ }^{24}$ We obtained half-life values in the interval from $1.13-2.26$ min by fitting our data to the function used by Jursinic, 2007. The discrepancies in the half-life values may be attributed to different room temperatures during readout, and to the different batches of the sensitive volume of the nanoDot OSLDs. Using the same fitting function as in Eq. (3.3), Mrčela et al., 2011 reported a value of $(1.03 \pm 0.05) \mathrm{min}$, which is consistent with the values reported in this

work. ${ }^{33}$ The study of Mrčela et al., 2011 did not specify the doses to which the detectors 
were exposed. ${ }^{1}$

For 1 and 10 Gy doses, our data showed that at room temperature the OSL signal stabilizes after about 10 min followed irradiation. This is in agreement with the recommendation that nanoDot OSLDs should be read out at least after 8 min delay time

post-irradiation to account for fading of the OSL signal. ${ }^{24}$ The fading effect in the OSL of $\mathrm{Al}_{2} \mathrm{O}_{3}: \mathrm{C}$ can be attributed to the thermal instability of shallow traps at room temperature. $^{64}$

\subsection{Sensitivity changes of nanoDot OSLDs}

NanoDot OSLDs received from Landauer Inc. that had never been used or irradiated produced PMT counts averaging between 550-650 counts in the strong-stimulation readout mode. Typical PMT counts after 1 and 10 Gy were about 70,000 and 790,000 in the weak-stimulation readout mode of the MicroStar reader, respectively. Bleaching the irradiated OSLDs for 600 and $2000 \mathrm{~min}$ in modes (i) and (ii), respectively, was sufficient to produce about 275 PMT counts for $1 \mathrm{~Gy}$ and about 1200 PMT counts for $10 \mathrm{~Gy}$. Note that for long bleaching periods (600 min and $2000 \mathrm{~min}$ for bleaching modes (i) and (ii), respectively) the OSLDs exposed to $1 \mathrm{~Gy}$ presented lower PMT counts than the unirradiated OSLDs. This may be due to background dose received by the un-irradiated OSLDs during transportation and storage because the un-irradiated OSLDs were not bleached before readouts. Note also that we were unable to bleach the OSLDs exposed to $10 \mathrm{~Gy}$ to obtain counts in the background level of the PMT. Therefore, we were unable to completely remove the OSL signal from high-dose irradiations. ${ }^{1}$ 
Figure 4-5a and Figure 4-5d showed a large increase in the residual signals as a function of accumulated dose for the 10 and 55 min bleaching times using modes (i) and (ii), respectively, suggesting that these treatments were insufficient to empty the traps during the bleaching process. The increase in the sensitivity of the OSLDs as a function of accumulated dose, as seen in Figure 4-4a and Figure 4-4d, could be attributed to the high residual levels of the OSL signal as observed in Figure 4-5.'

The residual OSL signals shown in Figure 4-5b, $c$, e and $f$ are not synonymous with the trends seen in the respective Figure $4-4 b, c$, e and $f$ where the nanoDot OSLDs bleached in mode (ii) showed no significant increase in response as a function of postirradiation readout. These observations also contradict the findings from Umisedo et al. $2010,{ }^{72}$ which shows that bleaching wavelengths shorter than $495 \mathrm{~nm}$ cause phototransfer from deep electron traps to the dosimetric trap. Thus, higher residual signal levels should be observed for our OSLDs bleached in mode (i). ${ }^{1}$ For the time being, such trends are unexplained. Further investigations using computer simulations of the charge transport and OSL processes in $\mathrm{Al}_{2} \mathrm{O}_{3}: \mathrm{C}$ may help explain these unexpected trends. This would be done using mathematical software to solve the set of rate equations for the charge transport processes as described in Section 2.2, depending on the choice of $\mathrm{Al}_{2} \mathrm{O}_{3}: \mathrm{C}$ OSL band model. This could provide a phenomenological explanation for our experimental observations.

Bleaching times higher than 120 and $600 \mathrm{~min}$, do not cause a significant sensitivity change in the nanoDot OSLDs exposed to 1 Gy [Figure 4-5b and Figure 4-5c], as there is only a small change of sensitivity of less than $3 \%$, which is within the experimental uncertainty. The sensitivity changes seen in the nanoDot OSLDs exposed to 
10 Gy dose fractions led to a conclusion that the response is highly dependent on the bleaching mode and accumulated dose. Treatment of the detectors with bleaching mode (ii) for at least $600 \mathrm{~min}$ in our bleaching unit is recommended because this mode minimizes changes in sensitivity as a function of accumulated dose for both 1 and $10 \mathrm{~Gy}$ fractions. ${ }^{1}$

For bleaching mode (ii), the residual OSL signals obtained using a bleaching time of 600 min was about four times higher than the residual OSL signals obtained using a bleaching time of $2000 \mathrm{~min}$ for both 1 and $10 \mathrm{~Gy}$ fractions [Figure 4-5b, c, e and f]. However, as observed in Figure 4-4b, c, e and $f$, the sensitivity of the OSLDs remained unchanged, within the recommended 5\% accuracy. For dose fractions of 1 and 10 Gy and total accumulated doses of 7 and $70 \mathrm{~Gy}$, respectively, the level of residual OSL signal of about 10 and 50 times the background level did not affect the sensitivity of nanoDot OSLDs bleached using bleaching mode (ii). Thus, bleaching for more than 600 min using bleaching mode (ii) in our bleaching unit did not provide any additional advantage for the 1 and 10 Gy fractions and accumulated doses investigated in this study. ${ }^{1}$

As was observed in the dose response curves in Figure 4-1, the nanoDot OSLDs presented a linear response until $2 \mathrm{~Gy}$ and a supra-linear response $2 \mathrm{~Gy}$ and above. Thus, the two experiments we performed to investigate the changes in sensitivity as a function of accumulated dose using fractions of 1 and 10 Gy may not be directly comparable because after each cycle of irradiation, those two doses populate the trapping centers in different ways. Further studies are needed to understand the effect of the dose fraction on the sensitivity of nanoDot OSLDs. ${ }^{1}$ 
Sensitivity changes as function of bleaching wavelength, and accumulated dose and stimulation wavelength have been reported in previous studies. ${ }^{24,27,33,36,70} \mathrm{Jursinic}$, 2007 reported a $4 \%$ decrease per 10 Gy accumulated dose in the sensitivity of OSLDs exposed to $1 \mathrm{~Gy}$ dose fractions, after an accumulating $20 \mathrm{~Gy}$ dose. ${ }^{24}$ The same author also reported a decrease in sensitivity in two nanoDot OSLDs exposed to $10 \mathrm{~Gy}$ fractions, for accumulated doses of 60 and $100 \mathrm{~Gy}$, when bleached with $14 \mathrm{~W}$ fluorescent and 150 W tungsten-halogen lamps, respectively. ${ }^{70}$ The author suggested that the change in sensitivity occurs at a supra-linearity threshold of $60 \mathrm{~Gy}$, due to saturation of deep hole traps in the crystal lattice, leading to an eventual increase in the deep electron trap concentration. ${ }^{70}$ However, in our study no significant decrease in the sensitivity was observed in the entire set of nanoDot OSLDs exposed to $10 \mathrm{~Gy}$ fractions, for an accumulated dose of $70 \mathrm{~Gy}{ }^{1}$

Mrčela et al., $2011^{33}$ reported a $2.5 \%$ increase in sensitivity of Dot OSLDs exposed to $1 \mathrm{~Gy}$ dose fractions for an accumulated dose of $8 \mathrm{~Gy}$, when bleached between irradiations using a $75 \mathrm{~W}$ halogen lamp with a filter to partially block ultraviolet photons. ${ }^{33}$ This is in agreement with our findings for nanoDot OSLDs exposed to $1 \mathrm{~Gy}$ dose fractions. ${ }^{1}$

The above results suggest that the sensitivity changes of nanoDot OSLDs depend on the bleaching time, wavelength spectrum of the bleaching light source, reader stimulation wavelength as well as accumulated dose. Short bleaching times, which were insufficient to empty the dosimetric trap, caused the sensitivity of the nanoDot OSLDs to increase as a function of accumulated doses. Bleaching mode (i) (illumination with full emission spectrum of fluorescent light bulbs) caused an increase in sensitivity for high- 
accumulated doses. Conversely, bleaching mode (ii) (illumination with wavelengths larger than $495 \mathrm{~nm}$ of fluorescent light bulbs) led to only a slight decrease in sensitivity for accumulated doses of up to 7 and $70 \mathrm{~Gy}$ (fractions of 1 and $10 \mathrm{~Gy}$, respectively) and bleaching times larger than $600 \mathrm{~min}$. Thus, bleaching mode (ii) for at least $600 \mathrm{~min}$, which utilizes an appropriate long-pass optical filter, is recommended if one wishes to reuse the detectors as it provided the least sensitivity changes. ${ }^{1}$

However, as demonstrated in this work, the change in sensitivity of nanoDot OSLDs as a function of accumulated dose is highly dependent on the bleaching source. Therefore, we strongly recommend that each user properly perform experiments to assess the change in sensitivity as a function of accumulated dose for a specific bleaching source. ${ }^{1}$

\subsection{Calibration protocols}

\subsubsection{Fitting function model}

Our dose response data agreed with the previous dose response experiments (see Section 5.1) and showed a linear response until $2 \mathrm{~Gy} .{ }^{1}$ For doses of $2 \mathrm{~Gy}$ and above, the response was supralinear until the maximum investigated dose of $10 \mathrm{~Gy}{ }^{1}$ Hence, a second [Eq. (3.5)] or third-order polynomial function [Eq. (3.6)] was used to calibrate the dose response data. Since radiotherapy treatments sometimes require dose fractions as high as $10 \mathrm{~Gy}$, non-linear models have to be used to calibrate the dose response.

Other studies ${ }^{24,33,49,60,70}$ have also used non-linear models to fit the dose response curves. Jursinic, $2010^{70}$ used both a second-order polynomial and an exponential model for calibration of nanoDot OSLDs, while Mrčela et al., $2011^{33}$ used a second-order 
polynomial for calibration of Dot OSLDs exposed to doses within the range investigated in this study.

The comparison of both fitting models, using the Fisher's F-test (see Table 4-1) shows that the second-order polynomial function provided the best fit for calibrating the OSLDs. However, using the values of the chi-square relationships, $\chi^{2}$ and $\Gamma^{2}$ [Eq. (3.9) and (3.10)], we determined that Fit 2 - Eq. (3.8) - produced the best absorbed-dose estimations $( \pm 4 \%)$ for all dose fractions, except at 0.2 Gy dose fractions where estimations of the delivered dose differed by up to $\pm 5.8 \%$, depending on the calibration method and bleaching mode. Jursinic $2010^{70}$ reported overestimations of up to $2.5 \%$ below $10 \mathrm{~Gy}$, between the determined dose and delivered dose, for nanoDot OSLDs in a dose range of $0.2-10 \mathrm{~Gy}$, using a second-order polynomial model as in Fit 1 and bleaching for $180 \mathrm{~min}$ with a $14 \mathrm{~W}$ fluorescent lamp. However, our results showed underestimations of up to $4 \%$ for Fit 1, while Fit 2 produced no significant underestimations using bleaching mode (i) for $600 \mathrm{~min}$ (with a $26 \mathrm{~W}$ fluorescent lamp).

The discrepancies between Jursinic 2010 and our results could be due to the different bleaching times and wavelengths used in the experiments. This result vindicates our recommendation that each user should properly perform experiments to assess the change in sensitivity as a function of accumulated dose for a specific bleaching source.' Further investigation using bleaching sources of different wavelengths and intensity would be required to validate this recommendation. 


\subsubsection{Bleaching mode}

For the batch calibration method, the changes in absorbed-dose estimates using Fit 2 were within $\pm 3.5 \%$ except at 50 Gy accumulated dose where up to $6.5 \%$ change was obtained for bleaching mode (ii). However, bleaching mode (i) produced smaller $\chi^{2}$ and $\Gamma^{2}$ values relative to bleaching mode (ii) [see Table 4-5].

For the individual calibration method, bleaching mode (i) produced the best absorbed-dose estimations ( $\pm 3.5 \%)$ across all dose fractions, while bleaching mode (ii) produced up to $6 \%$ underestimation in the absorbed-dose at $60 \mathrm{~Gy}$ accumulated dose. The $\chi^{2}$ and $\Gamma^{2}$ values from Table 4-8 were all lower for bleaching mode (i), compared to bleaching mode (ii), except at 2 Gy fractions.

For the reference dose calibration method, bleaching mode (ii) produced slightly lower $\chi^{2}$ and $\Gamma^{2}$ values, except at $10 \mathrm{~Gy}$ fractions [see Table 4-9]. The large differences in the absorbed-dose estimations using this method have been discussed in Section 4.5.5. The need for scaling factors to convert readings of nanoDot OSLDs between the two stimulation readout modes of the reader leads to inaccurate estimations of the absorbeddose. However, it should be emphasized that this drawback is not a deficiency in this calibration method, but a result of our choice of the reference dose in our methodology. Using a reference dose higher than $20 \mathrm{cGy}$, would produce readings in the weak stimulation mode of the reader, eliminating the need for scaling factors at the high accumulated doses.

In light of the above findings, bleaching mode (i) produces the best absorbed-dose estimations for nanoDot OSLDs across all calibration methods investigated. Since bleaching mode (ii) requires a much longer bleaching time of $2400 \mathrm{~min}$, compared to 600 
min for bleaching mode (i), we recommend that nanoDot OSLDs should be bleached with mode (i) for accuracies in absorbed-dose estimates within $3.5 \%$.

\subsubsection{Absorbed-dose determination}

We have determined that the solution to the third-order polynomial model described by Eq. (3.8) provided the best absorbed-dose determination (within $\pm 3.5 \%$ ) for the nanoDot OSLDs across all dose fractions, using bleaching mode (i), except at $0.2 \mathrm{~Gy}$ and $10 \mathrm{~Gy}$ dose fractions. However, the individual calibration method [see Table 4-8 and Figure 4-22] produced the best absorbed-dose determination (within $\pm 4 \%$ ), across the entire dose fractions and highest accumulated dose investigated in this study. The individual calibration method also provided the smallest $\chi^{2}$ and $\Gamma^{2}$ values, when compared to the other calibration methods, after bleaching with mode (i) and using Fit 2 for the absorbeddose determination.

The reference dose calibration method (Figure 4-13) produced significantly larger differences in absorbed-dose estimations (up to $10 \%$ higher than the delivered doses) at higher accumulated doses for $10 \mathrm{~Gy}$ dose fractions. These high values of absorbed-dose estimations are mainly a result of the switch in the stimulation readout modes of the reader. Recalling from Section 4.4, a scaling factor is required to convert the calibration between these two readout modes. Hence, to compare the results from different stimulation modes, we multiplied the data acquired using the weak-stimulation mode by a scaling factor. This scaling factor was used to qualitatively compare results from the two stimulation modes. However, we observed that the sensitivity of nanoDot OSLDs exposed to the $20 \mathrm{cGy}$ reference dose decreases after accumulating high doses at $10 \mathrm{~Gy}$ 
fractions, such that lower OSL signals are produced, switching the readout mode from weak- to strong-stimulation readout intensities, hence the higher response.

For the batch calibration method, the absorbed-dose estimations were within $\pm 5.5 \%$ at higher accumulated doses above $50 \mathrm{~Gy}$. NanoDot OSLDs in the same batch may have slight variations in mass and defect concentrations; hence, the sensitivity of each dosimeter may be slightly different. The normalization of the readout of each nanoDot OSLD by its individual sensitivity factor, as was done in the individual calibration method, produced better absorbed-dose estimation ( $\pm 4 \%)$ across all cycles and dose fractions regardless of the fitting function.

As we have observed in Section 4.4, the sensitivity of nanoDot OSLDs as a function of accumulated dose depends on the bleaching wavelength. ${ }^{1}$ The reason for an overestimation or underestimation of the absorbed-dose at large accumulated doses could be attributed to the fact that sensitivity increases or decreases as a function of accumulated dose, depending on the bleaching intensity and wavelength. Further studies using computer simulations and phenomenological models, describing OSL processes to explain the sensitivity response as a function of bleaching wavelength and intensity are required. Further experiments could also be done using additional bleaching light sources with different wavelengths.

\subsection{Regeneration of OSL signals}

The regeneration of the OSL signal in dark after pre-exposure to a very high dose of 1 kGy was 4 times more pronounced in nanoDot OSLDs bleached without filter (mode i), compared to nanoDot OSLDs bleached with filter (mode ii). Similar rise time values of 
$560 \mathrm{~h}$ and $564 \mathrm{~h}$ were observed for bleaching modes (i) and (ii), respectively. However, the second experiment in which we measured the regeneration of the signals showed different rise times of $956 \mathrm{~h}$ and $424 \mathrm{~h}$, respectively.

The reason for the different regeneration rates as a function of the bleaching mode is unclear to us. The use of this approach is quite impractical, as it requires an extra correction factor accounting for the regeneration, which is inconsistent and leads to inaccuracies. Hence, we do not recommend its use in calibrating the OSLDs.

\subsection{Absorbed-dose determination in OSLDs pre-exposed to $1 \mathrm{kGy}$}

NanoDot OSLDs pre-exposed to $1 \mathrm{kGy}$ and bleached without filter (mode i) [see Figure 4-19 and Figure 4-20] showed differences of up to $\pm 4 \%$ in absorbed-dose determinations, across all dose fractions for the batch and individual calibration methods using Fit 2; while OSLDs bleached with filter (mode ii) showed significant differences of up to $25 \%$ in absorbed-dose estimations.

The reason for the high absorbed-dose determined for bleaching mode (ii) is mainly a result of the scaling factors required to convert OSL signals when readings were performed using the two different stimulation modes of the reader. As we discussed earlier, the need for scaling factors leads to drawbacks in our methodology, affecting the accuracy of the dose determinations. Another drawback in this methodology is the regeneration of the OSL signals, which were not accounted for in our analysis. Because of the regeneration, the OSL signals and the absorbed-doses depend on the storage durations after every bleaching and irradiation cycle. However, we did not maintain a consistent time interval for readouts of the OSLDs pre-exposed to high doses, which may 
lead to different regeneration levels and therefore, discrepancies in the estimations of doses. As a result of the complications introduced by the regeneration of the OSL signal, we discontinued further investigation on the absorbed-dose determination in nanoDot OSLDs pre-exposed to $1 \mathrm{kGy}$.

For practical implications, the time and duration between bleaching and irradiation of the nanoDot OSLDs have to be pre-determined and kept constant for both bleaching modes, with the rates of regeneration accounted for. In ideal clinical situations, this would require much more effort, compared to the other calibration protocols. Hence, this protocol is not feasible for calibration of nanoDot OSLDs for dosimetry.

However, the trend from the results produced by the pre-exposed OSLDs bleached without filter (mode i), shows that the pre-dose calibration method is feasible as the batch calibration method produced absorbed-dose estimations within $\pm 4 \%$ across all dose fractions [see Figure 4-19].

\subsection{Supralinearity response per cycle}

The trend in supralinearity exhibited by nanoDot OSLDs exposed to a $1 \mathrm{kGy}$ pre-dose (Figure 4-25) contradicts the response exhibited by unsaturated nanoDot OSLDs, where the supralinearity increased up to $25 \%$ and $16 \%$, for bleaching modes (i) and (ii), respectively. For a dose of $10 \mathrm{~Gy}$, the supralinearity for the pre-exposed nanoDot OSLDs were only $3 \%$ and $58 \%$ for bleaching modes (i) and (ii), respectively. Unsaturated detectors presented supralinearity of $20 \%$ and $15 \%$ for a dose of $10 \mathrm{~Gy}$, using bleaching mode (i) and (ii), respectively. Therefore, pre-exposure to a high dose decreases or increases the supralinearity of the dosimeters, depending on the bleaching mode. 
The supralinearity responses highlight the complex processes in the lattice of the OSLDs, which at this point we cannot explain. Further studies using phenomenological models describing the OSL processes are needed to understand the response of the OSLDs. 


\section{Summary, Conclusions and Future Work}

\subsection{Summary}

The goal of the project was to determine the best suitable multiple-use calibration protocol for nanoDot OSLDs in the clinic for radiotherapy dosimetry. We have characterized the response to different influence factors and investigated the effects of different bleaching wavelengths on the luminescence response of nanoDot OSLDs. We also investigated four calibration protocols, and determined the applicability of these protocols for multiple use of the nanoDot OSLDs, after exposures to a $6 \mathrm{MV}$ photon linac beam. Below is a summary of our observations:

I. The dose response of nanoDot OSLDs show a linear response until $2 \mathrm{~Gy}$, with a supralinear response afterwards for the dose range investigated $(0-10 \mathrm{~Gy})$. $^{1}$

II. The loss of the OSL signal after successive readouts is dependent on dose. The rate of loss is $(0.287 \pm 0.003) \%$ for the 'strong-' stimulation mode of the reader; and $(0.019 \pm 0.002) \%$ to $(0.031 \pm 0.001) \%$ for the 'weak-' stimulation mode. ${ }^{1}$ The dose threshold for the two readout modes depends on the reader's and dosimeter's sensitivity.

III. The fading of the OSL signal immediately after irradiation is dependent on dose for the first 10 min post-irradiation, with stability in signal afterwards. Fading was more pronounced for OSLDs exposed to $1 \mathrm{~Gy}$, when compared to $10 \mathrm{~Gy} .^{1}$

IV. The sensitivity changes in nanoDot OSLDs exposed to 1 Gy and 10 Gy dose fractions after repeated cycles of irradiation-readout-bleaching-readout, showed that bleaching directly for $600 \mathrm{~min}$ with $26 \mathrm{~W}$ fluorescent lamps to very low residual signals, produced only a $2.3 \%$ sensitivity change for $1 \mathrm{~Gy}$ dose fractions 
and $10 \%$ change for 10 Gy dose fractions; while bleaching for 2000 min with a long pass filter that blocks wavelengths below $495 \mathrm{~nm}$, to equivalent residual signals, produced only a $1.8 \%$ and a $-2.5 \%$ change for 1 and 10 Gy dose fractions, respectively. $^{1}$

V. The dose response data for the nanoDot OSLDs can be fit to second- and thirdorder polynomial models [Eq. (3.7) and (3.8)]. These models can be used for the absorbed-dose determination of unknown doses for the OSLDs. However, the best absorbed-dose estimations were obtained when a third-order polynomial model [Eq. (3.8)] was used as a model for the calibration curve, except at 0.2 Gy dose fractions.

VI. The absorbed-doses determined using the batch and individual calibration methods produced doses within $\pm 4 \%$ of the delivered doses, using the third-order polynomial model [Eq. (3.8)] for dose calculation, across all cycles and dose fractions, except at $10 \mathrm{~Gy}$ dose fractions where the agreement was within $5 \%$ after 50 Gy accumulated dose. The reference dose calibration produced up to $18 \%$ differences in the absorbed-doses after an accumulating dose of $50 \mathrm{~Gy}$, mainly as result of a switch in the stimulation readout mode of the MicroStar reader.

VII. The nanoDot OSLDs pre-exposed to $1 \mathrm{kGy}$ exhibited a regeneration of the OSL signals after bleaching to low residual signals and storage in dark. The rate of regeneration of the OSL signals was dependent on the bleaching mode employed. NanoDot OSLDs bleached without filter (mode i) regenerated four times faster than those bleached with the long pass filter (mode ii) in the first $24 \mathrm{~h}$ of storage. 
VIII. The dose response plots per irradiation-readout-bleaching-readout cycles for the pre-exposed nanoDot OSLDs showed that nanoDot OSLDs bleached without filter (mode i), exhibited a small supralinearity increase of $3-4 \%$ across three cycles while reaching saturation at $5 \mathrm{~Gy}$, while nanoDot OSLDs bleached with filter (mode ii) exhibited an increasing supralinearity up to $58 \%$ across all three cycles investigated.

IX. The pre-exposed nanoDot OSLDs bleached without the filter (mode i) estimated absorbed-doses with an agreement within $\pm 4 \%$ to the delivered doses, using the third-order polynomial model [Eq. (3.8)] for the absorbed-dose calculation, for all three cycles and dose fractions regardless of the calibration method. The preexposed nanoDot OSLDs bleached with filter showed significantly higher changes.

\subsection{Conclusions}

The conclusions reached from the observations are stated below:

I. NanoDot OSLDs should be read $10 \mathrm{~min}$ after irradiation to avoid the room temperature fading effects on the OSL signal. ${ }^{1}$

II. NanoDot OSLDs can be read successively without a significant loss of the OSL signal if the readout is performed using the weak stimulation mode of the MicroStar reader. For readouts using the strong stimulation mode of the reader correction for signal loss needs to be determined for successive readouts of OSLDs. ${ }^{1}$ 
III. We verified that the dose-response of nanoDot OSLDs, using the MicroStar reader, presents a supra-linear response for doses beyond $2 \mathrm{~Gy}$. The nanoDot OSLDs' dose response can be calibrated using a secondorder or a third-order polynomial model.

IV. NanoDot OSLDs can be used in a multiple-use protocol within $\pm 5 \%$ accuracies up to the maximum accumulated doses investigated in this study, which was $70 \mathrm{~Gy}$ for dose fractions of $10 \mathrm{~Gy}$, after bleaching with a light source that has a very small component of photons with wavelengths below about $495 \mathrm{~nm}^{1}$

V. Changes in sensitivity of nanoDot OSLDs depend on the bleaching time, accumulated doses and the wavelength spectrum of the bleaching source employed.

VI. For accuracies within $4.0 \pm 2.1 \%$ in a multiple use protocol for clinical dosimetry in radiotherapy applications, nanoDot OSLDs can be calibrated using the batch and individual calibration methods, with a third-order polynomial function to model and determine the absorbed-dose, for accumulated doses less than 50 Gy. However, the individual calibration method can be used for accuracies within $3.5 \pm 2.1 \%$ over the largest accumulated dose of $60 \mathrm{~Gy}$, used in this study, after bleaching the nanoDot OSLDs directly under the $26 \mathrm{~W}$ fluorescent lamps.

\subsection{Future Work}

This work has described and characterized the response of nanoDot OSLDs to various influence factors including absorbed and accumulated dose, fading of the OSL signal at 
room temperature, loss of the OSL signal to successive readouts, the dependence of the luminescence on different bleaching wavelengths, and calibration protocols for multipleuse of the dosimeter for clinical radiotherapy dosimetry.

However, the processes described and highlighted need to be explained and better understood using computer simulations to model the response of the OSLD. The charges' migration in the lattice of the crystal and processes that lead to OSL emission may be modeled using phenomenological models that describe the OSL charge transport processes as described briefly in Section 2.2. Preliminary studies have been performed using computer simulations to model and describe the OSL processes and preliminary results have been produced, but were not presented in this report.

Besides, the results reported in this thesis were only experimental. Hence, it will be important to test the applicability and validity of the results under actual clinical situations. The nanoDot OSLDs need to be tested for patient in-vivo dosimetry, by performing clinical trials for different radiotherapy treatments and applications.

The nanoDot OSLDs may also be investigated for dosimetry of diagnostic procedures. 


\section{References}

1. Omotayo AA, Cygler JE, Sawakuchi GO. The effect of different bleaching wavelengths on the sensitivity of $\mathrm{Al}_{2} \mathrm{O}_{3}: \mathrm{C}$ optically stimulated luminescence detectors (OSLDs) exposed to 6 MV photon beams. Med. Phys. 2012;39:5457-5468.

2. IAEA. Absorbed dose determination in external beam radiotherapy: An international code of practice for dosimetry based on standards of absorbed dose to water. Vienna: International Atomic Energy Agency; 2000.

3. Izewska J, Andreo P. The IAEA/WHO TLD postal programme for radiotherapy hospitals. Radiot. Onc.:ESTRO. 2000;54:65-72.

4. Essers M, Mijnheer BJ. In vivo dosimetry during external photon beam radiotherapy. Int. J. Radiat. Onc. Biol. Phys. 1999;43:245-259.

5. ICRP. Prevention of Accidental Exposures to Patients Undergoing Radiation Therapy. International Commission on Radiological Protection. 2000;30.

6. Kutcher GJ, Coia L, Gillin M, et al. Comprehensive QA for radiation oncology: report of AAPM Radiation Therapy Committee Task Group 40. Med. Phys. 1994;21:581-618.

7. Mijnheer BJ, Battermann JJ, Wambersie A. What degree of accuracy is required and can be achieved in photon and neutron therapy? Radiot. Onc. 1987;8:237-252.

8. Internation Commission on Radiation Units and Measurements. ICRU Report 24: Determination of Absorbed Dose in a Patient Irradiated by Beams of X or Gamma Rays in Radiotherapy Procedures. Bethesda; 1976.

9. Tung CJ, Wang $\mathrm{HC}$, Lo SH, Wu JM, Wang CJ. In vivo dosimetry for external photon treatments of head and neck cancers by diodes and TLDS. Radiat. Prot. Dosim. 2003;111:349-356.

10. Higgins PD, Alaei $P$, Gerbi BJ, Dusenbery KE. In vivo diode dosimetry for routine quality assurance in IMRT. Med. Phys. 2003;30:3118-3123.

11. AAPM Report No. 87. Diode in vivo dosimetry for patients receiving external beam radiation therapy: Report of Task Group 62 of the Radiation Therapy Committee. College Park, MD: Medical Physics Publishing; 2005:1-84.

12. Cherpak A, Studinski RCN, Cygler JE. MOSFET detectors in quality assurance of tomotherapy treatments. Radiother. Oncol. 2008;86:242-250. 
13. Cygler JE, Saoudi A, Perry G, Morash C, Choan E. Feasibility study of using MOSFET detectors for in vivo dosimetry during permanent low-dose-rate prostate implants. Radiother. Oncol. 2006;80:296-301.

14. Niroomand-Rad A, Blackwell CR, Coursey BM, et al. Radiochromic film dosimetry: Recommendations of AAPM Radiation Therapy Committee Task Group 55. Med. Phys. 1998;25:2093-2115.

15. Wilcox EE, Daskalov GM. Evaluation of GAFCHROMIC® EBT film for CyberKnife® dosimetry. Med. Phys. 2007;34:1967.

16. Pai S, Das IJ, Dempsey JF, et al. TG-69: radiographic film for megavoltage beam dosimetry. Med. Phys. 2007;34:2228-2258.

17. Suchowerska N, Davison A, Drew J, Metcalfe P. The validity of using radiographic film for radiotherapy dosimetry. Australas. Phys. Eng. Sci. Med. 1997;20:20-26.

18. Almond PR, Biggs PJ, Coursey BM, et al. AAPM's TG-51 protocol for clinical reference dosimetry of high-energy photon and electron beams. Med. Phys.

1999;26:1847-1870.

19. Svarcer V, Fowler JF, Deeley TJ. Exit doses for lung fields measured by lithium fluoride thermoluminescence. Brit. J. Rad. 1965;38:785-790.

20. Izewska J, Georg D, Bera P, et al. A methodology for TLD postal dosimetry audit of high-energy radiotherapy photon beams in non-reference conditions. Radiother. Oncol. 2007;84:67-74.

21. Izewska J, Hultqvist $M$, Bera $P$. Analysis of uncertainties in the IAEA/WHO TLD postal dose audit system. Radiat. Meas. 2008;43:959-963.

22. Viamonte A, Da Rosa LAR, Buckley LA, Cherpak A, Cygler JE. Radiotherapy dosimetry using a commercial OSL system. Med. Phys. 2008;35:1261-1266.

23. Sharma R, Jursinic P. Use of Optically Stimulated Luminescence Dosimeters, OSLDs, for An Independent Verification of Dose Delivered by IMRT Treatments. In: AAPM 2010; 2010.

24. Jursinic PA. Characterization of optically stimulated luminescent dosimeters, OSLDs, for clinical dosimetric measurements. Med. Phys. 2007;34:4594-4604.

25. Yukihara EG, Ruan C, Gasparian PBR, et al. An optically stimulated luminescence system to measure dose profiles in x-ray computed tomography. Phys. Med. Biol. 2009;54:6337-6352. 
26. Ruan C, Yukihara EG, Clouse WJ, Gasparian PBR, Ahmad S. Determination of multislice computed tomography dose index (CTDI) using optically stimulated luminescence technology. Med. Phys. 2010;37:3560-3568.

27. Yukihara EG, McKeever SWS. Optically stimulated luminescence (OSL) dosimetry in medicine. Phys. Med. Biol. 2008;53:R351-R379.

28. Rodrigues $P$, Trindade A, Peralta L, et al. Application of GEANT4 radiation transport toolkit to dose calculations in anthropomorphic phantoms. Appl. Radiat. Isot.

2004;61:1451-1461.

29. Karzmark CJ, White J, Fowler JF. Lithium fluoride thermoluminescence dosimetry. Phys. Med. Biol. 1964;16:273-286.

30. Akselrod MS, Kortov VS, Kravetsky DJ, Gotlib VI. Highly Sensitive

Thermoluminescent Anion-Defective Alpha- $\mathrm{Al}_{2} \mathrm{O}_{3}: \mathrm{C}$ Single Crystal Detectors. Radiat. Prot. Dosim. 1990;32:15-20.

31. Jursinic PA, Yahnke CJ. In vivo dosimetry with optically stimulated luminescent dosimeters, OSLDs, compared to diodes; the effects of buildup cap thickness and fabrication material. Med. Phys. 2011;38:5432-5440.

32. Bos AJJ. High sensitivity thermoluminescence dosimetry. Nucl. Instr. Meth. Phys. Res. B. 2001;184:3-28.

33. Mrčela I, Bokulić T, Izewska J, et al. Optically stimulated luminescence in vivo dosimetry for radiotherapy: physical characterization and clinical measurements in Co-60 beams. Phys. Med. Biol. 2011;56:6065-6082.

34. Schembri V, Heijmen BJM. Optically stimulated luminescence (OSL) of carbondoped aluminum oxide $\left(\mathrm{Al}_{2} \mathrm{O}_{3}: \mathrm{C}\right)$ for film dosimetry in radiotherapy. Med. Phys. 2007;34:2113-2118.

35. Cygler JE, Yukihara EG. Optically Stimulated Luminescence (OSL) Dosimetry in Radiotherapy. In: Rogers DWO, Cygler JE, eds. AAPM 2009 Summer School. College Park, MD: Medical Physics Publishing; 2009:841-864.

36. Reft CS. The energy dependence and dose response of a commercial optically stimulated luminescent detector for kilovoltage photon, megavoltage photon, and electron, proton, and carbon beams. Med. Phys. 2009;36:1690-1699.

37. Aznar MC, Hemdal B, Medin J, et al. In vivo absorbed dose measurements in mammography using a new real-time luminescence technique. Brit. J. Rad. 2005;78:328334. 
38. Mijnheer BJ. State of the art in vivo dosimetry. Radiat. Prot. Dosim. 2008;131:117122.

39. Yukihara EG, McKeever SWS. Optically Stimulated Luminescence: fundamentals and applications. John Wiley \& Sons Ltd. 2011.

40. Chen R, McKeever SWS. Theory of Thermoluminescence and Related Phenomena. Singapore: World Scientific Publishing Co. Pte. Ltd. 1997:559.

41. Bøtter-Jensen L, McKeever SWS, Wintle AG. Optically stimulated luminescence dosimetry. Amsterdam: Elsevier; 2003:29-54.

42. Antonov-Romanovskii VV, Keirum-Marcus IF, Poroshina MS, Trapeznikova ZA. IR stimulable phosphors. In: Conference of the Academy of Sciences of the USSR on the Peaceful Uses of Atomic Energy. USAEC Report AEC-tr-2435, Moscow, 1955, Session of the Division of Physical and Mathematical Sciences. Moscow: USAEC; 1956:239-250.

43. Braeunlich P, Schaefer D, Scharmann A. A simple model for thermoluminescence and thermally stimulated conductivity of inorganic photoconducting phosphors and experiments pertaining to infrared-stimulated luminescence. In: Proceedings of the First International Conference on Luminescence Dosimetry, Stanford, June 1965. Stanford, CA: USAEC; 1967:57-73.

44. Sanborn EN, Beard EL. Sulfides of strontium, calcium, and magnesium in infra-red stimulated luminescence dosimetry. In: Proceedings of the First International Conference on Luminescence Dosimetry, Stanford, June 1965. Stanford, CA: USAEC; 1967:183-191.

45. Huntley DJ, Godfrey-Smith DI, Thewalt MLW. Optical dating of sediments. Nature. 1985;313:105-107.

46. Yukihara EG, Gasparian PBR, Sawakuchi GO, et al. Medical applications of optically stimulated luminescence dosimeters (OSLDs). Radiat. Meas. 2010;45:658-662.

47. Gaza R, McKeever SWS. A real-time, high-resolution optical fibre dosemeter based on optically stimulated luminescence (OSL) of $\mathrm{KBr}: \mathrm{Eu}$, for potential use during the radiotherapy of cancer. Radiat. Prot. Dosim. 2006;120:14-19.

48. Akselrod, M.S.;Kortov, V.S.;Gorelova EA. Preparation and properties of alpha$\mathrm{Al}_{2} \mathrm{O}_{3}:$ C. Radiat. Prot. Dosim. 1993;47:159-164.

49. Yukihara EG, Mardirossian G, Mirzasadeghi M, Guduru S, Ahmad S. Evaluation of $\mathrm{Al}_{2} \mathrm{O}_{3}: \mathrm{C}$ optically stimulated luminescence (OSL) dosimeters for passive dosimetry of high-energy photon and electron beams in radiotherapy. Med. Phys. 2008;35:260-269. 
50. Markey BG, Colyott LE, Mckeever SWS. Time-resolved optically stimulated luminescence from alpha- $\mathrm{Al}_{2} \mathrm{O}_{3}:$ C. Radiat. Meas. 1995;24:457-463.

51. McKeever SWS, Akselrod MS, Markey BG. Pulsed optically stimulated luminescence dosimetry using alpha- $\mathrm{Al}_{2} \mathrm{O}_{3}$ :C. Radiat, Prot. Dosim. 1996;65:267-272.

52. Kasper K, McKeever SWS. Optically stimulated luminescence dosimetry. Nucl. Instr. Meth. Phys. Res. B. 2001;184:29-54.

53. McKeever SWS, Blair MW, Bulur E, et al. Recent advances in dosimetry using the optically stimulated luminescence of $\mathrm{Al}_{2} \mathrm{O}_{3}:$ C. Radiat. Prot. Dosim. 2004;109:269-276.

54. Kron T. Applications of Thermoluminescence Dosimetry in Medicine. Radiat. Prot. Dosim. 1999;85:333-340.

55. Edmund JM, Andersen CE, Marckmann CJ, et al. CW-OSL measurement protocols using optical fibre $\mathrm{Al}_{2} \mathrm{O}_{3}: \mathrm{C}$ dosemeters. Radiat. Prot. Dosim. 2006;119:368-374.

56. Aznar MC, Andersen CE, Bøtter-Jensen L, et al. Real-time optical-fibre luminescence dosimetry for radiotherapy: physical characteristics and applications in photon beams. Phys. Med. Biol. 2004;49:6065-6082.

57. Marckmann CJ, Andersen CE, Aznar MC, Bøtter-Jensen L. Optical fibre dosemeter systems for clinical applications based on radioluminescence and optically stimulated luminescence from $\mathrm{Al}_{2} \mathrm{O}_{3}$ :C. Radiat. Prot. Dosim. 2006;120:28-32.

58. Polf JC, McKeever SWS, Akselrod MS, Holmstrom S. A real-time, fibre optic dosimetry system using $\mathrm{Al}_{2} \mathrm{O}_{3}$ fibres. Radiat. Prot. Dosim. 2002;100:301-304.

59. Polf JC, Yukihara EG, Akselrod MS, McKeever SWS. Real-time luminescence from $\mathrm{Al}_{2} \mathrm{O}_{3}: \mathrm{C}$ fiber dosimeters. Radiat. Meas. 2004;38:227-240.

60. Yukihara EG, Yoshimura EM, Lindstrom TD, et al. High-precision dosimetry for radiotherapy using the optically stimulated luminescence technique and thin $\mathrm{Al}_{2} \mathrm{O}_{3}: \mathrm{C}$ dosimeters. Phys. Med. Biol. 2005;50:5619-5628.

61. Thwaites DI, Mijnheer BJ, Mills JA. Chapter 12 Quality Assurance of External Beam Radiotherapy. In: Podgorsak EB, ed. Radiation Oncology. Vol 1. Vienna, Austria: IAEA; 2006:407-450.

62. Brahme A, Chavaudra J, Landberg T, et al. Accuracy requirements and quality assurance of external beam therapy with photons and electrons. Anders Bra. Acta Oncologica; 1988.

63. Pagonis V, Chen R, Lawless JL. Nonmonotonic dose dependence of OSL intensity due to competition during irradiation and readout. Radiat. Meas. 2006;41:903-909. 
64. McKeever SWS. Temperature dependence of OSL decay curves: Experimental and theoretical aspects. Radiat. Meas. 1997;27:161-170.

65. McKeever S. OSL sensitivity changes during single aliquot procedures: Computer simulations. Radiat. Meas. 1997;27:75-82.

66. Bøtter-Jensen L. Luminescence techniques: instrumentation and methods. Radiat. Meas. 1997;27:749-768.

67. Rogers DWO. General characteristics of radiation dosimeters. In: Rogers DWO, Cygler JE, eds. AAPM 2009 Summer School. College Park, MD: Medical Physics Publishing; 2009.

68. Kerns JR, Kry SF, Sahoo N. Characteristics of optically stimulated luminescence dosimeters in the spread-out Bragg peak region of clinical proton beams. Med. Phys. 2012;39:1854-63.

69. Yukihara EG, Whitley VH, McKeever SWS, Akselrod AE, Akselrod MS. Effect of high-dose irradiation on the optically stimulated luminescence of $\mathrm{Al}_{2} \mathrm{O}_{3}: C$. Radiat. Meas. 2004;38:317-330.

70. Jursinic PA. Changes in optically stimulated luminescent dosimeter (OSLD) dosimetric characteristics with accumulated dose. Med. Phys. 2010;37:132-140.

71. McKeever SWS, Akselrod MS, Colyott LE, et al. Characterisation of $\mathrm{Al}_{2} \mathrm{O}_{3}$ for use in Thermally and Optically Stimulated Luminescence Dosimetry. Radiat. Prot. Dosim. 1999;84:163-168.

72. Umisedo NK, Yoshimura EM, Gasparian PBR, Yukihara EG. Comparison between blue and green stimulated luminescence of $\mathrm{Al}_{2} \mathrm{O}_{3}:$ C. Radiat. Meas. 2010;45:151-156.

73. Miller SD, Murphy MK. Technical performance of the Luxel $\mathrm{Al}_{2} \mathrm{O}_{3}: \mathrm{C}$ optically stimulated luminescence dosemeter element at radiation oncology and nuclear accident dose levels. Radiat. Prot. Dosim. 2007;123:435-442.

74. Aguirre JF, Alvarez P, Amador C, et al. Optically Stimulated Light Dosimetry: Commissioning of an Optically-Stimulated Luminescence (OSL) System for Remote Dosimetry Audits, the Radiological Physics Center Experience. Med. Phys.

2009;36:2591-2592. 